\title{
Ut+EISPP JOURNAL OF COLLEGE ACADEMIC SUPPORT PROGRAMS
}

\section{FEATURED ARTICLES}

Characteristics of Learning

Frameworks Courses in Texas

Public Community Colleges

First-Year Seminars: A

Comparison of Course

Characteristics and High Impact

Practices at Two-Year Colleges

\section{SPECIAL FEATURED ARTICLE}

Completing College: Focus on the Finish Line

\section{PROMISING PRACTICES}

SI or Peer Tutoring: Is One Really Better Than the Other?

Exploring a Vision for a

Global Literacy and Learning Environment: Addressing the Needs of Economically and Culturally Diverse Student Populations Through the Arts Utilizing Google My Maps in the Classroom

\section{EXPLORATORY PIECES}

Learning Alone and Academic Capital: An Old Sociological Idea in a New Educational Application

Building the Discipline-Specific Classroom: A Pedagogical

Discussion

Freshmen, Teaching, and Tenure: Why Do We Call It the Trenches, Anyway? 


\section{EDITOR}

Michael C. McConnell Doctoral Student Graduate Program in DE Texas State University

\section{ASSISTANT EDITOR}

Cassandra Gonzales Doctoral Student Graduate Program in DE Texas State University

\section{EDITORIAL ADVISORS}

Russ Hodges

Associate Professor Graduate Program in DE Texas State University

Emily Miller Payne Associate Professor Graduate Program in DE Texas State University

\section{EDITORIAL ASSISTANTS}

Ann Marie Cotman Doctoral Student Graduate Program School Improvement Texas State University

Tricia Edgel Doctoral Student Graduate Program in DE Texas State University

Meagan A. Hoff Doctoral Student Graduate Program in DE Texas State University

Christie A. Lawson Doctoral Student Graduate Program in DE Texas State University

Rene' LeBlanc Doctoral Student Graduate Program in DE Texas State University

\section{Yuting Lin}

Doctoral Student Graduate Program in DE Texas State University

$\mathrm{Na} \mathrm{Wu}$

Doctoral Student Graduate Program in DE Texas State University

\section{EDITORIAL REVIEW BOARD}

\author{
Sonya L. Armstrong \\ Director of the Doctoral Program in \\ Developmental Education, \\ Texas State University \\ Tammy J. Bishop \\ Department Chair Academic \\ Foundation, Wayne Community \\ College
}

Marsha Cardenas

Coordinator of Developmental

Math Program,

The University of Texas at El Paso

\section{Ruth Dalrymple}

Associate Vice Chancellor

of Academic Partnerships \&

Initiatives, Alamo College District

Tammy Francis Donaldson

Assistant Professor of Reading

\& Faculty Coordinator of

Developmental Education,

Del Mar College; Past

President, Texas Association for

Developmental Education

Cynthia Ferrell

Vice President of the Texas Success

Center at the Texas Association

of Community Colleges

\section{Rochelle Gregory \\ ESFL Division Chair, \\ North Central Texas College \\ Elizabeth Howell \\ Chair of Mathematics Division, \\ North Central Texas College \\ Rebecca Jackson \\ Professor of English and Director \\ of the MA in Rhetoric and \\ Composition, Texas State University}

\section{Forrest Lane}

Associate Professor,

Sam Houston State University

Denise Lujan

Director of Developmental Math,

The University of Texas at El Paso;

President, National Organization

for Student Success

\section{Nara Martirosyan}

Assistant Professor, Department of

Educational Leadership,

Sam Houston State University

Trey Miller

Principal Researcher,

American Institutes for Research
Jennifer Phillips-Denny

Coordinator of Tutoring, The Write

Site at Texas Woman's University;

Past President of North Texas

Writing Centers Association

Pamela Sawyer

Collin College; Past President, Texas

Chapter of the College Reading

and Literacy Association

Monica Stansberry

Senior Program Specialist, Dallas

County Community College District;

Past President, Texas Association

for Developmental Education

Tasha Vice

Assistant Professor of Reading,

Texas A\&M University-San Antonio

Erika Warnick

Associate Professor of Reading,

South Plains College

Quentin Wright

Special Assistant

to the Chancellor, Lonestar

College System Office

(C)2019 Journal of College Academic Support Programs (J-CASP) is funded, supported, and endorsed through the Texas Association for Developmental Education (TADE), the Texas chapter of the College Reading and Learning Association (TxCRLA), and the Graduate Program in Developmental Education at Texas State University (education.txstate.edu/ci/dev-ed). The J-CASP is managed, administered, and published through The Education Institute at Texas State University (tei.education.txstate.edu). Design by Russell Gibson Content (erinmarissarussell.wordpress.com/russell-gibson-content). Layout by Cortney Akers, Print \& Mail ServicesTexas State University (auxiliaryservices.txstate.edu/printmail). Logo by On A Roll Designs (etsy.com/shop/ onarolldesigns).

Authors retain copyright and grant the journal right of first publication with the work simultaneously licensed under a Creative Commons Attribution License that allows others to share the work with an acknowledgement of the work's authorship and initial publication in this journal.

The J-CASP is an academic journal for developmental education, learning assistance, and student success. The editorial staff will give preference to authors, institutions, and/or topics relevant to the context of developmental education and learning assistance in Texas. Please review our submission guidelines at journals. tdl.org/ jcasp/index.php/jcasp/about/submissions. Please address any questions, comments, concerns, and suggestions to JCASP_Editor@txstate.edu. 


\section{CASP Conference Bridges to Success}

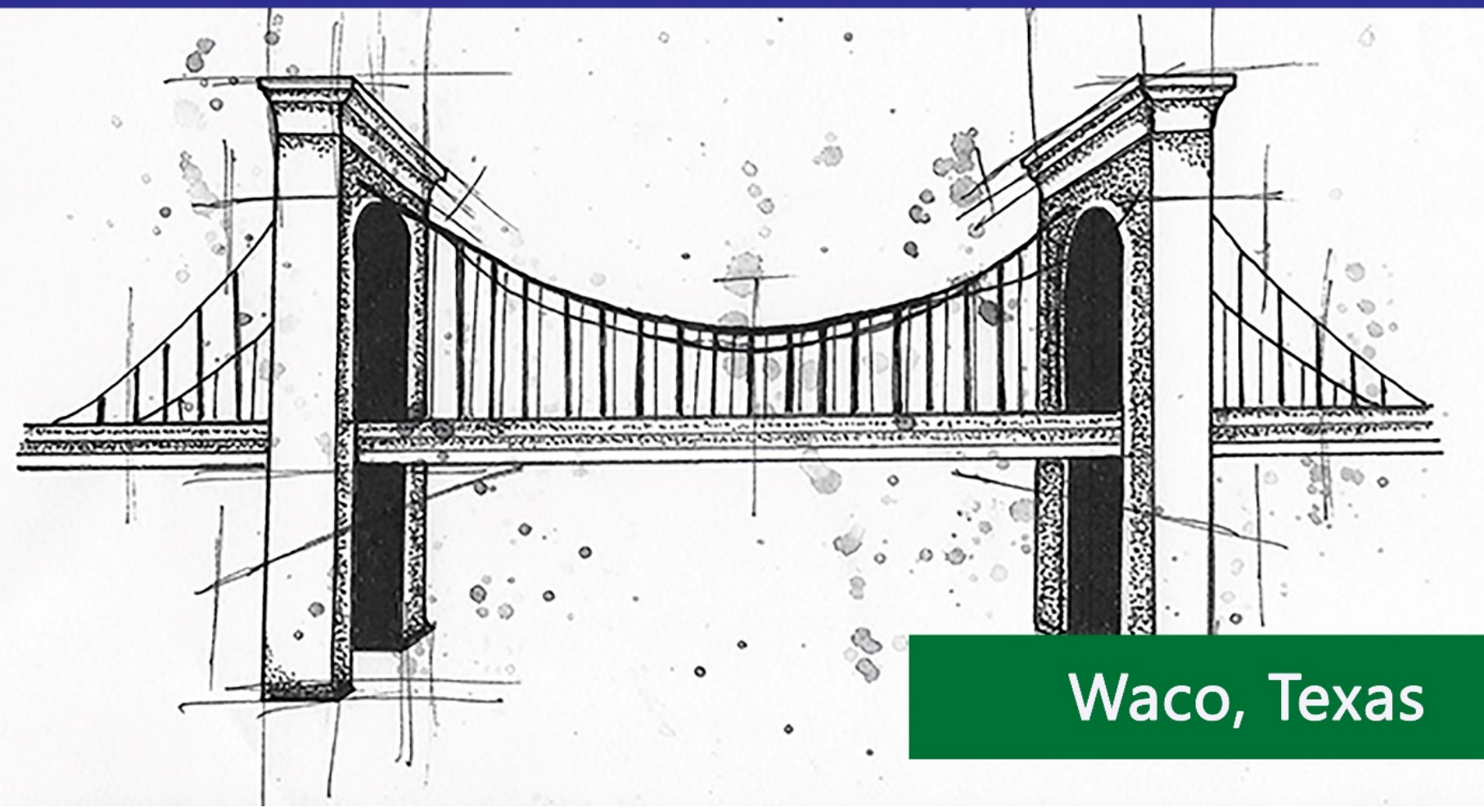

Join us in Waco, Texas

For the 2019 College Academic Support Programs

Conference!

October 20-22, 2019 
CONTENT

5 WELCOME

By Michael C. McConnell, Editor

7 FEATURED ARTICLE

Characteristics of Learning Frameworks Courses in Texas Public Community Colleges

By Russ Hodges

Taylor W. Acee

Stephanie M. Jarrett

René Leblanc

Yuting Lin

Amarilis M. Castillo

Christie Hill-Troglin Cox

Christie Lawson

Candice P. Oelschlegel

Darolyn A. Flaggs

\section{FEATURED ARTICLE}

First-Year Seminars: A Comparison of Course Characteristics and High Impact Practices at Two-Year Colleges

By Forrest C. Lane Andrew P. Miller

36 SPECIAL FEATURED ARTICLE

Completing College: Focus on the Finish Line

By Hunter R. Boylan

Barbara J. Calderwood

Barbara S. Bonham

\section{PROMISING PRACTICE}

SI or Peer Tutoring: Is One Really Better

Than the Other?

By Klara Keim

Michelle Kiser

55 PROMISING PRACTICE

Exploring a Vision for a Global Literacy and Learning Environment: Addressing the Needs of Economically and Culturally Diverse Student Populations

Through the Arts

By Neva Cramer

Joan Bowman
59 PROMISING PRACTICE

Utilizing Google My Maps in the Classroom

By William S. Duffy

62 EXPLORATORY PIECE

Learning Alone and Academic Capital:

An Old Sociological Idea in a New Educational Application

By Jack Trammell

65 EXPLORATORY PIECE

Building the Discipline-Specific Classroom: A Pedagogical Discussion

By Leta Deithloff

68 EXPLORATORY PIECE

Freshmen, Teaching, and Tenure: Why Do We Call It the Trenches, Anyway?

By Ann-Marie Lopez

COVER PHOTO

Old Main and the Eclipse

By Andy Heatwole smtxphotos.com

SECTION BREAK

The Swing

By Christopher Paul Cardoza

SECTION BREAK

51

Post Road

By Karen Johnston-Ashton

SECTION BREAK

6:00 a.m.

By Jacklyn Miller

jacklynmiller.art 


\section{FOREWORD}

Welcome to another issue of the Journal of College Academic Support Programs (J-CASP). The first two issues were highly successful, and according to our website analytics, each issue was viewed and/or downloaded approximately 800 times. We are very grateful for such a reception for the journal's inaugural year as a platform for scholarship in the fields of developmental education (DE), learning support, and student success in Texas.

This issue features a triad of articles representing DE not only in Texas but across the country. Two represent professionals from both colleague DE doctoral programs in Texas, one from Texas State University about learning frameworks, and the other from Sam Houston State University about first-year seminars. The third article-a special feature article-a reprint of a white-paper from the National Center for Developmental Education, addresses reform movements in DE and college completion throughout the nation.

Furthermore, this issue contains multiple non-juried articles that showcase practitioners. Included are three promising practices articles: one about incorporating the arts into literacy instruction, one about utilizing the technology-based platform Google My Maps into classroom projects, and one that discerns supplemental instruction from tutoring and addresses the benefits of either or both in tandem. Also, this issue also showcases three exploratory articles: one introducing academic capital as a further extension of the notion of social capital, one proposing contextualized literacy curriculum, and one exploring first-year student retention and the relegation of important gateway courses to adjunct instructors.

The J-CASP staff is proud to announce and clarify our new genre-exploratory pieces. The notion rose from a roundtable session of authors, editors, editorial review-board members, and conference participants from the 2018 conference of the College Academic Support Services, which graciously endorses and funds-along with the Graduate Program in Developmental Education at Texas State University-this journal. An exploratory piece puts forth an idea that might deserve further scholarly exploration. As higher education and student success and retention is a continually evolving and changing field, new ideas, strategies, and vision continually abound.

On behalf of the J-CASP editorial staff, thank you for reading this journal. We hope that you enjoy the wealth of scholarship contained in these pages.

Michael C. McConnell, Editor

Journal of College Academic Support Programs 


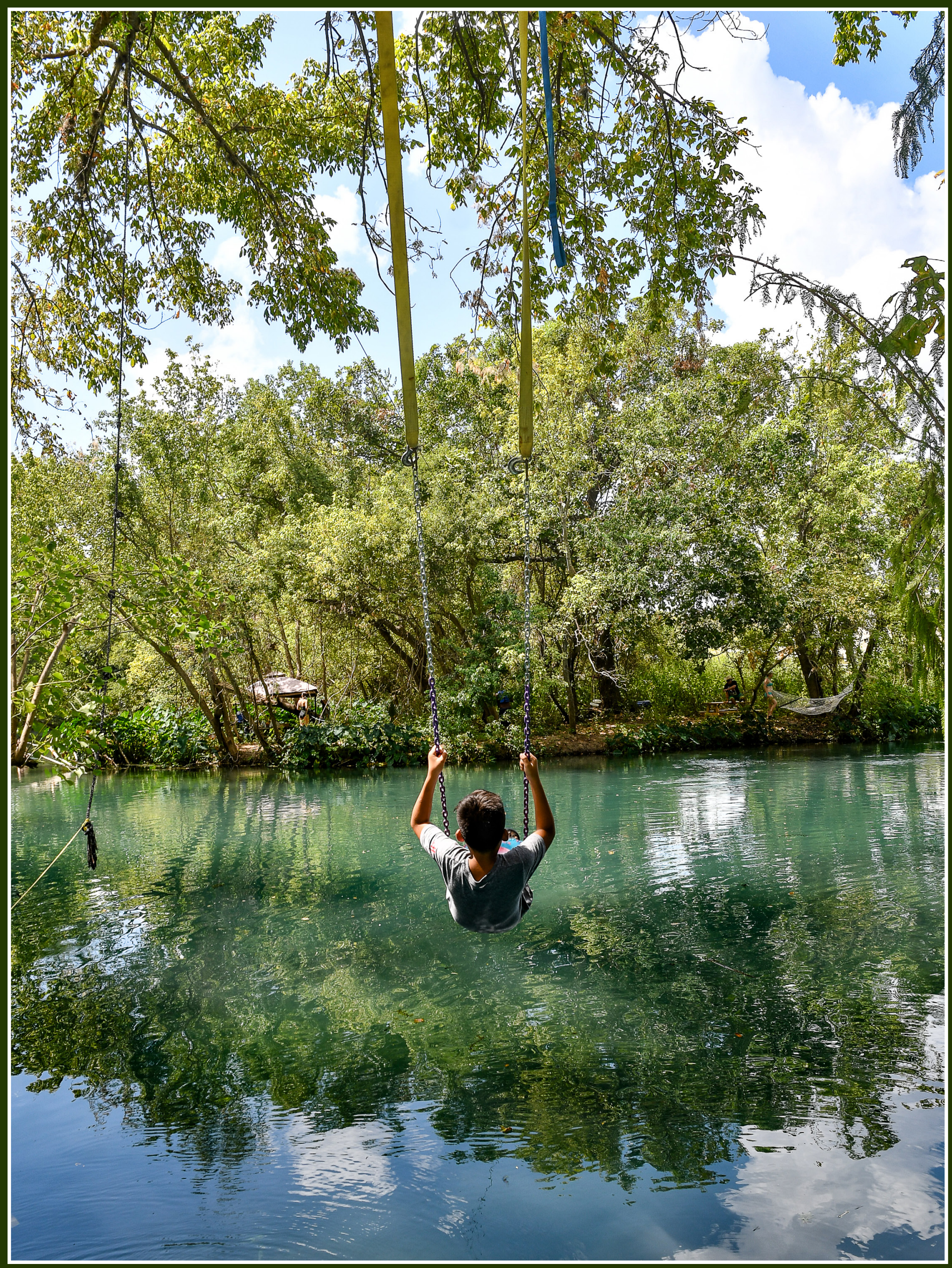




\section{Characteristics of Learning}

\section{Frameworks Courses in Texas Public}

\section{Community Colleges}

\author{
Russ Hodges, Texas State University
}

Taylor W. Acee, Texas State University

Stephanie M. Jarrett, Texas State University

René Leblanc, Texas State University

Yuting Lin, Texas State University

\author{
Amarilis M. Castillo, Texas State University \\ Christie Hill-Troglin Cox, Texas State University \\ Christie Lawson, Texas State University \\ Candice P. Oelschlegel, Texas State University \\ Darolyn A. Flaggs, Kennesaw State University
}

\section{ABSTRACT}

In this qualitative study, the authors extend the previous research and present findings from a study investigating postsecondary theoretically-based study strategy courses. In Texas, these courses are known as learning frameworks courses, offered for college credit, and derive full-formula funding from the Texas Higher Education Coordinating Board. Researchers focused on learning frameworks courses offered at Texas public community colleges in the Fall 2016 and Spring 2017 semesters. A total of 44 course coordinators or their designees were interviewed via phone or email. Using content analysis, researchers coded data into content categories and thematic units. Findings provide historical, administrative and curricula perspectives including primary distinctions among course topics; theoretical perspectives (or lack thereof); textbooks, resources, and assessment selections; and instructor training. The authors recommended the development of statewide resources to assist institutions in meeting statewide curricula requirements.

ne approach to support students' learning in postsecondary education is the offering of learning frameworks courses, also referred to as strategic learning, learning-to-learn, and student success courses-among other names. Offered for college credit in either one-, two-, or three-hour course formats, the hallmark of the curriculum is to introduce students to theories from cognitive, behavioral, and affective domains of educational psychology to underpin the learning strategies taught within the course. The primary goals are fostering students' comprehension of themselves as learners; helping them to increase their self-efficacy, self-regulation and motivation to succeed; teaching them to understand the reasons for engaging in specific study behaviors; and utilizing and transferring new study behaviors to their other courses by embedding the strategies within a disciplinary context. In this paper, we trace the history of learning frameworks courses nationally and within Texas, review research of their effectiveness, and present original findings from a research study investigating the characteristics of learning frameworks courses offered at Texas public community colleges.
Historical Review of Learning Framework Courses

The practice of guiding students in how to succeed in college, including how to navigate the challenges college creates, has its roots in the late 1800s when a new type of course began to appear: first semester courses focused on helping students through the heightened challenges of pursuing higher education (Keup \& Barefoot, 2005). Still offered today, freshmen seminar courses, now typically titled First-Year Seminar are often part of a robust First-Year Experience Program, offered for one-credit hour, and focus on helping students' transition, acclimate, and integrate into the college environment (Agee \& Hodges, 2018).

The Medical Model of Study Skills Instruction

Beginning in the 1920s, courses that focused specifically on study skill development emerged and grew as instructors and students realized the need for them. These courses focused on note-taking, reading comprehension, textbook study methods, time management, examination preparation, and test-taking (Maxwell, 1997). Delineating the sources of textbooks propounding college study skills, Stahl and Henk (1986) listed Whipple's (1927) How to Study 
Effectively and Bird's (1931) Effective Study Habits. Stahl and Henk (1986) also included other such works including the Student's Guide to Effective Study (Cole \& Ferguson, 1935), the How to Study Handbook (Frederick, 1938), and Diagnostic and Remedial Techniques for Effective Study (Robinson, 1941).

Yet, most textbooks addressing study skills did not include theoretical perspectives on learning or studying, nor did they advise that students be introduced to learning theory. Instead, these books' content focused on instructional philosophy based on remediation involving practice and skill. Like the medical model, these authors' goal was helping instructors diagnose students' skill and study deficits and then directing them to lessons designed to address students' areas of weakness. By the middle of the 1940s, more than 100 courses began appearing that addressed how to study for students admitted on academic probation and for students needing remedial help. Robinson advocated using his study method Survey Q3R or, as it is now known, SQ3R to address how to survey, question, read, recite, and review in his second study skills book, Effective Learning (1936). Robinson described SQ3R as a system supported by scientific management and a higher-level way to study (Stahl \& Henk, 1986). Robinson also claimed that his course offered at The Ohio State University addressed how to study effectively for students on- and off-academic probation, and for students that were returning soldiers from World War II. Robinson's (1946) one caveat was that "brighter students benefited the most" from it (p. 1). Through the mid-1980s, these books' authors continued to focus on instructional philosophy based on remediation involving practice and skill. Even when updated in 1974, the seminal textbook How to Study in College (Pauk, 1962) focused on skill domains such as the basic on-going skills, the academic skills, and the supportive skills. Addressing the book's lack of theoretical grounding, Pauk argued, "Students are not primarily interested in theory ... After all, the person who needs penicillin is seldom cured by learning the history of antibiotics" (p. vii, 1974). The same was true for Ellis's bestselling textbook Becoming a Master Student (1985). His book, claimed to be filled with "tools, techniques, hints, ideas, illustrations, instructions, procedures, processes, skills, resources and suggestions for success" (1985, inside cover). Yet, void were theory and research-based citations to support the skills and strategies he promoted.

\section{Emergent Learning Frameworks Courses}

Educators at two Texas institutions were at the forefront of developing emergent learning frameworks courses; their research findings, conducted in the 1990s, began to acknowledge that students learning the theory underlying why study strategies work is essential to grasp holistically, retain, and utilize study strategies. At Texas State University (then Southwest Texas State University), Sellers created a course in 1973 similar to other college reading and study skills courses of the 1970s, with topics such as reading comprehension, vocabulary building, note-taking, time management, and speed reading. However, 1980s theory and research integration slowly transformed the curriculum into an applied learning and behavior management course supported by behavioral, cognitive psychology, adult learning, and student development theories (Hodges, 2014).

At the University of Texas at Austin in 1975, Weinstein also began creating a theory-based learning frameworks course that over time became more rooted in emerging research on learning strategies instruction and disassociated from skill-anddrill study skills instruction (Hodges \& Acee, 2017). Learning strategies have been defined as "behaviors and thoughts in which a learner engages and which are intended to influence the learner's encoding process ... . and affect the way in which the learner selects, acquires, organizes, or integrates new knowledge" (Weinstein \& Mayer, 1983, p. 1). Studies by Weinstein, Dierking, Husman, Roska, and Powdrill (1998) and Hodges, Seller, and Dochen (2001) provided impetus for learning frameworks courses at the state level by showing statistically significant improved retention and graduation rates for students successfully completing these courses as compared to similar students not enrolled.

In 1997, Cole, Babcock, Goetz, and Weinstein introduced the term learning framework[s] as a course fostering students' regulation of learning by developing perspectives of themselves as learners. The intention guiding this course was the idea that increasing students' metacognitive understanding of themselves and how they use learning methods could motivate, foster, and facilitate transfer of learning strategies into courses in which students experienced difficulty.

Prior to 1999, academic success courses could be offered at higher education institutions in Texas, 
but all such courses could not generate formula funding. In October 1999, a proposal was submitted to the the Texas Higher Education Coordinating Board (THECB) by Texas State University to change this funding policy based on research and the increased success of students enrolled in what was termed "learning framework[s]" courses. Based on that proposal, the THECB authorized formula funding (of up to three semester credit hours per student) for learning frameworks courses which must focus on "1) research and theory in the psychology of learning, cognition, and motivation, 2) factors that impact learning, and 3) application of learning strategies" (Hill, 2000, p. 1). The critical characteristic of such a course, according to the THECB, "...is the presence of theoretical models as the curricular core" (Hill, 2000, p 1).

As learning frameworks courses became formally recognized in Texas, researchers across the country continued to explore the effects of underpinning theory to learning strategies. Hofer and $\mathrm{Yu}$ (2003) researched a psychology course emphasizing self-regulation as a core principle integrating teaching cognitive, metacognitive, behavioral, and social cognitive theory with practical learning, self-regulation, and motivational strategies. Hofer and Yu assessed the course's impact by using Pintrich, Smith, Garcia, and McKeachie's (1993) Motivated Strategies for Learning Questionnaire (MSLQ) as a pre- and postcourse assessment of students and found that the average differences and correlational results showed that students' self-efficacy about learning, their focus on mastery goals, and their sense that the course was important increased. The study also showed that text anxiety decreased. In 2004, Dembo and Seli furthered this work on goal orientation and self-efficacy by showing students why they could and should change their study behaivors. The authors employed a four-step process using self-evaluation, goals, learning strategies, and self-assessment and regulation and used results from two self-report surveys to collect data. The final survey targeted students who had previously stressed that they did not want to change their study behavior because they did not think they needed to. Results showed that, on average, students who did not want to change at course onset believed that they had changed positively as a result of the four-step process. These early studies on learning frameworks courses helped to establish their legitimacy in the eyes of researchers and policy makers, and paved the way for scaling up research and practice in this area.

\section{Recent Investigations}

While focusing on psycho-social variables leading to student retention and inclusion in higher education, Kennett and Reed (2009) studied how academic performance and retention could be improved via a theory-to-practice course. They studied a success course that embedded learning and memory theory, motivation, stress-coping skills, and problem solving and included applications for practicing theory and aiding in its generalization. Data findings indicated that students who were more impulsive and had the lowest self-efficacy were aided most in the course.

In 2011, Tuckman and Kennedy conducted a large investigation of their online, hybrid model, learning theory-driven study course. Using a matched control group of 351 students not taking the learning theory-based study course, the researchers measured the outcomes of the first four terms of both groups' college careers. Though retention status declined overall, course-takers maintained a higher retention rate over the four semesters (93.4\%) compared to non-course-takers (85.5\%), and a higher GPA with increased odds of graduation (1.69\% higher than non-course takers). In another study focused on an online theory-based study skills course-Pryjmachuk and Gills (2012) found that students' confidence and knowledge had grown based on qualitative pre and post surveys and interviews conducted by the researchers. Approaching learning theory-based study strategy courses from yet another angle, Urciuoli and Bluestone (2013) linked a student success course to a content-based psychology course and, using the Learning and Study Strategies Inventory (LASSI) as a pre- and post- assessment, found a small effect on attitude (or students' interest regarding college activities and achievement) and concentration. Other investigations by Hoops, $\mathrm{Yu}$, Burridge, and Wolters (2015) and Wolters and Hoops (2015) found that teaching students theory and strategies increased students' self-regulation behaviors.

Also in 2015, Bartoszewski and Gurung used the work of Dunlosky, Rawson, Marsh, Nathan, and Willingham (2013), whose study focused not only on ten specific study strategies and their effectiveness, but also on how these strategies generalized across learning conditions, materials, student traits, and tasks relevant to students' achievement. Of the strategies discussed by Dunlosky et al. (2013), Bartoszewski and Gurung (2015) found that only elaborative interrogation, a form of self-testing involving knowledge retrieval, predicted exam scores in a multiple regression analysis, reinforcing the importance of self-testing as an important learning strategy.

In 2015, Fong, Zientek, Ozel, and Phelps (2015) examined another sparsely researched area related to study strategies: how self-efficacy in motivation, self-regulation, handling of learning resources, and 
the strategies students apply in learning is bound to student ethnicity. Their study of a developmental mathematics course found some significant variations in self-efficacy according to students' ethnicities. This study has broader implications for the increasing diverse populations in colleges and for student retention by changing instruction according to the differences in self-efficacy.

Examining college students' perceptions of a theory-based college success course, Hoops and Artrip (2016) reported that students claimed time management and motivation were most important for being effective. Karp, Raufman, Efthimiou, and Ritze (2017) determined that pedagogies integrating college orientation activities, disciplinary content, and academic success skills resulted in higher grade point averages and more credits. In 2018, Hensley, Wolters, Won, and Brady indicated that time management taught within student success courses supported self-regulation development based on a study conducted with probationary and non-probationary students.

While self-regulation is important for students in study strategies course success, in their study, also from 2018, Howard, Moret, Faulconer, Cannon, and Tomlin argue that study skills courses are most effective when they not only teach study strategies and the concept of self-regulation, but also when these courses emphasize transfer strategies to other courses. Howard et al. facilitated transfer of study strategies by having students write metacognitive reflections explaining their use of strategies in other courses. These metacognitive reflections, according to the researchers, proved essential for transfer to occur.

Researchers have also found that students' metacognitive awareness of why some strategies were more effective than others influenced their use of achievement goals (Geller, Toftness, Armstrong, Carpenter, Manz, Coffman, \& Lamm, 2018). Driven by "metacognitive awareness" (Geller et al., 2018, p. 683), successful students scheduled practice times for self-testing and studying when they realized that they studied more effectively this way. The researchers posited that most students, without instruction, lack knowledge of what works and why, and continue using unproductive methods.

In sum, learning frameworks courses have been found to help support students' development as strategic learners and enabled them reach higher levels of success in college. Since 1999, when the

THECB approved formula funding, the prevalence of learning frameworks courses in Texas public colleges has grown; many community colleges are even requiring these courses of incoming students. However, little is known about the approaches being used in these courses, topics covered, and the extent to which courses vary from institution to institution. Describing the characteristics of learning frameworks courses at Texas public community colleges could help to reveal common themes, innovative practices, and advance both learning frameworks research and practice. Describing the various approaches used in learning frameworks courses could help educators and administrators make more informed decisions about how to structure and implement their courses. This information could also be valuable to scholars interested in studying the effectiveness of learning frameworks courses across different institutions.

\section{frameworks}

courses became

Texas, researchers across the country continued to explore the effects of underpinning theory to learning strategies.

\section{Methods}

The purpose of the current study was to describe characteristics of learning frameworks courses offered at Texas public community colleges in the Fall 2016 and Spring 2017 semesters. The overarching research question was: What are historical, administrative, and curricular characteristics of learning frameworks courses? From a historical perspective, we were interested in when each institution's course was originally established and how it changed over the years. Administrative characteristics of interest included the population of students served, whether it was required or paired with another course, how it was marketed to students, and the types of training provided to instructors. Curricular characteristics of interest were the types of textbooks and assessments used, and the types of topics covered in the course. To obtain data to help answer our research question, we conducted interviews with the community college coordinators (or designees) of learning frameworks courses. We also obtained syllabi for the learning frameworks courses and content analyzed them to determine the types of topics covered.

Institutions with Learning Frameworks Courses

The Texas Higher Education Coordinating Board recognizes 50 public community colleges in Texas. To determine which institutions offered learning frameworks courses in the Fall 2016 and Spring 2017 semesters, we searched each institution's website (e.g., online course catalogs and class schedules) 
for course offerings that used the state's designated learning frameworks course prefix/numbers (i.e., EDUC 1100, 1200, 1300; PSYC 1100, 1200, 1300). Out of the 50 total public community colleges, we found that 45 had a learning frameworks course and five did not (see Appendix A). One institution reported designing a learning frameworks course under a different prefix/number for health science professionals, and it was retained in the study. It should be noted that the institutions without learning frameworks courses also may have had courses that offered similar content under a different prefix/numbers that went undetected. Of the 45 institutions with learning frameworks courses, eight had multiple campuses and 37 had a single campus.

\section{Participants}

We used a variety of methods to determine the coordinator of each learning frameworks course. These methods involved searching institutional websites, querying institutions via email and phone, and using our personal contacts to help locate the correct person to interview. As part of this process, we recognized that some institutions had a central coordinator that oversaw learning frameworks courses across multiple campuses. In this case, we sought to interview a single person to speak on behalf of the learning frameworks courses being implemented on each campus. When the opposite was true, we sought to interview the learning frameworks coordinator from each campus. Of the eight multi-campus institutions, 4 had one coordinator working across multiple campuses, 2 had separate coordinators for each campus, and 2 had an unknown status because we were unable to contact anyone who could provide this information. Ultimately, a total of 49 learning frameworks coordinators were identified and contacted for an interview, of which 44 agreed to be interviewed (three were designees of the coordinator), yielding a $90 \%$ response rate. See Appendix A for a listing of each institution included in this study and details about the learning frameworks course prefix/numbers used coordinator name, and interview method.

\section{Research Design and Data Collection}

The current study was a descriptive study that used qualitative data obtained through semi-structured interviews and content analysis of syllabi. The interviews were conducted with learning frameworks coordinators (or their designees) over the phone ( $n$ $=38$ ) or via email $(n=6)$, when a phone meeting was unable to be scheduled. For phone interviews, trained graduate student researchers asked questions to the interviewee and recorded their responses into a spreadsheet. Phone interviews lasted approximately 45-90 minutes. For email interviews, the interview questions were sent in a Word document for the interviewees to complete and send back via email. A total of 27 questions were included in the interview protocol, in addition to probing questions (for a full listing of these questions contact the senior author). For the purposes of this study, we analyzed data from 10 interview questions (see Appendix B).

In addition, we obtained an example syllabus or detailed syllabus template that represented the learning frameworks courses offered at each interviewee's institution. To determine the types of course topics covered in these courses, we extracted every course topic listed in the course calendar of the syllabus.

\section{Data Analysis}

Using content analysis (Krippendorff, 2004), all recorded data was segmented into smaller, interpretable units of analysis, coded into content categories, and then combined into larger thematic units, when applicable. For interview data, researchers examined interviewee responses that were previously recorded into a spreadsheet. Many of the questions were yes/no questions and probed for examples. The results below report the percentage of yes/no responses for each question and further provide content categories that represent the types of examples given, in some cases we also provide specific examples. Content analysis was also used to examine course topics. An expert researcher/ practitioner in the field of learning frameworks read each course topic, coded it into a content category, and subsequently grouped these content categories into larger themes, when applicable.

\section{Findings}

We organized the findings into historical, administrative, and curricular characteristics of learning frameworks courses. Findings are based on content analyses of interview responses with learning frameworks coordinators (or designees). We also present findings of a content analysis of course topics listed in learning frameworks course calendars obtained from interviewees.

\section{Historical Characteristics}

When the courses began. Interviewees were asked to report when their respective Texas public community college began offering learning frameworks courses. The question presented allowed the participant to respond based on their own knowledge of their institutions. While many of the respondents were clear on when the learning frameworks course began, not all were certain of the exact year. Table 1 shows that most institutions initiated their learning frameworks programs within the last decade. In addition, two respondents indicated that the learning frameworks course had begun initially, ceased at some point, and returned to the institution years later. 
Table 1

\begin{tabular}{lcc}
\multicolumn{2}{l}{ Inception of Each Learning } & Frameworks Course \\
\hline Source & $F$ & $\%$ \\
Prior to 2000 & 2 & 4 \\
$2000-2005$ & 6 & 14 \\
$2006-2010$ & 11 & 25 \\
$2011-2015$ & 22 & 50 \\
Undetermined & 3 & 7 \\
\hline Total & 44 & 100 \\
\hline
\end{tabular}

How the courses changed. Of the 44 respondents, 32 noted changes to the learning frameworks course over the years, nine noted no mentionable changes, and three noted that changes were unclear or not applicable. Interviewees who indicated changes commonly listed multiple changes. Content analysis of the example changes provided by interviewees revealed various administrative and curricular changes with the most common being changes to the curriculum ( $n=14)$, textbook ( $n=12)$, and changes in the number of credit hours awarded $(n=11)$. Additional types of changes were to lessons and activities $(n=7)$, making the learning frameworks course a core requirement $(n=4)$, changes to student learning outcomes $(n=3)$, the governing department ( $n$ $=3)$, assessments $(n=2)$, eligible students $(n=2)$, the course name $(n=1)$, class size $(n=1)$, and more rigorous instructor credentials $(n=1)$. It should be noted that some of the curricular changes demonstrated attempts to make the course more in-depth and theory-based. For example, one interviewee stated: "It's way more in depth. It used to be...more skills-based for certain classes. Now it's more of a course of learning the theories and approaches that they apply to their other classes, not a tutoring class anymore."

\section{Administrative Changes}

Courses designed for special populations. We also asked interviewees if they offered learning frameworks sections designed for special populations or disciplines. Of the 44 interview respondents, 24 (55\%) reported learning frameworks sections designed for special populations, with several interviewees listing multiple special populations served. Examples reflected a wide range of responses that included students enrolled with less than 15 credit hours $(n=5)$, those enrolled in dual-credit $(n=4)$, honors program students $(n=3)$, health science majors $(n=3)$, STEM majors $(n=2)$, nontraditional stu- dents $(n=1)$, students on academic probation $(n=1)$, student athletes ( $n=1)$, teacher education majors ( $n$ $=1$ ), fire science majors $(n=1)$, English composition $(n=1)$, student veterans $(n=1)$, Dream Catcher Puente Program $(n=1)$, students with disabilities ( $n=$ $1)$, TRIO $(n=1)$, first generation students $(n=1)$, engineer majors ( $n=1)$, students enrolled in technical programs such as welding and air conditioning tech $(n=1)$, criminal justice majors $(n=1)$, and cosmetology $(n=1)$.

Courses designed for developmental educa-

tion. We were also interested if any of the learning frameworks sections were specifically designed for students enrolled in developmental education courses. Of the 44 interviewees, eight (18\%) responded that they offered a course designed specifically for students in developmental education courses; of those, six reported the learning frameworks course was required for this student population.

Mandated enrollment. Respondents were further asked if their learning frameworks courses were mandated and, if so, for whom. Of the 44 respondents, $12(27 \%)$ indicated it was mandated for all students, 20 (45\%) reported that it was mandated for some students, $10(23 \%)$ indicated it was not mandated, and 2 said they did not know. When probed to explain who their courses were mandated for, institutions frequently described more than one student population. Of those stating that their courses were mandated for some, the following types of examples were provided: First Time in College (FTIC) students and those with less than $12-15$ credit hours $(n=10)$, based on Texas Success Initiative Assessment (TSIA) scores $(n=9)$, students on suspension and academic probation $(n=5)$, those in a general studies program $(n=2)$, college preparatory program $(n=2)$, Dreamers program $(n=1)$, Burleson Opportunity Fund Scholarship program $(n=1)$, and Mathways program $(n=1)$. Several interviewees mentioned that their institution was in the process of mandating it or still deciding whether to mandate it in future semesters. Also, one interviewee noted that although the course was not officially mandated, advisers commonly describe it to students as a mandatory elective. Finally, it should be noted that $32(72 \%)$ of respondents indicated that their learning frameworks course was mandated for all students or all FTIC students with less than $12-15$ credits.

Pairing of learning frameworks course. Interviewees were asked if they paired their learning frameworks courses with another course. Of the 44 interviewees, 15 (34\%) indicated that their learning frameworks courses were paired with other courses during the semesters in question, whereas 28 (64\%) indicated no pairings and one interviewee was unable 
to say definitively if the courses were paired, because pairing happened through informal arrangements with instructors. Our content analysis of the pairedcourse examples provided by the 15 interviewees (note some interviewees mentioned multiple course pairings) suggested that most were paired with a literacy course: English Composition $(n=7)$, English as a second language $(n=1)$, reading $(n=1)$, integrated reading and writing $(n=3)$. Learning frameworks courses were also paired with mathematics course $(n=6)$. Other learning frameworks course pairing mentioned include: biology $(n=1)$, psychology $(n=$ $1)$, history $(n=1)$, introduction courses $(n=1)$, workforce courses $(n=1)$, and developmental education courses in general $(n=1)$.

\section{Marketing learning frame-}

works courses. Because marketing strategies were expected to be fundamentally different for institutions that mandate their courses compared to those that do not, responses to the question about how the learning frameworks courses are marketed or advertised were divided into two categories: (a) marketing of learning frameworks that were mandated and (b) marketing of learning frameworks courses that were not mandated. For the first category, campuses where learning frameworks were mandated, information was frequently made available to students at student engagement events or via advisors who would pass on the information. However, a common answer from respondents was that there was no marketing or advertising because the course was a part of the core agenda. As pertains to marketing of learning frameworks that were not mandated, common techniques included handing out flyers and other materials, attending job/career fairs, and providing information at student orientation. Other strategies included a reliance on advisors, counselors, and faculty to relay information to students and other forms of word-of-mouth advertising.

Training for learning frameworks instructors. Most respondents, 33 (75\%), indicated that training was available for the instructors. The remaining 11 $(25 \%)$ noted that there was either no training available or they were unclear if training was available. The requirement for training, when available, was different depending on the type of instructor. Fifteen responses referenced training specific to "new" instructors and of those, 14 required training with a mix of both ongoing professional development such as pedagogy and student engagement and one-time technical training such as course management software. One response referenced a required biannual training for "fulltime" instructors. Three responses referenced training for "returning" instructors, with only one of those requiring training, such as conference attendance. The largest response set referenced training for "any" instructor $(n=20)$. Ten of the 20 indicated training was required. Types of training mentioned in responses were webinars, brown bag Fridays, online sharing, conferences, mentoring, in-house training, programs such as AVID, and sessions from organizations such as the Dana Center. The frequency of training expectations included never, one-time, annual, biannual, and ongoing.

\section{Curricular Characteristics}

Course topics. Curricular characteristics we examined included the course topics listed in the course calendar of each syllabus obtained from the interviewees. As mentioned in the literature review, the state of Texas requires that these courses address theory, research, and application of the psychology of learning. Therefore, these findings could provide information about the extent to which these courses aligned with this requirement. Of the 44 interviewees, 39 provided a syllabus with sufficient information in their course calendars to include them in the analysis. A total of 930 course topics were extracted, and content analyzed by an expert learning frameworks research/practitioner. The content categories are shown in Appendix C. The category labels were chosen to closely reflect the types of words and phrasing used in the course calendars.

Textbooks. Of the 44 interviewees, 33 (75\%) stated they required a specific textbook for all sections of learning frameworks courses, eight (18\%) reported they did not require a specific textbook, and three stated they were unsure. Of the three interviewees who said they were unsure, one stated they did not always require a common text, the second said text requirements depended on the semester, and the last stated they were currently piloting books to require a common text for the future. Although eight interviewees reported they did not require a common text, two of the eight mentioned they had common texts available for instructors to use if needed. See Table 2 for a list of all texts reported by the interviewees. 
Table 2

Texts Used in Learning Frameworks Courses in Fall 2016 and Spring 2017

\begin{tabular}{|c|c|}
\hline Title (in alphabetical order) & Authors \\
\hline A pocket guide to college success & Shushan \\
\hline $\begin{array}{l}\text { Academic transformations: The road to } \\
\text { college success }\end{array}$ & Sellers, Dochen, and Hodges \\
\hline Becoming a master student & Ellis \\
\hline College and career success & Marsha \\
\hline $\begin{array}{l}\text { College success: A concise practical } \\
\text { guide }\end{array}$ & Strickland and Strickland \\
\hline $\begin{array}{l}\text { College success: Before, during, and } \\
\text { after }\end{array}$ & Raniseski \\
\hline Cornerstones for college success & Sherfield and Moody \\
\hline $\begin{array}{l}\text { Emotional intelligence: Achieving aca- } \\
\text { demic and career excellence in college } \\
\text { and in life }\end{array}$ & Nelson and Low \\
\hline Essential study skills & Wong \\
\hline Motivation in education & Schunk, Meece, and Pintrich \\
\hline On course & Downing \\
\hline $\begin{array}{l}\text { P.O.W.E.R. learning strategies for suc- } \\
\text { cess in college and life }\end{array}$ & Feldmen \\
\hline $\begin{array}{l}\text { Peak performance: Success in college } \\
\text { and beyond }\end{array}$ & Ferret \\
\hline $\begin{array}{l}\text { Student success in college: Doing what } \\
\text { works }\end{array}$ & Harrington \\
\hline The college experience compact & Baldwin, Tietje, and Stoltz \\
\hline Keys to community college success & Carter and Kravits \\
\hline The things they carried & O’Brien \\
\hline $\begin{array}{l}\text { Thriving in college and beyond: Re- } \\
\text { search-based strategies for academic } \\
\text { success and personal development }\end{array}$ & $\begin{array}{l}\text { Cuseo, Thompson, Campag- } \\
\text { na, and Fecas }\end{array}$ \\
\hline UT Dana Center resources & UT Dana Center \\
\hline Your college experience & Gardner and Barefoot \\
\hline Learning framework & $\begin{array}{l}\text { Customized book, Collin } \\
\text { College }\end{array}$ \\
\hline $\begin{array}{l}7 \text { habits of highly effective college } \\
\text { students }\end{array}$ & Covey \\
\hline
\end{tabular}

Assessments. Beyond textbooks, we also asked interviewees the extent to which they used standardized assessments in their learning frameworks courses. Of the 44 respondents, 38 (86\%) reported incorporating standardized assessments in their learning frameworks courses, whereas 6 (14\%) did not. When probed about the types of assessments used, respondents commonly reported using multiple assessments. The three most common types of assessments reported were personality assessments such as the Myers-Briggs Type Indicator, career assessments such as the Strong Interest Inventory, and learning strategies type assessments such as the Learning and Study Strategies Inventory (see Table 3 for a full listing of the types of assessments reported).
Table 3

Types of Assessments Used in Learning Frameworks Courses.

\begin{tabular}{lc}
\hline Source & $f$ \\
\hline Personality & 21 \\
Career & 15 \\
Learning Strategies & 13 \\
College Success & 11 \\
Learning Styles / Preferences & 11 \\
Multiple Intelligences & 4 \\
Emotional Assessments & 3 \\
Other / Miscellaneous & 12 \\
\hline Note. Some interviewees listed multiple assessments.
\end{tabular}

\section{Discussion}

The current study helps to document a surge in learning frameworks course offerings across the state and to recognize both similarities and differences in course characteristics. Our findings support the notion that learning frameworks courses are a valued and important focus area for most all Texas public community colleges.

Texas' decision to offer formula funding for these courses (Hill, 2000), along with the need to support growing numbers of students entering college who are academically underprepared (Center for Community College Student Engagement [CCCSE], 2016), likely helped to spur the increase in these course offerings. The majority of community colleges within this study began offering these courses only within the last decade. Many institutions have made substantial changes to the administrative (e.g. mandates, paring, credit hours) and curricular (e.g. textbooks, assessment tools, learning outcomes) characteristics of the course.

\section{Administrative Characteristics}

While previous research has focused on the content and goals of learning frameworks courses, differentiating them from orientation, transition, and study skills courses (Cole, et. al., 1997), and demonstrating their effectiveness on learning (as noted in our literature review), this study helps to extend these areas of research by documenting how learning frameworks courses are being implemented to serve the needs of Texas public community colleges. As suggested with the findings of this study, learning frameworks courses are not only offered at 45 out of 50 institutions, but over two-thirds of those interviewed indicated that their learning frameworks courses were mandated for all students or those who are FTIC with less than 12-15 credit hours. 
With the diversity that accompanied the growth in the student population for Texas' postsecondary institutions (THECB, 2018), it was imperative that Texas public community colleges identified and implemented supports that catered to specific subpopulations. This study showed $40 \%$ of coordinators indicated that their institution designed courses for special populations. Of particular interest, three-quarters of institutions designating learning frameworks sections for students enrolled in a developmental course also made it a requirement. This study finding speaks to research highlighting the importance of additional supports for students deemed academically underprepared and or underserved (CCCSE, 2016).

Another finding of the current study showed one-third of responders indicated offering paired courses (i.e. EDUC 1300 with a STEM-related course). The general practice of pairing courses has been supported in literature-and especially paring student success courses with content-based courses such as the Dana Centers' Framework for Mathematics and Collegiate Learning Course (University of Texas at Austin Charles A. Dana Center, 2019). Further, the study findings in regard to advertising the course in general, for special subpopulations, and for paired courses, were expected to have distinct differences based on if the course was required or not required. If a course was mandated, we found that marketing was either nonexistent or promoted by advisors or listings on one's degree plan. In cases where a mandate was not in place, this study showed advisors played a vital role. Interviewees also mentioned the use of advertising via institutional events.

\section{Curricula Characteristics}

This finding indicated that curricula-related characteristics varied by institution. Course topics, textbook choice, and assessment tools were the areas of primary distinction among the learning frameworks courses offered. One main conclusion is that while many of the courses did have somewhat similar course content among institutions (e.g. self-regulatory strategies, goal setting, motivation, metacognition, reading comprehension, strategies for taking notes, etc.), there were some courses not well aligned with the THECB authorized formula funding requirements (see Hill, 2000) or having the presence of theoretical models as the curricular core, based on the course calendar topics listed. Of the 930 topics identified, we also found many topics - while useful and important-were beyond the original intent of the THECB mandated curricula (e.g. careers, communication, financial literacy, and relationships, among many others). We also found controversial topics (e.g., learning styles) promoted within some courses, having sparse research underpinnings.
Although an analysis of each textbook and assessment used is beyond the scope of this study, the findings help to document the variation in the use of these resources, which could be examined in more detail in future research. From some of the textbooks listed that the authors of this study were familiar with, many lacked the theoretical connections to the strategies promotes.

Even with the availability of theory-based textbooks, assessments, instructor manuals, textbook web portals and other supplementary materials used to help assist instructors teaching learning frameworks courses, the need for instructor professional development was not overlooked by institutions. Most coordinators interviewed said that training was available or required for instructors teaching learning frameworks courses. However, training, for some institutions, was often limited to a few days within the beginning of the semester or only for new instructors.

\section{Limitations}

All interview data was self-reported and subject to the interviewees' interpretations and available knowledge at the time of the interview. For example, several interviewees did not have complete information on when their learning frameworks courses were initiated and the types of changes made to their courses. In addition, this study is limited in representing the variations in implementation at each institution. Interviewees, for example, described what was expected in general, but did not know the day-to-day implementation fidelity of each course offering. Similarly, data on the course topics extracted from course calendars represents a single syllabus or syllabus template representative of the various course offerings at a single institution and does not necessarily reflect all syllabi or the actual topics covered during class.

\section{Conclusions, Recommendations, and Future Research}

This study captured a snap-shot of the historical, administrative, and curriculum characteristics of Texas's public community colleges' learning frameworks courses as reported by 44 coordinators (or their designees) during the Spring 2016 and Fall 2017 semesters. Distinctions among courses were especially prevalent in regard to curricula topics and the integration of theoretical perspectives, textbooks, resources, and assessment selections. While instructor training was common among institutions, the length and types of training varied. While the authors understand the need for each institution to meet the needs of their specific student populations with learning frameworks courses, the authors do recommend statewide resources be developed focusing on 
student learning outcomes, curriculum topics, theoretical constructs, and assessments to help foster more standardization that meet the THECB course funding requirements.

Several areas of future research, given the findings, include an investigation of Texas's 4-year institutions' learning frameworks course characteristics. Additionally, a statewide examination of courses' effectiveness on students' academic outcomes (e.g. on retention, graduation rates, for targeted special populations, and for paired courses) would be useful. These investigations would be especially important in helping to meet Texas's strategic plan's overarching goal: "By 2030, at least 60 percent of Texans ages 2534 will have a certificate or degree (THECB, 2018, p. 8).

Additionally, we expect learning frameworks courses to continue to evolve with breakthrough research and theories that address student success such as how technology affects learning. As the world becomes more complex, technologically advanced, and diverse in the twenty-first century, Texas students deserve the very best in learning frameworks instruction.

\section{References}

Agee, K., Hodges, R., \& Castillo, A. (2018). Program management. In R. Flippo, T. W. Bean (Eds.), Handbook for college reading and study strategy research ( $3^{\text {rd }}$ ed., pp. 293-314). New York, NY: Routledge.

Bartoszewski, B. L., \& Gurung, R. A. R. (2015). Comparing the relationship of learning techniques and exam score. Scholarship of Teaching and Learning in Psychology, 1(3), 219-228. http://dx.doi.org/10.1037/ stl0000036

Bird, C. (1931). Effective study habits. New York, NY: Century.

Center for Community College Student Engagement. (2016). Expectations meet reality: The underprepared student and community colleges. Austin, TX: The University of Texas at Austin, College of Education, Department of Educational Administration, Program in Higher Education Leadership. Retrieved from http://www.ccsse.org/docs/Underprepared_ Student.pdf

Cole, L., \& Ferguson, J. M. (1935). Students guide to effective study (Rev. ed.). New York, NY: Farrar \& Rinehart.

Cole, R. P., Babcock, C., Goetz, E. T. \& Weinstein, C. E. (1997, October). An in-depth look at academic success courses. Paper presented at the meeting of the College Reading and Learning Association, Sacramento, CA.
Dana Center Mathematics Pathways (2017). DCMP frameworks for mathematics and collegiate learning: Course overview and learning outcomes. Retrieved from https:// dcmathpathways.org/resources/dcmpframeworks-mathematics-and-collegiatelearning-course-overview-and-learningoutcomes

Dembo, M. H., \& Seli, H. P. (2004). Students' resistance to change in learning strategies courses. Journal of Developmental Education. (27)3, 2-11.

Dunlosky, J., Rawson, K. A., Marsh, E. J., Nathan, M. J., \& Willingham, D. T. (2013). Improving students' learning with effective learning techniques: Promising directions from cognitive and educational psychology. Psychological Science in the Public Interest. 14(1), 4-58. doi: $10.1177 / 1529100612453266$

Ellis, D. B. (1985) Becoming a master student ( $5^{\text {th }}$ ed.). Rapid City, SD: College Survival.

Fong, C. J., Zientek, L. R., Ozel, Z. E. Y., \& Phelps, J. M. (2015). Between and within ethnic differences in strategic learning: A study of developmental mathematics students. Social Psychology Education, 18, 55-74. doi:10.1007/s11218014-9275-5

Frederick, R. W. (1938). How to study handbook. New York, NY: Appleton-Century.

Geller, J., Toftness, A. R., Armstrong, P. I., Carpenter, S. K., Manz, C. L., Coffman, C. R., \& Lamm, M. H. (2018). Study strategies and beliefs about learning as a function of academic achievement and achievement goals. Memory, 26(5), 683-690. doi:10.1080/09658 211.2017.1397175

Hensley, L. C., Wolters, C. A., Won, S., \& Brady, A. C. (2018). Academic probation, time management, and time use in a college success course. Journal of College Reading and Learning, 48(2), 105-123. doi:10.1080/1 0790195.2017 .1411214

Hill, M. A. (2000, March 31). Funding for "Learning Framework" courses [Memorandum to Chief Academic Officers, Public Senior Universities]. Austin, TX: Texas Higher Education Coordinating Board.

Hodges, R. (2014). Autobiography of teaching a learning framework course. In J. L. Higbee (Ed.), Continuing contributions to the field: Essays by the Fellows of the Council of Learning Assistance and Developmental Education Associations (pp. 183-204). In Memory of Martha Maxwell. Boone, NC: National Center for Developmental Education.

Hodges, R., \& Acee, T. W. (2017). The many legacies of Dr. Claire Ellen Weinstein, part I tribute: Learning frameworks courses. In M. C. McConnell (Ed.), Promising practices in developmental education (pp. 43-48). San Marcos, TX: The Education Institute, Texas State University. 
Hodges, R., Sellers, D.E., \& Dochen, C.W. (2001). Implementing a learning framework course. In J. L. Higbee \& P. L. Dwinell (Eds.), NADE monograph: 2001 A developmental odyssey, (pp. 3-13). Warrensburg, MO: National Association for Developmental Education.

Hofer, B. K., \& Yu, S. L. (2003). Teaching self-regulated learning through a "learning to learn" course. Teaching of Psychology, (30)1, 3033. doi:10.1207/S15328023TOP3001_05

Hoops, L. D., \& Artrip, A. (2016). College student success course takers' perceptions of college student effectiveness. Learning Assistance Review, (21)2, 55-67.

Hoops, L. D., Yu, S. L., Burridge, A. B., \& Wolters, C. A. (2015). Impact of a student success course on undergraduate academic outcomes. Journal of College Reading and Learning, 45(2), 123-146. doi:10.1080/107 90195.2015.1032041

Howard, C. M., Moret, L., Faulconer, J., Cannon, T., \& Tomlin, A. (2018). Preparing for college success: Exploring undergraduate students' perceptions of the benefits of a college reading and study skills course through action research. Networks: An Online Journal for Teacher Research, 20(1), 1-18. doi:10.4148/2470-6353.1258

Karp, M. M., Raufman, J., Efthimiou, C., \& Ritze, N. (2017). Revising a college 101 course for sustained impact: Early outcomes. Community College Journal of Research and Practice, 41(1), 42-55. doi:10.1080/106689 26.2016.1152929

Kennett, D. J., \& Reed, M. J. (2009). Factors influencing academic success and retention following a 1st-year post-secondary success course. Educational Research and Evaluation (15)2, 153-166. doi: 10:1080/13803610902804382

Keup, J. R., \& Barefoot, B. O. (2005). Learning how to be a successful student: Exploring the impact of first-year seminars on student outcomes. Journal of the First-Year Experience \& Students in Transition, 17(1), 11-47.

Krippendorff, K. (2004). Content Analysis: An Introduction to its Methodology ( $2^{\text {nd }}$ ed.). Thousand Oaks, CA: Sage.

Maxwell, M. (1997). Improving student learning skills: A new edition. Clearwater, $\mathrm{FL}: \mathrm{H} \& \mathrm{H}$.

Pauk, W. (1962, 1974). How to study in college. Boston, MA: Houghton Mifflin.

Pintrich, P. R., Smith, D. A. F., Garcia, T., \& McKeachie, W. J. (1993). Reliability and predictive validity of the Motivated Strategies for Learning Questionnaire (MSLQ). Educational and Psychological Measurement, 53(3), 801-813.
Robinson, F. P. (1936). Effective study. New Edition. New York, NY: Harper \& Row.

Robinson, F. P. (1941). Diagnostic and remedial techniques for effective study. New York, NY: Harper.

Robinson, F. P. (1946). Effective study. New York, NY: Harper \& Row.

Schunk, D. H. (2012). Learning theories: An educational perspective (6th ed.). Boston, MA: Pearson.

Stahl, N. A., \& Henk, W. A. (1986). Tracing the roots of textbook study systems: An extended historical perspective. In J.A. Niles (Ed.), Solving problems in literacy: Learners, teachers and researchers $-35^{\text {th }}$ yearbook of the National Reading Conference (pp. 366-374). Rochester, NY: National Reading Conference.

Texas Higher Education Coordinating Board (THECB). (2018). Texas public higher education almanac: A profile of state of institutional performance and characteristics. Retrieved from www.thecb. state.tx.us/index.cfm?objectid=629F37F0861F-11E8-AE230050560100A9

Tuckman, B. W., \& Kennedy, G. J. (2011). Teaching learning strategies to increase success of first-term college students. The Journal of Experimental Education, 79(4), 478-504. doi: 10.1080/00220973.2010.512318

University of Texas at Austin Charles A. Dana Center. (2019). Frameworks for mathematics and collegiate learning. Retrieved from https:// www.utdanacenter.org/our-work/highereducation/higher-education-curricularresources/frameworks-mathematics-andcollegiate-learning

Urciuoli, J. A., \& Bluestone, C. (2013). Study skills analysis: A pilot study linking a success and psychology course. Community College Journal of Research and Practice, 37(5), 397401. doi:10.1080/10668926.2012.716386

Wolters, C. A., \& Hoops, L. D. (2015). Self-regulated learning interventions for motivationally disengaged college students. In T. Cleary (Ed.), Self-regulated learning interventions with at-risk youth: Enhancing adaptability, performance, and well-being (pp. 67-88). Washington, DC: American Psychological Association.

Weinstein, C. E., Dierking, D.., Husman, J., Roska, L., Powdrill, L. (1998). The impact of a course in strategic learning on the long-term retention of college students. In J. L. Higbee \& P. L. Dwinnel (Eds.). Developmental education: Preparing successful college students (pp. 8596). Columbia, SC: National Resource Center for the First-Year Experience and Students in Transition. 
Weinstein, C. E., \& Mayer, R. E. (1983). The teaching of learning strategies. Innovation Abstracts, 5(32), 1-4.

Whipple, G. M. (1927). How to study effectively (2nd ed.). Bloomington, IN: Public School. 


\section{Appendix A}

\section{Participating and Non-Participating Texas Community Colleges}

\begin{tabular}{|c|c|c|c|c|c|}
\hline Community College & Campus Name & $\begin{array}{l}\text { Campus } \\
\text { Type }\end{array}$ & LF Program & Interviewed & LF Course Number \\
\hline Alamo College & $\begin{array}{c}\text { Northeast Lakeview } \\
\text { College }\end{array}$ & Multiple & Yes & Yes & EDUC 1300 \\
\hline Alamo College & $\begin{array}{l}\text { Northwest Vista } \\
\text { College }\end{array}$ & Multiple & Yes & Yes & EDUC 1300 \\
\hline Alamo College & Palo Alto College & Multiple & Yes & Yes & EDUC 1300 \\
\hline Alamo College & $\begin{array}{c}\text { San Antonio } \\
\text { College }\end{array}$ & Multiple & Yes & Yes & EDUC 1300 \\
\hline Alamo College & St. Philip's College & Multiple & Yes & No & $\begin{array}{l}\text { EDUC } 1300 \\
\text { PYSC } 1300\end{array}$ \\
\hline Alvin Community College & N/A & Single & Yes & Yes & PSYC 1300 \\
\hline Amarillo College & N/A & Single & Yes & Yes & EDUC 1100 \\
\hline Angelina College & $\mathrm{N} / \mathrm{A}$ & Single & Yes & Yes & $\begin{array}{l}\text { EDUC } 1300 \\
\text { PSYC } 1300\end{array}$ \\
\hline Austin Community College & $\begin{array}{l}\text { Cypress Creek } \\
\text { Campus }\end{array}$ & Multiple & Yes & Yes & $\begin{array}{l}\text { EDUC } 1100 \\
\text { EDUC } 1200 \\
\text { EDUC } 1300\end{array}$ \\
\hline Austin Community College & Eastview Campus & Multiple & Yes & Yes & $\begin{array}{l}\text { EDUC } 1100 \\
\text { EDUC } 1200 \\
\text { EDUC } 1300 \\
\end{array}$ \\
\hline Austin Community College & Elgin Campus & Multiple & Yes & Yes & $\begin{array}{l}\text { EDUC } 1100 \\
\text { EDUC } 1200 \\
\text { EDUC } 1300 \\
\end{array}$ \\
\hline Austin Community College & Hay Campus & Multiple & Yes & Yes & $\begin{array}{l}\text { EDUC } 1100 \\
\text { EDUC } 1200 \\
\text { EDUC } 1300\end{array}$ \\
\hline Austin Community College & Highland Campus & Multiple & Yes & Yes & $\begin{array}{l}\text { EDUC } 1100 \\
\text { EDUC } 1200 \\
\text { EDUC } 1300\end{array}$ \\
\hline Austin Community College & Northridge Campus & Multiple & Yes & Yes & $\begin{array}{l}\text { EDUC } 1100 \\
\text { EDUC } 1200 \\
\text { EDUC } 1300\end{array}$ \\
\hline Austin Community College & Rio Grande Campus & Multiple & Yes & Yes & $\begin{array}{l}\text { EDUC } 1100 \\
\text { EDUC } 1200 \\
\text { EDUC } 1300 \\
\end{array}$ \\
\hline Austin Community College & Riverside Campus & Multiple & Yes & Yes & $\begin{array}{l}\text { EDUC } 1100 \\
\text { EDUC } 1200 \\
\text { EDUC } 1300\end{array}$ \\
\hline Austin Community College & $\begin{array}{l}\text { Round Rock } \\
\text { Campus }\end{array}$ & Multiple & Yes & Yes & $\begin{array}{l}\text { EDUC } 1100 \\
\text { EDUC } 1200 \\
\text { EDUC } 1300\end{array}$ \\
\hline Austin Community College & $\begin{array}{l}\text { San Gabriel } \\
\text { Campus }\end{array}$ & Multiple & Yes & Yes & $\begin{array}{l}\text { EDUC } 1100 \\
\text { EDUC } 1200 \\
\text { EDUC } 1300\end{array}$ \\
\hline Austin Community College & $\begin{array}{l}\text { South Austin } \\
\text { Campus }\end{array}$ & Multiple & Yes & Yes & $\begin{array}{l}\text { EDUC } 1100 \\
\text { EDUC } 1200 \\
\text { EDUC } 1300\end{array}$ \\
\hline Blinn College & $\mathrm{N} / \mathrm{A}$ & Single & Yes & Yes & EDUC 1300 \\
\hline Brazosport College & $\mathrm{N} / \mathrm{A}$ & Single & Yes & Yes & PSYC 1300 \\
\hline Central Texas College & $\mathrm{N} / \mathrm{A}$ & Single & Yes & Yes & PSYC 1300 \\
\hline Cisco College & N/A & Single & Yes & Yes & EDUC 1100 \\
\hline Clarendon College & $\mathrm{N} / \mathrm{A}$ & Single & Yes & Yes & EDUC 1100 \\
\hline Coastal Bend College & Alice Site & Multiple & No & No & N/A \\
\hline Coastal Bend College & Beeville Campus & Multiple & No & No & N/A \\
\hline
\end{tabular}


Appendix A (Continued)

\begin{tabular}{|c|c|c|c|c|c|}
\hline Coastal Bend College & Kingsville Site & Multiple & No & No & $\mathrm{N} / \mathrm{A}$ \\
\hline Coastal Bend College & Pleasanton Site & Multiple & No & No & $\mathrm{N} / \mathrm{A}$ \\
\hline College of the Mainland & N/A & Single & Yes & Yes & $\begin{array}{l}\text { EDUC } 1300 \\
\text { PSYC } 1300\end{array}$ \\
\hline Collin College & Allen Center & Multiple & Yes & Yes & $\begin{array}{l}\text { EDUC } 1300 \\
\text { PSYC } 1100 \\
\text { PSYC } 1300\end{array}$ \\
\hline Collin College & $\begin{array}{c}\text { Central Park } \\
\text { Campus }\end{array}$ & Multiple & Yes & Yes & $\begin{array}{l}\text { EDUC } 1300 \\
\text { PSYC } 1100 \\
\text { PSYC } 1300\end{array}$ \\
\hline Collin College & Courtyard Center & Multiple & Yes & Yes & $\begin{array}{l}\text { EDUC } 1300 \\
\text { PSYC } 1100 \\
\text { PSYC } 1300\end{array}$ \\
\hline Collin College & $\begin{array}{l}\text { Preston Ridge } \\
\text { Campus }\end{array}$ & Multiple & Yes & Yes & $\begin{array}{l}\text { EDUC } 1300 \\
\text { PSYC } 1100 \\
\text { PSYC } 1300\end{array}$ \\
\hline Collin College & Rockwall Center & Multiple & Yes & Yes & $\begin{array}{l}\text { EDUC } 1300 \\
\text { PSYC } 1100 \\
\text { PSYC } 1300\end{array}$ \\
\hline Collin College & $\begin{array}{l}\text { Spring Creek } \\
\text { Campus }\end{array}$ & Multiple & Yes & Yes & $\begin{array}{l}\text { EDUC } 1300 \\
\text { PSYC } 1100 \\
\text { PSYC } 1300\end{array}$ \\
\hline $\begin{array}{c}\text { Dallas County Community } \\
\text { College District }\end{array}$ & $\begin{array}{l}\text { Brookhaven } \\
\text { College }\end{array}$ & Multiple & Yes & Yes & $\begin{array}{l}\text { EDUC } 1300 \\
\text { PSYC } 1300\end{array}$ \\
\hline $\begin{array}{c}\text { Dallas County Community } \\
\text { College District }\end{array}$ & $\begin{array}{l}\text { Cedar Valley } \\
\text { College }\end{array}$ & Multiple & Yes & Yes & $\begin{array}{l}\text { EDUC } 1300 \\
\text { PYSC } 1300\end{array}$ \\
\hline $\begin{array}{c}\text { Dallas County Community } \\
\text { College District }\end{array}$ & Eastfield College & Multiple & Yes & Yes & $\begin{array}{l}\text { EDUC } 1300 \\
\text { PYSC } 1300\end{array}$ \\
\hline $\begin{array}{c}\text { Dallas County Community } \\
\text { College District }\end{array}$ & El Centro College & Multiple & Yes & Yes & $\begin{array}{l}\text { EDUC } 1300 \\
\text { PYSC } 1300\end{array}$ \\
\hline $\begin{array}{c}\text { Dallas County Community } \\
\text { College District }\end{array}$ & $\begin{array}{l}\text { Mountain View } \\
\text { College }\end{array}$ & Multiple & Yes & Yes & $\begin{array}{l}\text { EDUC } 1300 \\
\text { PYSC } 1300\end{array}$ \\
\hline $\begin{array}{l}\text { Dallas County Community } \\
\text { College District }\end{array}$ & North Lake College & Multiple & Yes & Yes & $\begin{array}{l}\text { EDUC } 1300 \\
\text { PYSC } 1300\end{array}$ \\
\hline $\begin{array}{c}\text { Dallas County Community } \\
\text { College District }\end{array}$ & Richland College & Multiple & Yes & Yes & $\begin{array}{l}\text { EDUC } 1300 \\
\text { PYSC } 1300\end{array}$ \\
\hline Del Mar College & $N / A$ & Single & Yes & No & $\begin{array}{l}\text { EDUC } 1300 \\
\text { PYSC } 1300\end{array}$ \\
\hline El Paso Community College & N/A & Single & Yes & Yes & EDUC 1300 \\
\hline Frank Phillips College & $\mathrm{N} / \mathrm{A}$ & Single & Yes & Yes & $\begin{array}{l}\text { EDUC } 1100 \\
\text { PSYC } 1100\end{array}$ \\
\hline Galveston College & N/A & Single & Yes & No & $\begin{array}{l}\text { EDUC } 1300 \\
\text { PYSC } 1300\end{array}$ \\
\hline Grayson College & N/A & Single & Yes & Yes & $\begin{array}{l}\text { EDUC } 1300 \\
\text { PSYC } 1300\end{array}$ \\
\hline Hill College & $\mathrm{N} / \mathrm{A}$ & Single & Yes & Yes & $\begin{array}{l}\text { PSYC } 1100 \\
\text { PSYC } 1300\end{array}$ \\
\hline $\begin{array}{l}\text { Houston Community College } \\
\text { System }\end{array}$ & Central & Multiple & Yes & Yes & EDUC 1300 \\
\hline $\begin{array}{l}\text { Houston Community College } \\
\text { System }\end{array}$ & Northeast & Multiple & Yes & No** & EDUC 1300 \\
\hline $\begin{array}{l}\text { Houston Community College } \\
\text { System }\end{array}$ & Northwest & Multiple & Yes & No & EDUC 1300 \\
\hline $\begin{array}{l}\text { Houston Community College } \\
\text { System }\end{array}$ & Southeast & Multiple & Yes & No & EDUC 1300 \\
\hline $\begin{array}{l}\text { Houston Community College } \\
\text { System }\end{array}$ & Southwest & Multiple & Yes & No & EDUC 1300 \\
\hline Howard College & N/A & Single & Yes & Yes & $\begin{array}{l}\text { EDUC } 1100 \\
\text { PSYC } 1100\end{array}$ \\
\hline
\end{tabular}




\section{Appendix A (Continued)}

\begin{tabular}{|c|c|c|c|c|c|}
\hline Kilgore College & $\mathrm{N} / \mathrm{A}$ & Single & Yes & Yes & $\begin{array}{l}\text { EDUC } 1100 \\
\text { EDUC } 1300\end{array}$ \\
\hline Laredo Community College & $\mathrm{N} / \mathrm{A}$ & Single & Yes & No & \\
\hline Lee College & $\mathrm{N} / \mathrm{A}$ & Single & Yes & Yes & EDUC 1200 \\
\hline Lone Star College & CyFair & Multiple & Yes & No & EDUC 1300 \\
\hline Lone Star College & Kingwood Campus & Multiple & Yes & No & EDUC 1300 \\
\hline Lone Star College & $\begin{array}{l}\text { Montgomery } \\
\text { Campus }\end{array}$ & Multiple & Yes & No & EDUC 1300 \\
\hline Lone Star College & $\begin{array}{l}\text { North Harris } \\
\text { Campus }\end{array}$ & Multiple & Yes & No & EDUC 1300 \\
\hline Lone Star College & Tomball Campus & Multiple & Yes & No & EDUC 1300 \\
\hline Lone Star College & $\begin{array}{l}\text { University Park } \\
\text { Campus }\end{array}$ & Multiple & Yes & No & EDUC 1300 \\
\hline McLennan Community College & $\mathrm{N} / \mathrm{A}$ & Single & Yes & Yes & $\begin{array}{l}\text { EDUC } 1100 \\
\text { PSYC } 1100 \\
\text { EDUC } 1300 \\
\text { PSYC } 1300\end{array}$ \\
\hline Midland College & $\mathrm{N} / \mathrm{A}$ & Single & Yes & No & EDUC 1100 \\
\hline Navarro College & $\mathrm{N} / \mathrm{A}$ & Single & No & No & $\mathrm{N} / \mathrm{A}$ \\
\hline North Central Texas College & Bowie Campus & Multiple & Yes & No & $\begin{array}{l}\text { EDUC } 1300 \\
\text { PSYC } 1300\end{array}$ \\
\hline North Central Texas College & Corinth Campus & Multiple & Yes & No & $\begin{array}{l}\text { EDUC } 1300 \\
\text { PSYC } 1300\end{array}$ \\
\hline North Central Texas College & $\begin{array}{l}\text { Flower Mound } \\
\text { Campus }\end{array}$ & Multiple & Yes & No & $\begin{array}{l}\text { EDUC } 1300 \\
\text { PSYC } 1300\end{array}$ \\
\hline North Central Texas College & Gainsville Campus & Multiple & Yes & No & $\begin{array}{l}\text { EDUC } 1300 \\
\text { PSYC } 1300\end{array}$ \\
\hline North Central Texas College & Graham Campus & Multiple & Yes & No & $\begin{array}{l}\text { EDUC } 1300 \\
\text { PSYC } 1300 \\
\end{array}$ \\
\hline $\begin{array}{c}\text { Northeast Texas Community } \\
\text { College }\end{array}$ & $\mathrm{N} / \mathrm{A}$ & Single & Yes & Yes & EDUC 1300 \\
\hline Odessa College & $\mathrm{N} / \mathrm{A}$ & Single & Yes & Yes & COLL 0171 \\
\hline Panola College & $\mathrm{N} / \mathrm{A}$ & Single & No & No & $\mathrm{N} / \mathrm{A}$ \\
\hline Paris Junior College & $\mathrm{N} / \mathrm{A}$ & Single & Yes & Yes & $\begin{array}{l}\text { EDUC } 1300 \\
\text { PSYC } 1300 \\
\end{array}$ \\
\hline Ranger College & $\mathrm{N} / \mathrm{A}$ & Single & Yes & Yes & $\begin{array}{l}\text { EDUC } 1100 \\
\text { PSYC } 1100\end{array}$ \\
\hline San Jacinto College & Central Campus & Multiple & Yes & Yes & $\begin{array}{l}\text { EDUC } 1300 \\
\text { PSYC } 1300\end{array}$ \\
\hline San Jacinto College & North Campus & Multiple & Yes & Yes & $\begin{array}{l}\text { EDUC } 1300 \\
\text { PSYC } 1300\end{array}$ \\
\hline San Jacinto College & South Campus & Multiple & Yes & Yes & $\begin{array}{l}\text { EDUC } 1300 \\
\text { PSYC } 1300\end{array}$ \\
\hline South Plains College & N/A & Single & Yes & Yes & $\begin{array}{c}\text { EDUC } 1100 \text { EDUC } \\
1300 \\
\end{array}$ \\
\hline South Texas College & $\mathrm{N} / \mathrm{A}$ & Single & Yes & Yes & $\begin{array}{c}\text { EDUC } 1300 \text { PSYC } \\
1300 \\
\end{array}$ \\
\hline Southwest Texas Junior College & N/A & Single & Yes & Yes & $\begin{array}{c}\text { EDUC } 1100 \text { EDUC } \\
1300 \\
\end{array}$ \\
\hline Tarrant County College & Connect Campus & Multiple & No & Yes* & $\mathrm{N} / \mathrm{A}$ \\
\hline Tarrant County College & Northeast Campus & Multiple & No & No & $\mathrm{N} / \mathrm{A}$ \\
\hline Tarrant County College & Northwest Campus & Multiple & No & No & $\mathrm{N} / \mathrm{A}$ \\
\hline Tarrant County College & South Campus & Multiple & No & No & $\mathrm{N} / \mathrm{A}$ \\
\hline Tarrant County College & Southeast Campus & Multiple & No & No & $\mathrm{N} / \mathrm{A}$ \\
\hline Tarrant County College & $\begin{array}{c}\text { Trinity River } \\
\text { Campus }\end{array}$ & Multiple & No & No & $\mathrm{N} / \mathrm{A}$ \\
\hline
\end{tabular}


Appendix A (Continued)

\begin{tabular}{|c|c|c|c|c|c|}
\hline Temple College & N/A & Single & Yes & Yes & $\begin{array}{l}\text { EDUC } 1300 \\
\text { PSYC } 1300\end{array}$ \\
\hline Texarkana College & N/A & Single & Yes & Yes & PSYC 1300 \\
\hline Texas Southmost College & N/A & Single & No & No & $\mathrm{N} / \mathrm{A}$ \\
\hline $\begin{array}{l}\text { Trinity Valley Community } \\
\text { College }\end{array}$ & $\mathrm{N} / \mathrm{A}$ & Single & Yes & Yes & $\begin{array}{l}\text { EDUC } 1300 \\
\text { PSYC } 1300\end{array}$ \\
\hline Tyler Junior College & N/A & Single & Yes & Yes & $\begin{array}{l}\text { EDUC } 1300 \\
\text { PSYC } 1300\end{array}$ \\
\hline Vernon College & N/A & Single & Yes & Yes & EDUC 1300 \\
\hline Victoria College & N/A & Single & Yes & Yes & EDUC 1300 \\
\hline Weatherford College & $\mathrm{N} / \mathrm{A}$ & Single & Yes & Yes & EDUC 1300 \\
\hline Western Texas College & N/A & Single & Yes & Yes & $\begin{array}{l}\text { EDUC } 1100 \\
\text { EDUC } 1300 \\
\text { PSYC } 1300\end{array}$ \\
\hline Wharton County Junior College & N/A & Single & Yes & Yes & PSYC 1300 \\
\hline
\end{tabular}

Note. ${ }^{*}$ This campus director was interviewed but the course was not recognized as learning frameworks with a course prefix and number of STSC 0111.

** The TACC (Texas Association of Community Colleges) represented Houston Community College as one large system; therefore, one director was interviewed.

Appendix B

Interview Questions Used in the Study

Historical Questions

- When did you first begin offering learning frameworks courses?

- Has your learning frameworks program/courses changed over the years?

o If so, in what ways (e.g., credit hours, curriculum)?

Administrative Questions

- Are there sections designed for special populations or disciplines?

- If so, what are those special populations or disciplines?

- Are any of your learning framework courses designed specifically for students enrolled in developmental education courses?

- Are these courses mandatory for all students?

o Who are the courses mandated for?

- $\quad$ Are your Learning Frameworks course sections paired with another course?

o If yes, with what course(s) is the Learning Frameworks course paired?

- How are these courses marketed/advertised?

- Is training available or required of instructors?

o If so, please describe.

Curricular questions

- Does your learning framework course require one specific textbook or textbooks for all sections?

o If yes, what are the titles and authors of the textbook(s) you use.

o If no, can you tell me which textbooks your instructors commonly use? (most common, second most common, etc.).

- Does your learning framework course incorporate standardized assessments (e.g. learning strategies assessments or personality inventories)?

o What assessments do you use? 


\section{Appendix C}

Categories of Course Topics Listed in Learning Frameworks Course Calendars in Fall 2016 and Spring 2017

1. Academic integrity / ethics

2. Academic planning / advising

3. Campus introduction / resources / policies

4. Career

a. Career (in general)

b. Career and major

c. Career exploration

d. Career seeking

5. Communication

a. Academic communication / discourse

b. Communication (in general)

c. Oral communication

6. Diversity / inclusivity

7. Emotional intelligence

8. Financial

a. Financial aid / scholarships

b. Financial literacy / money / money management

9. Habits

10. Information literacy / library resources

11. Intelligence / multiple intelligence

12. Leadership

13. Learning and cognition
a. Brain-based learning
b. Learning / cognition (in general)
c. Lifelong learning
d. Learning strategies
e. Learning styles / preferences
f. Learning theories / models
g. Memory
h. Thinking strategies
i. Creative thinking
ii. Critical thinking
iii. Decision making
iv. Problem solving

14. Literacy

a. Reading strategies / skills

b. Writing strategies / skills

15. Metacognition

16. Motivation
a. Attitudes / values
b. Expectations / beliefs
c. Goals and goal-setting
d. Motivation (in general)

17. Note taking

18. Relationships / support structures / interdependence

19. Responsibility

20. Self-awareness / self-reflection

21. Self-change / transformation

22. Self-management / self-regulation

23. Stress / stress management

24. Studying / study skills

25. Success

26. Technological skills / attitudes towards technology

27. Test anxiety

28. Test strategies

29. Time management

30. Wellness 


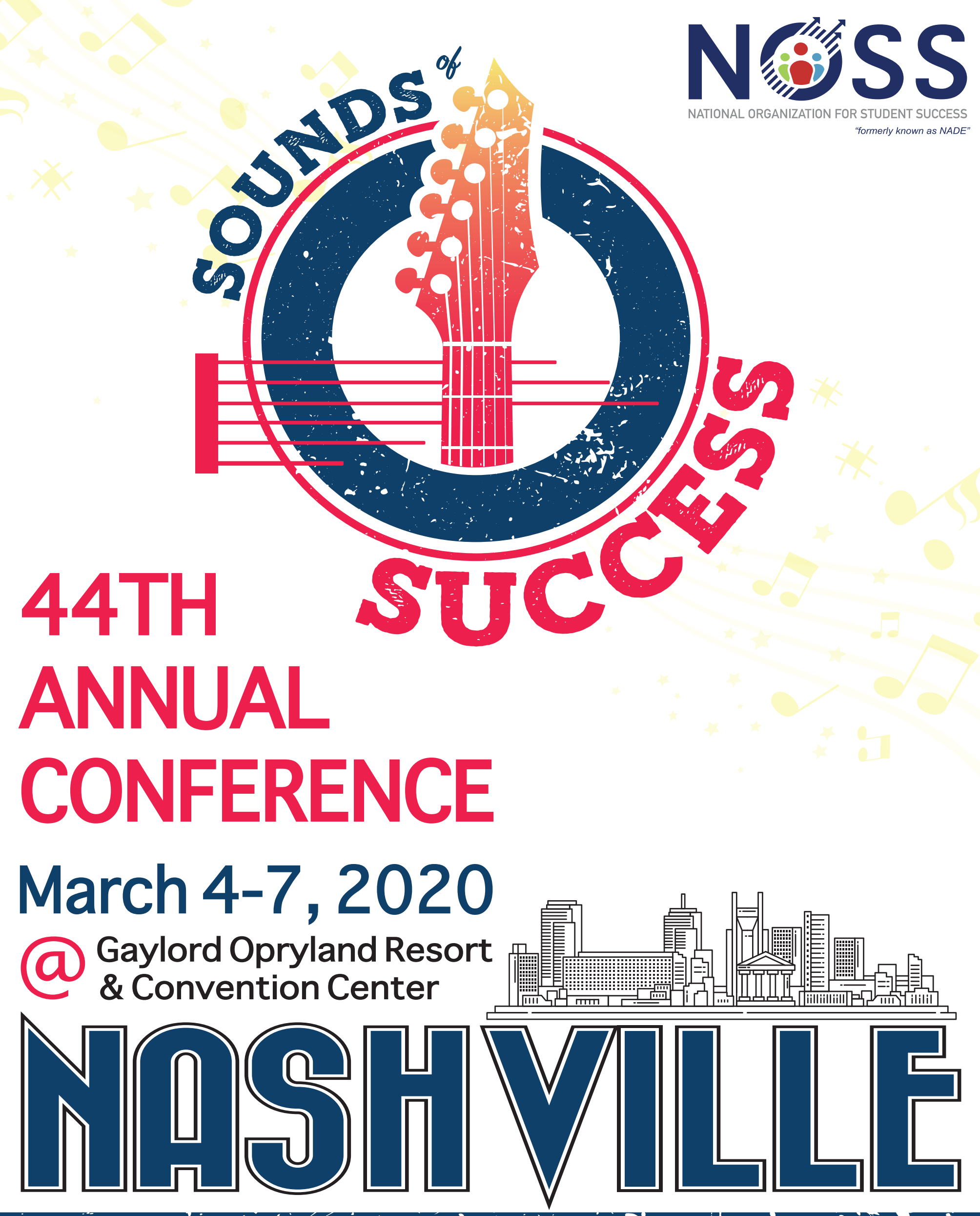

\# WWW THENADE

NAME, CO-CHAIR@EMAIL.COM
NAME, CO-CHAIR@EMAIL.COM

$\because \quad \because$ 


\title{
First-Year Seminars: A Comparison
}

\section{of Course Characteristics and High} Impact Practices at Two-Year Colleges

\author{
Forrest C. Lane, Sam Houston State University \\ Andrew P. Miller, Sam Houston State University
}

\section{ABSTRACT}

Contemporary literature underscores the salience of First-Year seminars (FYS) in supporting the success of college students. The problem is that the research outlining the characteristics of FYS at two-year colleges is sparse, particularly when certain populations are required to enroll in these courses. There is little information about how these seminars vary to meet the needs of certain students. This study examined the characteristics of FYS at two-year institutions where academically underprepared students were required to enroll in a first-year seminar. The results and implications for practice are discussed in this study.

.

here is a need to increase the number of students nationally who earn degrees and certificates. The state of Texas reported it will need "approximately 60 percent of 25- to 34-year-olds to hold a quality certificate or degree by 2030" in order to remain globally competitive (Texas Higher Education Coordinating Board, 2015, p. 2). Persistence and degree completion rates of college students remain low among two-year colleges where only "30 percent of first-time full-time students earn a certificate or associate degree within three years" (Karp, Raufman, Efthimiou, \& Ritze, 2015, p. 1). These rates are even lower among students who are placed into developmental education. More than half of first-time enrolled students who enter a two-year college and test into a developmental course do not make it through developmental course sequences (Bailey, Jeong, \& Cho, 2010). This makes interventions that target academically underprepared students important toward meeting long-term strategic goals for colleges.

Institutions have responded by offering firstyear seminars as a tool to provide students with the academic and social support needed to be successful in college. These seminars support student transition into college by developing academic skills (e.g., study skills, writing skills, and time management), introducing students to campus resources, and facilitating collaborative partnerships (faculty, staff, and students). The problem is that there are different types of first-year seminars (extended orientations, academic seminars, etc.), and not all seminars types are correlated with the same student outcomes (Per- mzadian \& Credé, 2016). This may differentially affect the success of academically underprepared students. Unfortunately, limited information exists about how characteristics of first-year seminars vary in two-year colleges when academically underprepared students are required to enroll in them. Assessing the alignment of seminar type and institutional outcomes may be important to student success. This study explored differences in the characteristics and high-impact practices of first-year seminars at two-year colleges that required academically underprepared students to enroll in them. Results may help to inform how first-year seminars currently meet the needs of underprepared students at these institutions.

\section{Literature Review}

Earning a high school diploma does not guarantee that a student will be ready for college (Strong American Schools, 2008). As many as 30\% of all firstor second-year undergraduate students at four-year colleges are required to take developmental or remedial courses prior to enrollment in college-level English and mathematics (Radwin, Wine, Siegel, \& Bryan, 2013). Due to the open-access mission of twoyear colleges, students are placed into developmental courses at even higher rates within these institutions. This often results in significant costs to both students and the institutions that serve them. Strong American Schools (2008) estimated that remedial courses cost community colleges between $\$ 1.9$ and $\$ 2.3$ billion dollars annually. Further, students enrolled in developmental education were reported to 
have lower degree attainment rates in comparison to those who do not enroll in developmental education (What Works Clearinghouse, 2016; Levin \& Calcagno, 2008). Students enrolled in developmental courses often need additional academic support to be successful in college.

Learning communities are one approach that can be used to support the academic success of underprepared students. Learning communities are small groups of students or cohorts who are placed together based on shared common academic goals. Higher education professionals implement these learning communities to improve relationships with other students and faculty, facilitate greater levels of academic engagement, and improve the level of support provided to students. Learning communities are reported to have a positive effect on the persistence of college students (Bailey \& Alfonso, 2005).

First-year seminars represent one type of learning community and are broadly defined as "a course intended to enhance the academic and/ or social integration of first-year students" (Barefoot, 1992, p. 49). The origins of these seminars date back more than a century but gained momentum in the 1980s as accountability became increasingly important for institutions. irst-year seminars were seen as a way to help students successfully transition into the college and university environment. Various names have been used to describe these seminars over the years, including freshman orientation courses, student success courses, and first-year seminars. The use of the term seminar is more contemporary and "reflects an increasing rigor and acceptance in the academy" of these courses (Hunter \& Linder, 2005, p. 279). Some institutions have expanded even further the academic focus of these seminars by developing frameworks courses, such as the Frameworks for Mathematics and Collegiate Learning course at the University of Texas. Frameworks is also a first-year seminar but incorporates theory from psychology and the learning sciences and is credit bearing (Charles A. Dana Center, 2014a). However, these variations illustrate that seminars can differ in terms of the curriculum, awarding of college credit, and number of credit hours, in addition to the titles used to describe them. The term first-year seminar is thus used here and throughout the paper to refer more broadly the collection of these courses, which is consistent with the higher education literature and national surveys used to examine them.

First-year seminars are best distinguished from one another based on course characteristics or the seminar type offered by the institution. Barefoot (1992) offered a typology of first-year seminars based on course characteristics that is still commonly used today. The types of first-year seminars in that typology included a) extended orientation seminars, b) academic seminars with generally uniform academic content, c) academic seminars on various topics, d) paraprofessional seminars, and e) basic study skills seminars. The most common type of first-year seminar were those that served as an extended orientation, although the use of academic seminars (e.g., Frameworks for Mathematics and Collegiate Learning) has increased more than any other seminar type in recent years (Young, \& Hopp, 2014). Extended orientation seminars typically provide access to information about "campus resources, college policies, and procedures, basic study skills, time management, and learning strategies" (Permzadian \& Credé, 2016, p. 286). Academic seminars commonly include content that promotes the development of skills such as writing, reading, and critical thinking. Successful seminars offer academic credit, collaboratively include faculty and staff in the development of these seminars, provide instructor training and compensation, involve upper-level students in course delivery, and actively assess course effectiveness (Hunter \& Linder, 2005).

Research indicates a positive relationship exists between first-year seminars and both persistence (Lang, 2007; Pascarella \& Terenzini, 2005; Porter \& Swing, 2006; Schnell \& Doetkott, 2003;) and grade performance (Blackett, 2008; DeRoma, Bell, Zaremba \& Albee, 2005), but those relationships are also moderated by the type of seminar offered by the institution (Permzadian \& Credé, 2016). Not all seminar types are correlated with the same student outcomes. It was recommended that institutions seeking to improve student retention design first-year seminars with an orientation focus. In contrast, institutions that seek to improve the academic performance of college students should design first-year seminars with an academic component.

According to national data, $86 \%$ of two-year institutions and $91 \%$ of four-year institutions offered some type of first-year seminar in 2012-2013 (Young \& Hopp, 2014). Despite the increased availability of first-year seminars at colleges, "31\% of two-year campuses required fewer than $10 \%$ of their students to take a first-year seminar" (Young \& Hopp, 2014, p. 12). This may be the result of pressure to avoid 
mandating additional courses for students who are already required to take other non-credit bearing courses (i.e., developmental or remedial courses). This may also be a function of the differentiated ways in which first-year seminars are integrated into the academic curriculum. Some institutions include firstyear seminars as part of the core curriculum (e.g., Frameworks) while other institutions offer them only as an elective course. When first-year seminars are elective or outside the student's degree plan, the students most likely to benefit from these courses may be the least likely to enroll in them.

Students who come to college academically underprepared often need additional academic support and may benefit from a first-year seminar with an academic component, particularly given that the retention of these students is dependent upon on their academic performance. However, little information exists about the characteristics of these seminars at two-year colleges when academically underprepared students are required to take these courses. Given the number of students who come to college academically underprepared, the lack of national survey data disaggregated in this way is concerning. The purpose of this study was to explore how the characteristics and high-impact practices varied among two-year colleges that required academically underprepared students to enroll in the institution's first-year seminar and those that did not. This may help to inform the alignment between seminar type and the needs of academically underprepared students at two-year colleges.

\section{Sample}

\section{Method}

Data for this study were obtained from the 2012-13 National Survey of First-Year Seminars (NSFYS) (Young and Hopp, 2014). This survey collected data on first-year seminar programming from Chief Academic Officers, Chief Executive Officers, or Chief Student Affairs Officers at accredited colleges and universities across the United States. Of those invited to participate, 896 responded to the survey ( $24 \%$ response rate). The data were then limited to two-year colleges ( $n=206$ ) given that these institutions serve the greatest proportion of students who are academically underprepared or who are required to enroll in developmental courses.

The demographic characteristics of the twoyear colleges included in this study are reported in Table 1. Most of these institutions were public (93.2\%) with first-year class sizes of 1,000 or more students. The percent of two-year institutions that offered any type of first-year seminar was $86.4(n=178)$ although not all of these colleges provided information about populations of students required to enroll in these seminars. Of the 128 institutions that provided this information, $30 \%$ reported that they required academically underprepared students to enroll in a first-year seminar $(n=38)$. Only $20 \%$ of institutions required students placed into a developmental or remedial course to enroll in a first-year $(n=26)$.

Table 1

Characteristics of First-Year Seminars at Two-Year Colleges $(N=206)$

\begin{tabular}{|c|c|c|}
\hline & $n$ & $\%$ \\
\hline \multicolumn{3}{|l|}{ Institutional Control } \\
\hline Public & 192 & 93.2 \\
\hline Private & 14 & 6.8 \\
\hline \multicolumn{3}{|l|}{ First-Year Class Size } \\
\hline 500 or less & 33 & 16.0 \\
\hline $501-1,000$ & 40 & 19.4 \\
\hline $1,001-2,000$ & 49 & 23.8 \\
\hline $2,001-4,000$ & 49 & 23.8 \\
\hline More than 4,000 & 35 & 17.0 \\
\hline \multicolumn{3}{|l|}{ First-Year Seminar } \\
\hline Yes & 178 & 86.4 \\
\hline No & 28 & 13.6 \\
\hline \multicolumn{3}{|c|}{ Approximate Percentage of Students Enrolled in FYS } \\
\hline Less than $10 \%$ & 28 & 13.6 \\
\hline $10-19 \%$ & 21 & 10.2 \\
\hline $20-29 \%$ & 17 & 8.3 \\
\hline $30-39 \%$ & 16 & 7.8 \\
\hline $40-49 \%$ & 7 & 3.4 \\
\hline $50-59 \%$ & 18 & 8.7 \\
\hline $60-69 \%$ & 11 & 5.3 \\
\hline $70-79 \%$ & 14 & 6.8 \\
\hline $80-89 \%$ & 15 & 7.3 \\
\hline $90-99 \%$ & 22 & 10.7 \\
\hline $100 \%$ & 6 & 2.9 \\
\hline \multicolumn{3}{|c|}{ Students Required to Participate in FYS } \\
\hline Developmental Education & 26 & 29.7 \\
\hline Academically Underprepared & 38 & 20.3 \\
\hline
\end{tabular}

\section{Variables}

Because requirements for developmental education vary by state (some states allow students to opt out of remedial education courses), and students placed into developmental courses can be considered academically underprepared, groups were compared on this marker in the dataset to increase the comparative sample size in this study. This resulted in a sample size of 128 two-year 
colleges, of which 38 required academically underprepared students to enroll in a first-year seminar and 90 did not require these students to enroll in a first-year seminar.

Institutions were asked about the (a) types of seminars, (b) objectives, and (c) topics included as a part of their first-year seminars. Institutions were able to select all discrete types of seminars (extended orientations, academic seminars with generally uniform content across sections, academic seminars on various topics, preprofessional seminars, and basic study skills seminars) offered at that institution's campus. The NSFYS also asked institutions to identify the three most important course objectives and three most important course topics covered by the seminar. Objectives and topics were selected from a pre-populated list available in Appendix B of Young \& Hopp (2014). Course objectives included items such as developing academic skills, increasing student-faculty interaction, and developing a connection with the institution. Course topics included items such time management, writing skills, and critical thinking skills.

Institutions were also asked about the use of high-impact practices (HIPs) within their first-year seminar. HIPs are those practices shown to lead to "deep learning, first-year GPA, and first- to second-year retention as well as gains in 21st century learning outcomes identified through AAC\&U's [Association of American College and Universities] LEAP initiative" (Young \& Hopp, 2014). The 2012-2013 NSFYS included seven HIPs most-applicable to first-year students. These HIPs included writing-intensive experiences, collaborative assignments and projects, diversity and global learning, service-learning, learning communities, common reading experiences, and undergraduate research opportunities.

\section{Analysis}

Institutions requiring academically underprepared students to enroll in a first-year seminar and institutions that did not were compared using chi-square test of independence $(\alpha=.05)$. The chi-square test statistic can be less accurate when small samples are used in the analysis. As such, the probability value for each chisquare test was computed using Fisher's exact test since this method is a better approximation of the chi-square distribution within small samples (Field, 2013). Odds ratios were also used as a measure of effect size for each chi-square test. Prior to interpreting the odds ratios associated with these comparisons, all ratios below 1.0 were inverted to improve interpretation (Osborne, 2006). These inversions are interpreted as times less likely.

\section{Results}

Two-year colleges that required academically underprepared students to enroll in a first-year seminar were 5.60 times more likely to offer a basic study skills seminar than two-year colleges that did not require these students to enroll in a first-year seminar $\left(\chi^{\wedge} 2[1]=18.54\right.$, $p<.001)$. Basic study skills courses tend to focus on note taking, tests-taking strategies, and critical reading techniques (Young \& Hopp, 2014). These same colleges were also 3.7 times more likely to offer a hybrid seminar ( $\chi^{\wedge} 2[1]$ $=7.28, p=.014)$ and 3.8 times more likely to offer a discipline linked seminar $\left(\chi^{\wedge} 2[1]=5.21, p=.030\right)$. Discipline linked seminars are usually intended to prepare students for the expectations of a certain career or profession. In contrast, two-year colleges that required academically underprepared students to enroll in a first-year seminar were 4.2 times less likely to offer a seminar that served as an extended orientation $\left(\chi^{\wedge} 2[1]=11.99, p=.001\right)$.

Table 2

Primary First-Year Seminar Type $(n=128)$

\begin{tabular}{|c|c|c|c|c|c|c|c|}
\hline \multirow[b]{3}{*}{ Seminar Type } & \multicolumn{7}{|c|}{ Academically Underprepared Students in FYS } \\
\hline & \multicolumn{2}{|c|}{ Not Required } & \multicolumn{2}{|c|}{ Required } & \multirow[b]{2}{*}{$\%$ Diff } & \multirow[b]{2}{*}{$\chi^{2}$} & \multirow[b]{2}{*}{ OR } \\
\hline & $n$ & $\%$ & $n$ & $\%$ & & & \\
\hline \multicolumn{8}{|c|}{ Percentages larger for institutions requiring students to attend } \\
\hline Basic study skills & 23 & 25.6 & 25 & 65.8 & 40.2 & $18.5^{* *}$ & 5.6 \\
\hline Hybrid & 9 & 10.0 & 11 & 28.9 & 18.9 & $7.3^{*}$ & 3.7 \\
\hline Pre-professional or discipline linked & 5 & 5.6 & 7 & 18.4 & 12.8 & 5.2 & 3.8 \\
\hline Academic: uniform content & 21 & 23.3 & 12 & 31.6 & 8.3 & 0.9 & 1.5 \\
\hline \multicolumn{8}{|c|}{ Percentages lower for institutions requiring students to attend } \\
\hline Academic: various topics & 9 & 10.0 & 1 & 2.6 & -7.4 & 2.0 & $0.2(4.2)$ \\
\hline Extended orientation & 74 & 82.2 & 20 & 52.6 & -30.4 & $12.0^{* *}$ & $0.2(4.2)$ \\
\hline
\end{tabular}


Two-year colleges were then compared on the course objectives and course topics of their first-year seminars. Some comparisons could not be made because of the limited number of colleges that identified a particular course objective as being most important. For example, none of the colleges in this sample identified oral communications skills as a primary objective. Among the objectives and topics that could be compared, two-year colleges that required academically underprepared students to enroll in a first-year seminar were 3.6 times more likely to focus on developing academic skills $\left(\chi^{\wedge} 2[1]=3.59, p=.047\right)$. These institutions were also 4.8 times less likely to focus on creating a common first-year experience $\left(\chi^{\wedge} 2[1]=4.75, p=.022\right)$. There were no statistical differences found between groups on any of the other course objectives or course topics.

Table 3

First-Year Seminar Course Objectives $(n=120)$

\begin{tabular}{|c|c|c|c|c|c|c|c|}
\hline \multirow[b]{3}{*}{ Course Objective } & \multicolumn{7}{|c|}{ Academically Underprepared Students in FYS } \\
\hline & \multicolumn{2}{|c|}{ Not Required } & \multicolumn{2}{|c|}{ Required } & \multirow[b]{2}{*}{$\%$ Diff } & \multirow[b]{2}{*}{$\chi^{2}$} & \multirow[b]{2}{*}{ OR } \\
\hline & $n$ & $\%$ & $n$ & $\%$ & & & \\
\hline \multicolumn{8}{|l|}{ Percentages larger for institutions requiring students to attend } \\
\hline Develop academic skills & 28 & 32.9 & 18 & 51.4 & 18.5 & $3.6^{*}$ & 2.2 \\
\hline Self exploration or personal development & 20 & 23.5 & 14 & 40.0 & 16.5 & 3.3 & 2.2 \\
\hline Develop writing skills & 0 & 0.0 & 1 & 2.9 & 2.9 & 2.4 & a \\
\hline Develop study skills & 34 & 40.0 & 18 & 51.4 & 11.4 & 1.3 & 1.6 \\
\hline Develop critical thinking skills & 10 & 11.8 & 6 & 17.1 & 5.3 & 0.6 & 1.6 \\
\hline Develop a connection with the institution & 36 & 42.4 & 15 & 42.9 & 0.5 & 0.2 & 1.0 \\
\hline Increase student-faculty interaction & 6 & 7.1 & 3 & 8.6 & 1.5 & 0.1 & 1.2 \\
\hline Improve second-year return rates & 12 & 14.1 & 5 & 14.3 & 0.2 & $<0.1$ & 1.0 \\
\hline Develop oral communication skills & 0 & 0.0 & 0 & 0.0 & 0.0 & a & a \\
\hline \multicolumn{8}{|l|}{ Percentages lower for institutions requiring students to attend } \\
\hline Develop support network or friendships & 12 & 14.1 & 4 & 11.4 & -2.7 & $<0.1$ & $0.8(1.3)$ \\
\hline Other & 7 & 8.2 & 2 & 5.7 & -2.5 & 0.2 & $0.7(1.5)$ \\
\hline Provide career exploration & 12 & 14.1 & 4 & 11.4 & -2.7 & 0.2 & $0.8(1.3)$ \\
\hline Develop intercultural competence & 1 & 1.2 & 0 & 0.0 & -1.2 & 0.4 & a \\
\hline Introduce the liberal arts & 1 & 1.2 & 0 & 0.0 & -1.2 & 0.4 & a \\
\hline Provide pre-professional preparation & 1 & 1.2 & 0 & 0.0 & -1.2 & 0.4 & a \\
\hline Introduce a discipline & 2 & 2.4 & 0 & 0.0 & -2.4 & 0.8 & a \\
\hline Develop information literacy & 6 & 7.1 & 1 & 2.9 & -4.2 & 0.8 & $0.4(2.6)$ \\
\hline Provide orientation to campus resources and services & 39 & 45.9 & 12 & 34.3 & -11.6 & 1.4 & $0.6(1.6)$ \\
\hline Develop financial literacy & 7 & 8.2 & 0 & 0.0 & -8.2 & 3.1 & a \\
\hline Create common first-year experience & 19 & 22.4 & 2 & 5.7 & -16.7 & $4.8^{*}$ & $0.2(4.8)$ \\
\hline
\end{tabular}

Note. Statistically significant $\chi^{\wedge} 2$ values are bolded. Odds Ratios contained within parentheses are inverted by the formula $1 / 0 R$ to improve interpretation.

${ }^{a}$ Could not be computed based on the sample size.

$* \quad p<.01$

$* * \quad p<.001$ 
Table 4

First-Year Seminar Course Objectives $(n=120)$

\begin{tabular}{|c|c|c|c|c|c|c|c|}
\hline \multirow[b]{3}{*}{ Course Topic } & \multicolumn{7}{|c|}{ Academically Underprepared Students in FYS } \\
\hline & \multicolumn{2}{|c|}{ Not Required } & \multicolumn{2}{|c|}{ Required } & \multirow[b]{2}{*}{$\%$ Diff } & \multirow[b]{2}{*}{$\chi^{2}$} & \multirow[b]{2}{*}{ OR } \\
\hline & $n$ & $\%$ & $n$ & $\%$ & & & \\
\hline \multicolumn{8}{|c|}{ Percentages larger for institutions requiring students to attend } \\
\hline Time management & 28 & 32.9 & 17 & 48.6 & 15.7 & 2.6 & 2.1 \\
\hline Academic planning & 34 & 40.0 & 19 & 54.3 & 14.3 & 2.1 & 1.8 \\
\hline Study skills & 40 & 47.1 & 20 & 57.1 & 10.0 & 1.0 & 1.5 \\
\hline Relationship issues & 7 & 8.2 & 5 & 14.3 & 6.1 & 1.0 & 1.9 \\
\hline Other & 4 & 4.7 & 3 & 8.6 & 3.9 & 0.7 & 1.9 \\
\hline Career exploration or preparation & 21 & 24.7 & 9 & 25.7 & 1.0 & 0.1 & 1.1 \\
\hline Oral communication skills & 0 & 0.0 & 0 & 0.0 & 0.0 & a & a \\
\hline Global learning & 0 & 0.0 & 0 & 0.0 & 0.0 & a & a \\
\hline \multicolumn{8}{|c|}{ Percentages lower for institutions requiring students to attend } \\
\hline Information literacy & 6 & 7.1 & 2 & 5.7 & -1.4 & 0.1 & $0.8(1.3)$ \\
\hline Critical thinking & 14 & 16.5 & 5 & 14.3 & -2.2 & 0.1 & $0.8(1.2)$ \\
\hline Health and wellness & 1 & 1.2 & 0 & 0.0 & -1.2 & 0.4 & a \\
\hline College policies and procedures & 17 & 20.0 & 9 & 14.3 & -5.7 & 0.5 & $0.7(1.5)$ \\
\hline Writing skills & 2 & 2.4 & 0 & 0.0 & -2.4 & 0.8 & a \\
\hline Specific disciplinary topic & 2 & 2.4 & 0 & 0.0 & -2.4 & 0.8 & a \\
\hline Diversity issues & 3 & 3.5 & 0 & 0.0 & -3.5 & 1.3 & a \\
\hline Campus engagement & 21 & 24.7 & 5 & 14.3 & -10.4 & 1.6 & $0.5(2.0)$ \\
\hline Campus resources & 45 & 52.9 & 14 & 40 & -12.9 & 1.6 & $0.6(1.7)$ \\
\hline Financial literacy & 8 & 9.4 & 0 & 0.0 & -9.4 & 3.5 & a \\
\hline \multicolumn{8}{|c|}{$\begin{array}{l}\text { Note. Statistically significant } \chi^{\wedge} 2 \text { values are bolded. Oc } \\
\text { formula } 1 / \text { OR to improve interpretation. } \\
\text { a Could not be computed based on the sample size. } \\
{ }^{*} \quad p<.01 \\
* * \quad p<.001\end{array}$} \\
\hline
\end{tabular}

Lastly, two-year colleges were compared on the use of high-impact practices incorporated within the first-year seminar. Again, some comparisons could not be made because of the limited number of colleges that identified the use of a particular practice. For example, only four institutions indicated that they offered undergraduate research. This was anticipated as opportunities for research are usually limited at two-year colleges although research was broadly defined as experiences for scientific inquiry, creative activities, or scholarship guided by a mentor from the faculty or research staff. Two-year colleges that required academically underprepared students to enroll in a first-year seminar were 3.9 times more likely to offer collaborative assignments and projects $\left(\chi^{\wedge} 2[1]=3.09, p=.037\right)$. There were no differences on the use of other higher impact practices. 
Table 5

First-Year Seminar High Impact Practices $(n=120)$

\begin{tabular}{|c|c|c|c|c|c|c|c|}
\hline \multirow[b]{3}{*}{ Course Topic } & \multicolumn{7}{|c|}{ Academically Underprepared Students in FYS } \\
\hline & \multicolumn{2}{|c|}{ Not Required } & \multicolumn{2}{|c|}{ Required } & \multirow[b]{2}{*}{$\%$ Diff } & \multirow[b]{2}{*}{$\chi^{2}$} & \multirow[b]{2}{*}{ OR } \\
\hline & $n$ & $\%$ & $N$ & $\%$ & & & \\
\hline \multicolumn{8}{|c|}{ Percentages larger for institutions requiring students to attend } \\
\hline Collaborative assignments \& projects & 55 & 64.7 & 29 & 82.9 & 18.2 & $3.9^{*}$ & 2.6 \\
\hline Service learning & 8 & 9.6 & 8 & 22.9 & 13.3 & 3.6 & 2.8 \\
\hline Diversity and global learning & 40 & 47.1 & 19 & 54.3 & 7.2 & 0.5 & 1.3 \\
\hline Common reading experience & 9 & 10.8 & 6 & 17.1 & 6.3 & 0.9 & 1.7 \\
\hline \multicolumn{8}{|c|}{ Percentages lower for institutions requiring students to attend } \\
\hline Writing intensive & 26 & 30.6 & 9 & 25.7 & -4.9 & 0.3 & $.8(1.3)$ \\
\hline Learning community & 26 & 31.3 & 7 & 20.0 & -11.3 & 1.6 & $6(1.8)$ \\
\hline Undergraduate research & 4 & 4.8 & 0 & 0.0 & -4.8 & 1.7 & a \\
\hline
\end{tabular}

\section{Discussion}

According to the data from this study, $70 \%$ of two-year colleges did not require academically underprepared students to enroll in a first-year seminar $(n=90)$. Among those institutions, $80 \%$ indicated that an extended orientation was the primary type of first-year seminar with a focus on creating a common first-year experience. Extended orientation seminars are reported to help to integrate students into the institution and improve retention (Permzadian \& Credé, 2016). Retention is a concern for academically underprepared institutions given that less than half of those placed into developmental courses persist to complete gateway courses (Bailey, Jeong, \& Cho, 2010). However, the retention of academically underprepared students is connected to the development of academic skills that are critical to the continual enrollment of these students. It may not be possible to achieve one aim (retention) without the other (academic performance). Extended orientation type seminars do provide information related to skill development such as time management and learning strategies, but this may not be enough to support the needs of academically underprepared students.

Among two-year colleges that required academically underprepared students to enroll in a first-year seminar, these courses were more likely to focus on basic study skills or have a hybrid component. Young and Hopp (2014) reported that "academic seminars have outpaced the growth of any other type over the past 25 years" and may reflect increased rigor in first-year curriculum (p. 49). However, the data from this study may also suggest that the type of first-year seminar is moderated by whom colleges require (or do not require) to take these courses. Institutions should design first-year seminars based on the needs of their campuses (Hunter \& Linder, 2005), but they must also be careful to consider the consequences of a common curriculum for different populations, including those who maybe academically prepared for college. Further, many students who enroll at two-year colleges intend to transfer to four-year institutions. The academic performance of these students (i.e., GPA) will be important component of admission decisions. First-year seminars that focus primarily on retention may achieve an institution's goals but may not necessarily support the long-term goals of its students.

An unanticipated finding in this study was the lack of differences in course objectives or course topics despite differences between institutions in the type of first-year seminar offered to students. Data in this study were collected from chief academic officers, chief executive officers, or chief student affairs officers and not the individual faculty teaching these seminars. Although these chief executives may be aware of general first-year seminar practices, they may be less able to speak to the specific content of first-year seminar curriculum. This may have affected the accuracy of findings. It is also possible the lack of differences in course objectives and topics may reflect uncertainty in how first-year seminars should be structured with an academic component. There is no uniform approach to the delivery of first-year seminars across institutions. If first-year seminars with an academic component are to be more effective in building the academic 
capacity of college students, then the differences in the curriculum between different seminar types will need to be better understood.

The findings from this study also bring to our attention course objectives and topics missing from the first-year seminar regardless of who was required to enroll in these courses. For example, few two-year colleges reported that developing writing skills, oral communication skills, or an introduction to a discipline was part of the first-year seminar curriculum. Perhaps these topics are embedded within others. If not, it may raise questions about why relevant topics are missing from the curriculum of an academic type first-year seminar. It is important to note that the sample size in this study was small despite the use of data from a national survey, which may have reduced the statistical power to detect differences in course objectives and topics.

Lastly, findings from this study also indicated that two-year colleges that required academically underprepared students to enroll in a first-year seminar were more likely to utilize collaborative assignments and projects in their courses. Active and collaborative learning are related to critical thinking, life-long learning, intercultural effectiveness, and socially responsible leadership (Kilgo, Sheets, \& Pascarella, 2015). The use of these practices is consistent with academically oriented first-year seminars, but other high impact practices, such as research related activities and service learning, are also related to students' academic performance (Hu, Kuh, \& Li, 2008; Kilgo et al., 2015). Consideration should be given to how these practices can be included if they help to meet academic performance goals. The primary mission of faculty at two-year colleges may not be to conduct research, but faculty can still encourage scientific inquiry and scholarship of students.

\section{Recommendations}

Two-year colleges should carefully consider students' characteristics and needs when determining the most appropriate type of first-year seminar to offer at the institution. Keup and Petschauer (2011) suggested that institutions can "fall into the trap of focusing on the students that they wish they had or used to have rather than the ones that they currently serve" (p. 18). Nationally, about $40 \%$ of community college students are placed into developmental education (Valentine, Konstantopoulos, \& Goldrick-Rab, 2017). These students are at risk in terms of both their retention and academic performance at the institution. If the retention of these students is determined to be more of a function of academic readiness, then academic type seminars may be more appropriate for these students. Yet, according to national data, extended orientation type seminars remain the primary seminar type at two-year colleges (Young \& Hopp, 2014). This may reflect a mismatch between student needs and desired student outcomes.

For those institutions that want to create a more academically oriented first-year seminar, there exist resources to support the curriculum development. The National Resource Center for The First-Year Experience and Students in Transition provides an extensive database of course syllabi and publications (e.g., Keup \& Petschauer, 2011; Groccia \& Hunter, 2012), including the University 101 Faculty Resource Manual (Friedman, Clarke, \& Strickland, 2016). Colleges may also find the Frameworks for Mathematics and Collegiate Learning course a useful resource for curriculum development (Charles A. Dana Center, 2014a). This course was developed in collaboration with the Texas Association of Community Colleges and was intended to be paired with an academic course (developmental mathematics). In contrast to other first-year seminar courses that orient students to campus resources and services, "learning frameworks courses engage students in a study of the theoretical perspectives on knowledge acquisition" (Charles A. Dana Center, 2014b, p. 2). Two-year colleges may find this resource better suited for those institutions looking to develop a more academically oriented first-year seminar.

Although tools exist to help support curriculum development, it is important to note that there is little research to indicate how varied curriculums within a given seminar type are more or less effective at achieving course aims. It is unlikely that all academic first-year seminars would result in the same level of student success. Particularly as the number of academic first-year seminars continues to grow, more information is needed to guide best practices in the implementation of this seminar type.

Two-year colleges should also consider how professional development and training is offered to first-year seminar instructors. Young and Hopp (2014) reported that adjunct faculty were more likely to be assigned to first-year seminars focused on basic study skills. Adjunct instructors at colleges are less likely to receive the same level of support and 
professional development as full-time staff and faculty. Groccia and Hunter (2012) offered suggestions for first-year seminar instructor training and development. These suggestions included consultations with individual instructors, institution-wide orientations, and institution-wide workshops. They also suggested educating first-year seminar faculty with more information about adult learning theory and "how adult learners may differ from children and adolescents" (p. 27).

\section{Conclusion}

First-year seminars support the successful transition of students into higher education. The problem is that many two-year colleges do not require academically underprepared students to enroll in these courses. This resulted in a greater likelihood that twoyear colleges offered an extended orientation type seminar when an academically oriented seminar may better support the academic performance of underprepared students, which comprise a large population of two-year college students. Colleges should consider how their existing first-year seminar curriculum meets the needs of this population.

\section{References}

Bailey, T. R., \& Alfonso, M. (2005). Paths to persistence: An analysis of research on program effectiveness at community colleges. new agenda series. Volume 6, Number 1. Lumina foundation for education. Lumina Foundation for Education. Retrieved from https://files. eric.ed.gov/fulltext/ED484239.pdf

Bailey, T., Jeong, D. W., \& Cho, S. W. (2010). Referral, enrollment, and completion in developmental education sequences in community colleges. Economics of Education Review, 29(2), 255270.

Barefoot, B. O. (1992). Helping first-year college students climb the academic ladder: Report of a national survey of freshman seminar programming in American higher education (Unpublished doctoral dissertation). College of William and Mary, Williamsburg, VA.

Blackett, K. (2008). The effects of a first-year success course on student retention and academic achievement at National American University (Doctoral dissertation). Available from ProQuest Dissertations and Theses database. (UMI No. 3318814).

Charles A. Dana Center. (2014a). Frameworks for mathematics and collegiate learning (2.0). Austin, TX: The University of Texas. Retrieved from https://dcmathpathways.org/ resources/dcmp-frameworks-mathematicsand-collegiate-learning-course-materials
Charles A. Dana Center. (2014b). Frameworks for mathematics and collegiate learning: Course essentials. Austin, TX: The University of Texas. Retrieved from https://dcmathpathways.org/ resources/dcmp-frameworks-mathematicsand-collegiate-learning-course-essentials

DeRoma, V. M., Bell, N. L., Zaremba, B. A., \& Albee, J. C. (2005). Evaluation of a college transition program for students at-risk for academic failure. Research and Teaching in Developmental Education, 21, 20-34.

Field, A. (2013). Discovering statistics using IBM SPSS statistics ( $4^{\text {th }}$ ed.). London: Sage.

Groccia, J., \& Hunter, M. S. (2012). The first-year seminar: designing, implementing, and assessing courses to support student learning and success: Volume two: Instructor training and development. Columbia, SC: University of South Carolina, National Resource Center for The First-Year Experience and Students in Transition.

Hu, S., Kuh, G. D., \& Li, S. (2008). The effects of engagement in inquiry-oriented activities on student learning and personal development. Innovative Higher Education, 33(2), 71-81.

Hunter, M. A., \& Linder, C. W. (2005). First-year seminars. In M. L. Upcraft, J. N. Gardner, B., O. Barefoot, \& Associates, Challenging and supporting the first-year student: A handbook for improving the first year of college (pp. 275-291). San Francisco: Jossey-Bass.

Karp, M. M., Raufman, J., Efthimiou, C., \& Ritze, N. (2015). Redesigning a student success course for sustained impact: Early outcomes findings, (CCRC Working Paper No. 81). New York, NY: Columbia University, Teachers College, Community College Research Center. Retrieved from https://ccrc.tc.columbia.edu/ media/k2/attachments/redesigning-studentsuccess-course-sustained-impact.pdf

Keup, J., \& Petschauer, J. W. (2011). The firstyear seminar: Designing, implementing, and assessing courses to support student learning and success: Volume One: Designing and administering the course. Columbia, SC: University of South Carolina, National Resource Center for The First-Year Experience and Students in Transition.

Kilgo, C. A., Sheets, J. K. E., \& Pascarella, E. T. (2015). The link between high-impact practices and student learning: Some longitudinal evidence. Higher Education, 69(4), 509-525.

Lang, D. (2007). The impact of a first-year experience course on the academic performance, persistence, and graduation rates of firstsemester college students at a public research university. Journal of the First-Year Experience \& Students in Transition, 19(1), 9-25. 
Levin, H. M., \& Calcagno, J. C. (2008). Remediation in the community college. Community College Review, 35, 181-207.

Osborne, J. W. (2006). Bringing balance and technical accuracy to reporting odds ratios and the results of logistic regression analyses. Practical Assessment Research \& Evaluation, 11(7). 1-6.

Padgett, R. D., Keup, J. R., \& Pascarella, E. T. (2013). The impact of first-year seminars on college students' life-long learning orientations. Journal of Student Affairs Research and Practice, 50(2), 133-151.

Pascarella, E. T., \& Terenzini, P. T. (2005). How college affects students: A Third Decade of Research. (Vol. 2). San Francisco, CA: Jossey-Bass.

Permzadian, V., \& Credé, M. (2016). Do firstyear seminars improve college grades and retention? A quantitative review of their overall effectiveness and an examination of moderators of effectiveness. Review of Educational Research, 86(1), 277-316. doi: 10.3102/0034654315584955

Porter, S. R., \& Swing, R. L. (2006). Understanding how first-year seminars affect persistence. Research in Higher Education, 47(1), 89109.

Radwin, D., Wine, J., Siegel, P., Bryan, M., (2013). 2011-12 National Postsecondary student aid study (NPSAS:12): Student financial aid estimates for 2011-12. First look. NCES 2013-165. Washington, DC: National Center for Education Statistics.

Schnell, C. A., \& Doetkott, C. D. (2003). First year seminars produce long-term impact. Journal of College Student Retention: Research, Theory \& Practice, 4(4), 377-391.

Strong American Schools. (2008). Diploma to nowhere. Washington, DC: Rockefeller Philanthropy Advisors. Retrieved from https://paworldclassmath.webs. com/8534051-Diploma-To-NowhereStrong-American-Schools-2008.pdf

Texas Higher Education Coordinating Board. (2015). 60x30TX: The 2015-2030 higher education strategicplanforTexas. Retrievedfrom http:// www.thecb.state.tx.us/reports/PDF/6664. PDF?CFID $=30926137 \&$ CFTOKEN $=87354132$

Valentine, J. C., Konstantopoulos, S., \& GoldrickRab, S. (2017). What happens to students placed into developmental education? A meta-analysis of regression discontinuity studies. Review of Educational Research, 87(4), 806-833.
What Works Clearinghouse. (2016). Strategies for postsecondary students in developmental education - A practice guide for college and university administrators, advisors, and faculty. Washington, DC: Institute for Education Sciences, What Works Clearinghouse. Retrieved from https://ies. ed.gov/ncee/wwc/Docs/PracticeGuide/ wwc_dev_ed_112916.pdf

Young, D. G., \& Hopp, J. M. (2014). 2012-2013 National survey of first-year seminars: exploring highimpact practices in the first college year. Research reports on college transitions no. 4. National resource center for the firstyear experience and students in transition. Columbia, SC: University of South Carolina, National Resource Center for The First-Year Experience and Students in Transition. 


\section{Completing College: Focus on the}

\section{Finish Line}

Hunter R. Boylan, Appalachian State University

Barbara J. Calderwood, Appalachian State University

Barbara S. Bonham, Appalachian State University

\author{
Reprinted White Paper, Copyright 2017 \\ National Center for Developmental Education \\ www.ncde.appstate.edu
}

\section{EXECUTIVE SUMMARY}

This paper contends that, although there is much to commend in the remediation reform movement, it is unlikely to attain its goals. These goals include the Lumina Foundation's target of having $60 \%$ of Americans attain a degree or certificate, the Bill and Melinda Gates Foundation's goal of doubling the number of low income students who earn a postsecondary degree, and President Obama's goal of the U.S. having the world's highest percentage of degree holders by 2020. This is due to several factors including (a) the failure to distinguish between remedial and developmental education, (b) the limited focus of reform on remedial and gateway courses, (c) the mistaken assumption that there is a causal relationship between remediation and attrition, (d) the failure to address students' reading problems, (e) the nonsystematic nature of most reform efforts, ( $f$ ) and the subsequent failure to address other causes of student attrition and the difficulties of many community college students' lives. There are, of course, many commendable efforts to improve student performance in the community college. This paper describes the most popular of these efforts. It also discusses data on their effectiveness. In spite of their success, community colleges will need to do more if they are to dramatically enhance degree and certificate completion, particularly among minority, low income, and first-generation students.

The authors suggest that there are three phases involved in attaining the dramatic increase in college completion desired by foundations and government. The first phase is to improve the quality of teaching and learning in community college classrooms. This will require a substantial faculty development effort, particularly for adjunct instructors.

The second phase is to fully integrate courses and student support services. At present, the academic and the student affairs divisions of community colleges usually operate randomly and independently of each other. Their full impact cannot be obtained unless support services are more directly linked to course goals and objectives and courses are more directly connected to the services designed to support them.

The third phase is expanding the connections between community colleges, public schools, and community services. High schools and colleges need to collaborate more closely to insure that the exit standards of secondary education are more consistent with the entry standards of postsecondary education. In addition, community colleges need to establish closer ties and better relationships with services available in the local community to address the varying nonacademic needs of our least advantaged students.

The authors then provide concrete examples of how community colleges might implement all three phases of student completion. Some of these examples represent new thinking about how community college courses and services might be organized and delivered. Many, however, represent things we already know but have, for a variety of reasons, failed to implement.

n 2013, the Lumina Foundation established the goal of having $60 \%$ of Americans attain a quality degree, certificate, or other postsecondary credential by 2025 (Lumina Foundation, 2013). The Bill and Melinda Gates Foundation's U.S. Higher Education Program set its goal to double the number of low-income students in the U.S. who earn a postsecondary degree (Bill and Melinda Gates Foundation, 2009). Meanwhile, the Obama Administration introduced the goal of having the U.S. become the world's most educated nation as measured by percent of postsecondary degree holders in the population by 2020 (The White House
Briefing Room, 2010). A combination of government and foundation funding has established or supported a number of organizations attempting to bring policy and research to bear in accomplishing these goals. It is unlikely however, that any of these goals will be met by current reform efforts.

This is not because these efforts lack federal or foundation funding. They do not lack for good ideas and good people to implement them. Nor do they lack for intelligence, effort, or integrity. Instead, they lack a broad enough focus to accomplish their goals. Reform efforts focus on a relatively small piece of the process 
of college student retention and completion while ignoring larger and equally or even more important pieces. With few exceptions they have focused on finding quick and simple solutions to the problems of student underpreparedness and low retention and graduation rates. Adherents then claim that these solutions can be successfully applied with cookie cutter regularity and minimal funding and supported by state and local policies.

The best example of such solutions are found in the movement to reform community college remediation. Most of those involved in this reform movement start off by misunderstanding the difference between remediation and developmental education. Developmental education is the integration of courses and support services guided by the principles of adult learning and development (Boylan, 1990; Saddlemire, 1978). Remediation generally refers to stand-alone courses addressing pre-college content. Unfortunately, a variety of researchers, policy makers, and news reporters use these terms interchangeably, thus confusing the issue from the outset.

It has become clear through a variety of studies that stand-alone remedial courses are often ineffective for many students (Bailey, Jeong, \& Cho, 2009; Boatman \& Long, 2010; Complete College America, 2012; Matorell \& McFarlin, 2007). This should come as no surprise. Students are typically placed in these courses using marginally accurate assessment instruments and questionable placement scores (Hughes \& Scott Clayton, 2011). Furthermore, students are typically uninformed about the consequences of placement test scores and unprepared to perform well on commonly used assessment instruments (Hodura, Smith Jaggars, Mechur Karp, 2012). Research suggests that, although more than $60 \%$ of community college students place into one or more remedial courses, fewer than a third of these students are likely to complete them (Bailey, Jeong, \& Cho, 2009).

Some organizations have seized upon this research and declared that remediation is the cause of students failing to complete college (Complete College America, 2012). Others have misunderstood what has been studied and reported that developmental education is a barrier to college completion. In fact, it is only remedial courses that have been addressed by the research, not developmental education.

The mantra that "Remediation doesn't work, we have to do something else" has often been used as an excuse to promote whatever reform is being championed by a particular organization. It has also led policy makers to erroneously believe that remediation causes attrition. Although it is a well-known fallacy to confuse correlation with causality, policy makers continue to act as though participation in remediation is the primary reason students fail to complete college. Indeed, poor remediation may be one of the many causes of student attrition, but it is far from the only one or even the major one. Others include such things as illness, finances, personal and family issues, quality of teaching, expectations, engagement, and employment (Hunt, 2013; Kuh, Kinzie, Schuh, \& Whitt, 2011; Ray, Aspland, \& Barret, 2014; Willkoxson, Cotter, \& Joy, 2011)

The belief that remediation causes attrition has led policy makers, researchers, and postsecondary education leaders to focus their reform efforts almost exclusively on reforming remedial courses, gateway courses, teaching models, or curricula. It is almost as if policy makers and leaders believe that, if remediation was eliminated or reformed, the barriers to college completion would be removed. Furthermore, they have confused remediation with developmental education and implemented policies to eliminate or reduce developmental education as well as remediation. As a result, some strong developmental programs that have contributed to student success and completion have been eliminated because of the perception that remediation and developmental education are synonymous. Having done so, policy makers in several states have targeted remediation for reform, mistakenly called it developmental education, and then claimed to have addressed the problem of college noncompletion. In fact, they have only focused on the problem of high noncompletion rates in remediation or high failure rates in gateway courses. This is consistent with their narrow view of what affects student success and completion. Ignoring a host of situational (health and family), demographic (income and ethnicity), and affective (values and attitudes) factors only exacerbates the problem.

This is not to say that the reforms fail to contribute to student success. Jobs for the Future, for instance, has done a good job of summarizing the research on some of the more popular reforms in remediation and found that many of them produce positive results for participating students (Juncos \& Collins, 2015). The Community College Research Center and other individuals and organizations have studied contemporary reform efforts and confirmed that there 
are benefits to most of them (Cho, Kopko, Jenkins, \& Jaggars, 2012; Kalamkarian, Raufman, \& Edgecombe, 2015).

Unfortunately, most of these reforms are targeted specifically toward eliminating, reforming, or redesigning community college remediation. And, as previously noted, although remediation does need to be reformed, it is developmental education that should be implemented. The plethora of other factors contributing to student attrition are generally left unaddressed, particularly when reformers see remedial courses as the sole or major cause of the problem. As a result, no matter how many reforms and innovations are introduced to remediation, high levels of student attrition will continue to occur in the nation's community colleges. This will insure that the completion goals of many private and government organizations will not be met.

\section{The Limitations of Current Reform and Innovation Efforts}

If one looks carefully at the available data on the reform of remediation, several things become clear. Most of the innovative methods proposed to improve student completion work to one degree or another. If properly implemented, just about any of the popular innovations in community college instruction will "move the needle." This is clear. Unfortunately, it is also clear that they are not always properly implemented and, even when they are, they frequently fail to move the needle far enough.

Many foundations and nonprofit organizations are advocating the bringing of promising innovations to scale. Historically, many promising innovations have had only a limited effect, mainly because they have been confined to particular programs or departments rather than being implemented throughout their host institution, because many people in postsecondary institutions are resistant to change, and because scaling efforts are expensive and time consuming (Soricone \& Pleasants McDonnell, 2016). However, considerable thought and research has gone into bringing innovations to scale in recent years (Asera, Pleasants McDonnell, \& Soricone, 2013; Public Agenda and Achieving the Dream, 2011; Soricone \& Pleasants McDonnel, 2016). This thought and research will probably contribute to improving the extent to which promising innovations are available to all students and this is positive. Unfortunately, it still may not be sufficient to bring about the dramatic changes envisioned by the Gates Foundation, the Lumina Foundation, and the federal government.

Improving mathematics pass rates from $12.3 \%$ to $62.3 \%$ (Complete College America, 2016) or improving English composition pass rates from 39\% to $75 \%$ (Cho, Kopko, Jenkins, \& Jaggars, 2012) or increasing the percentage of students who pass college-level mathematics courses to $30 \%$ or higher (Zachry Rutschow \& Diamond, 2015) represent commendable efforts. But these improvements or any others brought about by contemporary innovation will not be sufficient to meet the goals of the Lumina Foundation, the Gates Foundation, or the Obama Administration. Even if $62.3 \%$ of community college students successfully complete their first college-level mathematics class (Complete College America, 2016), they will still have to enroll in, pay for, and earn a minimum of a $C$ in at least nineteen other courses to attain an associate degree. Even if $75 \%$ of community college students successfully complete college composition (Cho, Kopko, Jenkins, \& Jaggars, 2012), they will still have to manage their adult responsibilities, respond to life crises, and maintain their motivation for however long it takes them to complete the rest of their curriculum. This is the missing link in the reform movement. Much of the legislation that results from the movement appears to be based on the flawed assumption that reforms will enable all students to be equally able to move forward; be successful in subsequent college courses; and complete a degree, certification, or diploma after participating in a particular innovation or reform. On the positive side, the Lumina Foundation (2016) and the Community College Research Center (Bailey, Smith Jaggers, \& Jenkins, 2015) have advocated for a more comprehensive approach to reform but, thus far, their work has had minimal impact on legislators.

As research points out, successfully completing the first college-level course will definitely improve the odds of a student completing college (Adelman, 2006; Boylan, Bliss, \& Bonham, 1997). But it will not improve the odds dramatically. No matter how well students do in courses targeted for reform, they will still have to run the gauntlet of challenges to their graduation. And this is where the problems lie. The overwhelming majority of contemporary reform efforts, as effective as some may be, suffer from several shortcomings.

First, they tend to focus primarily on remedial courses and gateway courses. Almost all of the most popular contemporary reform efforts measure the outcomes of their activities in terms of passing or bypassing remediation and passing initial college-level courses in English and mathematics. They do not focus on student success in post-gateway courses.

Second, reform efforts tend to focus on structural changes in courses and curriculum. They change the models used to teach remedial and gateway courses and they change the content of the courses. But they do not change the systems that these courses are part of or the rewards, expectations, or values of those systems (Bailey, Smith Jaggars, \& Jenkins, 2015).

Third, reform efforts tend to be disconnected from the rest of the institution. They often operate as 
"pet projects" of the college president or as specialized activities within a program or department. Even when they are scaled up, the scaling often involves simply serving larger numbers of students rather than creating a stronger connection between the reform and the institution (Soricone \& Pleasants McDowell, 2016).

Fourth, reform efforts tend to be random rather than systematic. They involve parts of the academic segment of the postsecondary system, but they do not engage the entire system. The reforms themselves may be systematic, but they are usually not well integrated into the larger institutional system. Course numbers, methods, and content may be changed but the Registration, Financial Aid, Academic Advising, Career Counseling, and Student Activities Offices often continue to go about "business as usual" ( Bailey, Smith Jaggars, \& Jenkins, 2015).

Fifth, reform efforts either accidentally or deliberately de-emphasize reading as a basic skill necessary for college success. Reform efforts in Florida and North Carolina, for instance, are aimed at reducing the number of students who enroll in remediation (The Florida Senate, 2013: North Carolina State Board of Community Colleges, 2014). Although this is a laudable goal, one of the methods of doing this is to integrate reading and composition courses. The result is to reduce or eliminate the number of completely reading focused courses available to students. The integration of reading and writing is a good idea and one that will benefit the majority of students (Hearn \& Snell, 2013). Unfortunately, for the weakest readers, there is no course work available to them focused specifically on developing reading skills. Yet most experts would agree that reading is a foundational skill for college success (Gray, 2014; Holschuh \& Paulson, 2013; Pugh, Pawan, \& Antommarchi, 2000). According to ACT, only 44\% of 2016 high school graduates who participated in ACT assessment were considered college ready in reading (ACT, 2016).

Finally, and perhaps most damaging, reform efforts often do not take into account those specific student characteristics contributing most to attrition. Researchers generally agree that that the following background factors have a substantial impact on attrition in college (Atwell \& Lavin, 2007; Brock, 2010; Editorial Projects in Education Research Center, 2011; Falcon, 2015):

1. Coming from a low income family,

2. Being an ethnic minority,

3. Being a first-generation student

4. Performing poorly in prior education.
Other factors also contribute to the failure of many students. In a study of over 213,000 students enrolled at public universities in the Midwest, Soria and Bultmann (2014) found that working-class students are more likely to feel alienation, isolation, and lack of belonging than middle- and upper-class students. It is likely that working class students attending community colleges experience the same feelings with subsequent impact on their performance. Based on a review of the literature, Kasworm, (2012) describes four circumstances that contribute to the attrition of adults over 25-years-old: (a) time required for college work, (b) cost of college attendance, (c) institutional policies, and d) discrimination against older students on the part of some faculty. She further points out that adult responsibilities often prevent full-time college attendance. However, there is considerable contemporary emphasis on completing college as a full-time student (Complete College America, 2011). This recent emphasis contributes to working adults' feelings of inadequacy because they simply cannot attend full-time given the other commitments in their life to work and family (Kassworm, 2012).

Only a few current reform efforts address these characteristics. Among the most effective is the Accelerated Study in Associates Programs (ASAP) of the City University of New York. This program not only provides financial incentives such as free tuition, books, and metro transportation, it also requires full-time enrollment and offers small class size, learning communities, and built-in academic, career, special programs, and personal counseling. These latter services help students address the effects of being a first-generation and/or a minority student and the financial assistance helps them overcome the effects of coming from low income backgrounds. Furthermore, the program serves its students throughout their academic careers, not just their first year (Kantor, 2011). Between 2007 when the program was introduced and 2010, participants' graduation rates were $54.9 \%$ as compared to $21.1 \%$ for a control group (City University of New York, 2011).

Another successful program that provides long-term support is the State of Washington's Integrated Basic Education and Skills (I-Best) program. This program focuses on lower skilled and English as a Second Language (ESL) students in career programs. Adult education, ESL, and career faculty at community colleges jointly design occupational courses leading to a certificate. These courses integrate basic skills and the vocabulary and expectations of a career field with 
the content of occupational courses. The program supports participating students as they progress through an occupational program by providing mentoring, tutoring, and advising for a year or more until students earn a career certificate or a degree (Zachry Rutschow \& Scheider, 2011). As in the ASAP program, students attend classes and are supported over time with mentoring and services that help them deal with the consequences of poverty and discrimination.

Perhaps the most systematic and comprehensive completion reform plan is proposed by Bailey, Smith Jaggars, and Jenkins (2015) from the Community College Research Center. These authors criticize what they refer to as the contemporary "cafeteria style self-service model" which forces students to choose courses and programs of study from a bewildering array of options and to do so with very little guidance" (p. 3). Instead, they advocate restructuring the community college curriculum to provide "guided pathways" (p. 3) that are clearly structured programs of study leading to particular certificates or degrees coupled with improved instruction integrated with support services and intensive orientation and advising. Ironically, this sounds a lot like the definition of developmental education. The guided pathways model also utilizes some of the remedial education reforms to accelerate student progress through remedial courses.

Bailey and his colleagues at the Community College Research Center are to be commended for developing a model and a set of recommendations offering a genuinely systematic and comprehensive approach to improving college completion. Unlike other reformers, Bailey, Smith Jaggars, and Jenkins (2015) address the variety of institutional and systemic failures that contribute to student attrition. Although the guided pathways model has much to recommend it, it focuses on reforming the structure of the American community college. It does not directly address the spectrum of issues that contribute heavily to the attrition of low income and minority students, although it may do so indirectly (Jenkins, Smith Jaggars, \& Bailey, 2016). Because the guided pathways model is new, few institutions have had a chance to fully implement it. As a result, there is, as yet, little research on its efficacy. However, the components of the model are well grounded in research (Jenkins, Smith Jaggars, \& Bailey, 2016).

The guided pathways model would appear to work best with full-time students: those who can commit to a course sequence and those who have the wherewithal, either through financial aid or their own resources, to regularly pay for tuition, fees, books, and supplies. But as Michael Rose points out:

I am continually struck by the hardships experienced by so many community college students. To be sure, middle class students from stable and secure backgrounds attend community college but, depending on the location of the college, many students come from low-income to destitute families; have to work 30 or more hours a week; live in cramped housing, some of which is sub-standard; are food insecure; and have health problems that are inadequately treated. For some, there are worries about immigration. Some must contend with prior involvement in the criminal justice system while others struggle with addiction. (Rose, 2016, p 2)

According to the American Association of Community Colleges, more than half of the ethnic minority students participating in postsecondary education are enrolled in community colleges. Furthermore, 58\% of community college students receive some form of financial aid, $22 \%$ of full-time community college students work full time and $40 \%$ work half time, $62 \%$ are enrolled part-time, and $36 \%$ of these students are the first in their family to attend college (American Association of Community Colleges, 2016). Given these statistics, in spite of its potential benefits, the guided pathways model will not serve all community college students, nor do its authors claim that it does (Jenkins, Smith Jaggars, \& Bailey, 2016).

Nevertheless, the ASAP Program, the I-Best Program, and the guided pathways model feature what others do not. They reorganize the curriculum and the way it is delivered for all courses, not just remedial and gateway courses. They provide support services throughout students' college careers. Their efforts are also systematic and effect entire groups of students in an organized, coherent, and purposeful manner. Unfortunately, efforts such as these are far too few. The vast majority of reforms are focused on short-term solutions that only address completing remediation and passing gateway courses.

\section{A Summary of Short-Term Reform Efforts}

To be fair, many short term reform or innovation efforts have improved student outcomes to one degree or another. Unfortunately, they do not improve outcomes sufficiently to meet the goals of foundations and government. Furthermore, they are not of sufficient duration to impact upon students as they progress past remedial and gateway courses.

\section{Accelerated and Integrated Reading and English}

Among the more successful of these innovations is that of accelerated and integrated reading and English composition originally implemented at Chabot College in California by Katie Hearn (2013). Both Jobs for the Future and the Community College Research Center support this model which involves a 1 semester, 4-hour English composition course. This course accelerates student progress by combining 2 semesters' work into a single semester (although a 2-semester option is also available to 
students). Students who complete the course are eligible to enroll in college-level English. The course not only integrates reading development with composition but also emphasizes critical thinking and familiarizing students with college-level rewards and expectations.

Based on a recent report from Jaggars, Hodura, Cho, and Xu (2016), students participating in the integrated reading and English composition course were $24 \%$ more likely to complete a college-level English course than nonparticipants. They also completed an average of 4.2 more credit hours over three years. However, both the accelerated and non-accelerated students passed the college-level English course at similar rates (Jaggars, Hodura, Cho, \& Xu, 2016).

Another popular innovation, this one championed by Complete College America, is the corequisite model of remediation. This model was originally called the Accelerated Learning Program (ALP) and developed at the Community College of Baltimore County by Peter Adams and his colleagues (Adams \& McKusick, 2014). Instead of requiring remedial courses as a prerequisite to enrollment in college-level courses, corequisite remediation provides remediation simultaneously with college-level content in a single semester.

According to Complete College America (2016), West Virginia community colleges were able to improve their pass rates in gateway mathematics courses from $14 \%$ to $62 \%$ using the corequisite model. Using the same model, they found Tennessee community colleges improved their pass rates in these courses from $12.3 \%$ to $63.3 \%$. Cho, et al. (2012) studied students enrolled in the Accelerated Learning Program at the Community College of Baltimore County between the fall of 2007 and the fall of 2011. They found that $75 \%$ of the students who participated in the ALP passed college-level English whereas only $39 \%$ of those who did not participate in the ALP passed the course.

\section{Modular Mathematics}

Modular mathematics is another model widely used in community colleges to improve student performance in developmental mathematics. This model has its origins in the personalized instruction movement of the 1970s and involves breaking the content of developmental mathematics into a series of discrete units or modules (Eyre, 2007). Students may then demonstrate mastery by taking tests covering the material in the modules. An entire course may consist of 12-16 modules and students may complete the course as fast as they are able to complete the required number of module tests, thus allowing them the opportunity for accelerated completion of developmental courses. This model was initially implemented in Virginia community college mathematics courses and later in mathematics courses in North Carolina community colleges.

The Community College Research Center and the Virginia Community College System have both issued reports on this model. Kalamkarian, Raufman, and Edgecombe (2015) found that the percentage of remedial students who completed college-level math increased from $8 \%$ to $18 \%$ in 2012. The Virginia Community College System (2014) reported that developmental mathematics success rates increased from 35\% to $40 \%$.

An innovative approach developed by Virginia Tech and promoted by the National Center for Academic Transformation is the "Emporium Model" (Twigg, 2011). This model combines computer technology that allows students to spend their time actually working mathematics problems with human assistance to answer questions when difficulties are encountered. It also utilizes a modular approach to the organization and mastery of course material. Emporium classes are usually held in large computer laboratories where instructors and/or instructional assistants circulate to help students resolve problems.

In a study conducted at Jackson State Community College, the Emporium approach contributed to raising posttest scores by $15 \%$ and increasing mathematics course pass rates by $44 \%$ (Twigg, 2011). Twigg also reported a study by the Tennessee Board of Regents indicating that students were twice as likely to earn a $\mathrm{C}$ or better in remedial math when taught using the model as students who took a traditional remedial course.

The Carnegie Foundation for the Advancement of Teaching has developed two pathways to improving student performance in remedial and college-level mathematics (Yamada \& Byrk, 2016). One pathway, Statway, integrates remedial and college-level content into a year-long course. The content includes statistical concepts and problem solving, and the methodology involves collaborative learning. The other pathway, Quantway, is a single-semester quantitative reasoning course that prepares students to succeed in a second single-semester college-level course in quantitative reasoning.

An analysis of data from 18 community colleges participating in the Statway project indicated that only $6 \%$ of students took traditional remedial mathematics and passed a college-level mathematics course in 1 year. Over a period of 4 years, an average of $48 \%$ of those participating in Statway completed both remedial and college-level courses in 1 year. Results for the Quantway project were similar. Among participating institutions, only $21 \%$ of students enrolled in traditional remedial mathematics courses passed the 
course within 1 year. An average of $56 \%$ of students participating in Quantway passed the remedial course within 1 semester (Huang, Hoang, Suleyman, \& Thorn, 2016). A further study of Statway reported that these gains were consistent regardless of gender, ethnicity, or mathematics placement scores (Yamada \& Bryk, 2016).

Uri Treisman of the Dana Center at the University of Texas-Austin, who worked with Carnegie in the development of the Statway and Quantway models, also developed the New Mathways Project. This project, implemented in cooperation with the Texas Association of Community Colleges, has developed a variety of nonalgebra based mathematics courses taught in an accelerated format. The format enables students to complete developmental mathematics and college-level mathematics within a year. These courses are also developed in line with the numeric skills required in social science, health, and liberal arts professions (Charles A. Dana Center, 2016).

Baseline data from 2011-2014 cohorts of Texas community college students indicated that only $26 \%$ of those placing into developmental mathematics courses passed them with a C or better. Of these only $20 \%$ went on to enroll in and pass a college-level mathematics courses within 3 years. In contrast, $65 \%$ of those participating in New Math Pathways developmental courses passed within 1 year and 30\% of those participating in the New Math Pathways program passed a college-level mathematics course within only 2 semesters (Zachry Rutschow \& Diamond, 2015).

All of the reform efforts described here show a positive impact on retention and completion. However, none of them represent a "magic bullet" or a panacea. All of them appear to focus on only a single phase of student success: remedial and gateway courses. Even after the reforms have been implemented and brought to scale, students will still have to negotiate the college curriculum, make wise academic and life choices, pass at least 60 hours of credit with a $\mathrm{C}$ or better, overcome language barriers, and respond to the effects of racism and poverty to attain an associate degree or transfer to a university.

\section{The Three Phases of College Success}

There are at least three phases to college retention and completion. Upon entering higher education, students must be prepared for college-level courses and successfully complete gateway courses. Retention through these initial courses is important but continued retention through graduation is equally or even more important.

\section{Phase One}

The first phase is helping students pass the courses in which they are enrolled. This is, no doubt, why so many reformers focus on remedial and gateway course completion as the object of their efforts. The first and probably easiest phase in improving college completion is getting students to successfully complete their first college courses, whether they are remedial or college level. Many reforms have focused on restructuring courses, but they ignore one of the most important components of student success: quality instruction. Quality instruction requires that instructors understand and apply adult and developmental theory and the lessons of research on teaching and learning. Developmental theory, in relation to faculty professional development, also relates to the faculty mindset of seeing students as containing a trait of college readiness versus seeing students as whole people who are in the process of developing the skills of college readiness. When faculty utter statements such as "that student is definitely not college material," they are indicating the former mindset. Developmental theory would suggest that students are not one way or another but have the capacity to develop appropriate skills given the right supports, curriculum, and pedagogical strategies. Other insidious assumptions about a person's being may lie in the trait mindset as well, such as hidden biases about ethnicity, gender, and other traits a student cannot control and which should not be used to determine readiness for college.

It is no longer sufficient for instructors to simply teach the way they have been taught. They must be able to design and deliver instruction that is effective for the adult learners who attend $21^{\text {st }}$ century community colleges, and they must do this using the most current research available. It is disappointing that few of the reforms imposed upon remediation have addressed issues such as culturally responsive teaching, inclusive instruction, adult learning theory, brainbased instruction, or cognitive psychology. Research and theory from these areas have great potential for improving the quality of instruction for all students. By and large, however, this body of knowledge has been ignored by reformers. 


\section{Phase Two}

The second phase in student retention and completion is getting the entire college to engage in systematic behaviors that contribute to student success. This involves the integration of academic and student affairs. It requires greater collaboration between student affairs professionals and academic and career faculty. Both groups should increase the time and resources devoted to enhancing students' knowledge of how to behave and succeed in college as well as supporting the development of subject matter knowledge.

As those who study college student retention consistently point out, keeping students in college is the responsibility of everyone at the institution, not just a particular course or program (Noel, Levitz, \& Saluri, 1985). There is a role to play in student completion for everyone at the college, from the grounds crew, to the cafeteria workers, to the president. But they must know what their roles are and how to play them. This, of course, requires rethinking the roles of various college personnel as well as retraining them to fulfill appropriate new roles.

\section{Phase Three}

The third phase in student retention and completion is expanding the college system to engage with secondary education as well as community educational and support systems that can be deployed to assist students to complete college. The role of high schools in preparing students for success in college is often underplayed or overlooked. High schools have the capacity for not only preparing students for college courses but also helping them understand and respond to college rewards and expectations. Unfortunately, such preparation is usually reserved only for those whose high school teachers and counselors consider them to be "college material." Basic skills and GED programs should also introduce students to nonacademic skills important to postsecondary success.

Meanwhile, the communities in which colleges are located provide a variety of services beneficial to college completion. Many community colleges lack the resources to provide a full range of services to students. However, quite a few of the services that might contribute to the success of college students, such day care or legal aid, are available in the community. Working more productively with high schools and community services is an important step in promoting college completion.

\section{Integrated Services for College Completion}

These phases of action leading to increased college completion are illustrated in Figure 1. They involve teaching and learning in the curriculum, integrating the institutional system, and collaborating with secondary education and the community. Each one of these phases makes some contribution to enhanced college completion. But it requires all three working in a systematic manner to enhance college completion to the degree that is necessary to accomplish corporate and government goals for postsecondary education (see Figure 1).

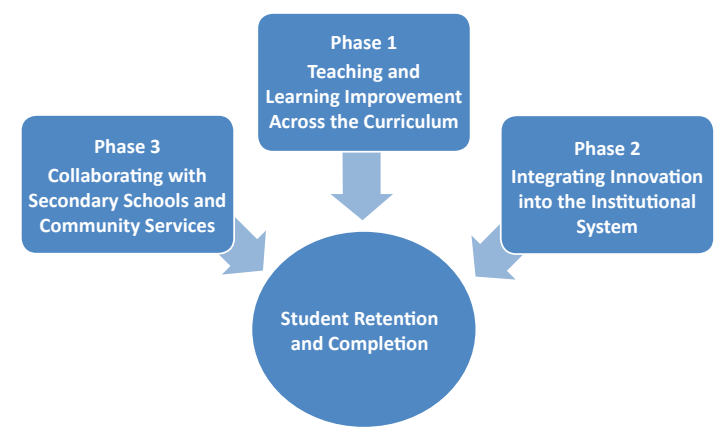

Figure 1. The three phases of action to increase college completion.

\section{Teaching and Learning Improvement}

In his classic study of community college instruction, Norton Grubb (1999) pointed out that, for institutions claiming to emphasize quality teaching, community colleges provide surprisingly little support for it. Many community colleges provide little oversight of instructors, few rewards for good teaching, and limited professional development opportunities to improve teaching. In his follow up book on basic skills instruction (Grubb, 2012), Grubb finds the situation even worse in remedial courses. It stands to reason that the weakest students would profit most from the best quality instruction. Yet the substantial majority of those teaching remedial courses are adjunct faculty (National Center for Education Statistics, 2008)., For the most part, these faculty receive little orientation to their work, little training to do it, and little support to do it right (Grubb, 2012).

This is not to say that full-time faculty are necessarily better teachers than part-time faculty. But full-time instructors at least have resources at their disposal to improve their instruction. Faculty development centers, online training programs, attendance at conferences, and participation in workshops are often provided to full-time faculty but rarely available to part-time faculty.

One of the best investments that community college leaders can make, therefore, is in professional development for adjunct faculty (Eney \& Davidson, 2006). This professional development might be made available through any number of methodologies. Examples might include common readings, interactive internet programs, on campus workshops, video conferencing, faculty inquiry groups, and mentoring by senior faculty. 
Currently, much of the professional development that takes place in the community college is more or less random. The topics of professional development activities are not always consistent nor do they necessarily reflect what instructors need to know to support student retention and completion. A primary topic for professional development should include adult development and learning theories with particular attention to lessons from research regarding cognitive neuroscience (Taylor \& Marienau, 2016). Community colleges are dealing with adult students, and yet few instructors avail themselves of the literature on adult development and learning. This is also true about research on adult teaching and learning and cognitive processing. There is a great variety of research and literature focusing on how students learn, including important work in the area of cognitive neuroscience. All community college faculty should be familiar with these bodies of literature, and these topics should form the basis of the faculty development efforts.

Training on innovative approaches to teaching and learning should also be part of this effort. Contemporary professional development activities are often focused on learning how to implement whatever innovations have been adopted by the college. A college might adopt a particular innovation and plan to bring it to scale, but the innovation will neither work well nor be brought to scale unless those participating in it are well-trained, understand the innovation, know various ways of implementing it, and have some say in how it is implemented. To the extent possible, the training should involve faculty in understanding theory supporting the models and in planning the finer points of innovation implementation. As Bailey, Smith Jaggars, and Jenkins (2015) point out, top down implementation of innovation without the meaningful involvement of instructors is generally ineffective.

Many colleges are phasing out stand-alone reading courses in favor of integrated reading and writing. This will probably help underprepared students who already read at the high school level or above. Students whose reading level is at middle school and below, however, will need substantially more reading instruction than is typically provided in an integrated reading and writing course. In fact, they will need reading instruction integrated throughout all of their courses. Training community college faculty in techniques for teaching reading is, therefore, a necessity.

Finally, faculty development efforts should focus on engaging students. There is a well-documented relationship between student engagement with faculty and student success (Center for Community College Student Engagement, 2016; Kuh, Kinzie, Schuh, \& Whitt, 2010; Quaye \& Harper, 2015). The more faculty engage in meaningful ways with students, the more successful students are likely to be in their courses and in college. Stipulating and reinforcing clear learning goals, using active learning techniques, getting students to think about their learning, being inclusive, and establishing a sense of community and safety in the classroom are examples of engagement techniques that contribute to student learning. They are also examples of topics that should be included in faculty development programs.

These topics should be the foundation for faculty development activities related to teaching today's college students, particularly those from minority, low income, or first-generation backgrounds. Institutions cannot continue to expose the weakest students to the poorest instruction and expect to improve college completion rates. Students who are most at risk are also most in need of the highest quality of teaching. Ultimately, the success of students in any course depends upon what happens in individual classrooms between individual instructors and their students. To make sure the right things happen in those classes community colleges need to:

- plan professional development activities based on assessing the needs of faculty,

- develop clearly articulated and systematic programs to meet these needs,

- provide ongoing, not episodic, faculty development activities,

- utilize a variety of methods for delivering faculty development,

- require that faculty describe what they have changed as a result of professional development

- incentivize participation in faculty development.

\section{Integrating the Institutional System}

There has been a long-standing bifurcation in postsecondary education of student affairs and academic affairs. Typically, community colleges have two different deans or vice presidents responsible for these areas. Typically, they have little to do with each other.

The student affairs staff offers their programs to whatever random group of students appears at their doorstep. The academic affairs division offers its courses to whatever random group of students enrolls. There is no effort made to see that the neediest students get the best instruction or the most support. This must change in order to attain the objectives of the college completion agenda. Student affairs professionals and academic faculty must increase their collaboration and the integration of their courses and services. Faculty must do more to inform their students about the rules and expectations of college, and student affairs professionals must do more to promote and reinforce the academic behaviors necessary for success in class. Academic advisors must 
also spend more time learning from faculty, and faculty must engage more with academic advisers. All those who interact with students should be responsible for conveying important knowledge and skills to students and encouraging the appropriate behaviors and expectations of college culture.

ACT (2015) reports that lack of college knowledge such as the information and behavioral skills necessary to for successful matriculation in academe is a major requirement for college readiness. These skills include interpersonal communication, problem solving, help seeking, task management, study strategies, and personal decision making. They also include informational knowledge such as how to obtain financial aid, register for classes, calculate a grade point average, get career development assistance, find tutors, or seek advice and counseling. As early as 1980, Boylan (p.11), called these "academic management" skills and argued that underprepared students generally did not begin college with them. He also suggested that such students were in danger of attrition unless they developed these skills early in their college careers.

Student affairs professionals deal with the enhancement of these skills and knowledge on a regular basis, yet many students are totally unaware of the assistance these professionals can provide. It is well known among student support professionals that the students most in need of their services are least likely to voluntarily participate in them (Collins \& Simms, 2006; Winograd \& Rust, 2014). Meanwhile, instructors tend to focus on content and process skills in their subject areas and view their roles entirely in terms of their discipline. They, too, should be promoting participation in support services and working to integrate what they do with the work of advisors, counselors, financial aid officers, and career development specialists. Integrating the efforts of both sets of professionals is essential to improving college completion.

Such integration requires that instructors and student affairs professionals work together to find ways of communicating college knowledge to students. They must also work together to promote students' personal and behavioral development. Resulting integration efforts might involve student affairs professionals visiting classes, or it might involve training faculty to communicate essential college knowledge and promote student development. It might involve student affairs professionals in teaching college knowledge and appropriate behaviors during class meetings. It might involve greater faculty participation in on-going orientation or the revision of syllabi to include problems and activities that help students develop college knowledge and appropriate academic behaviors. Faculty can help explain the rewards and expectations of academe during orientation or talk about them during class. Writing faculty can encour- age students to do research on careers of interest or to discuss their reasons for being in college. Mathematics faculty can link math problems to careers or use them to promote practical problem solving skills.

In addition to integrating academic and student affairs, colleges must also integrate the concept of completion into the culture and behavior of the college and its faculty and staff. First, it is important for college leaders to explain and support the notion that everyone from the cafeteria worker to the president is responsible for student completion. It is also necessary to provide the training required for faculty and staff to promote student completion. Furthermore, college leaders will need to find ways to incentivize the faculty and staff behaviors that will lead to supporting the completion agenda. Everyone on campus can contribute to student completion by:

- providing a consistently welcoming environment for students;

- creating a safe environment for students by refusing to tolerate bigotry, discrimination, shaming, or bullying in any college facility or activity;

- promoting a sense of community among students, faculty, and staff;

- making tutoring, counseling, advising, financial aid, and student activities readily and aggressively available to students;

- encouraging faculty and staff involvement in student and campus activities

- celebrating student success whether it be attaining a GED or graduating with a BA or AA degree.

Some institutions of higher education focus on improving "on-ramps and off-ramps" for students to more easily get back on their educational track after interruptions caused by life events outside their control. The Nevada Governor's Office of Economic Development mapped out an example of such programming (Heise, 2016), and MDRC describes general core elements shared by programs with this design. (Kruglaya \& Kazis, 2016). Also, Minority-Serving Institutions have a long tradition of serving diverse students and offer tactics Predominantly White Institutions might follow to improve supports for underrepresented student success.

\section{Collaboration with the Community and Secondary Education}

In 1986, Ernest Boyer, then President of the Carnegie Foundation, claimed that "One of our most disturbing findings is the discontinuity that exists between public schools and institutions of higher learning" (p. 254). Unfortunately, this observation is still true in many respects. The Center for Community College Student Engagement (2016) points out that most students leave high school believing they are fully prepared for college and are surprised to be placed in 
remedial courses. Many of these students completed their high school courses with $A$ and $B$ grades but are still considered undereprepared for college. The gap between expectations and reality for high school graduates is largely the result of the secondary and postsecondary segments of education failing to communicate adequately with each other (Boyer, 1986).

The academic requirements and expectations of college are different than those of high school. Generally, high school teachers are considered responsible for student learning. In college, students are responsible for their own learning. Yet few students understand this upon entry, particularly first-generation college students. Furthermore, there is often a mismatch between the subject matter as taught in high school and as taught in college (Glancy, Dounay Zinth, Anderson, Millard, \& Fulton, 2014). The difference in academic requirements between high school and college need to be identified explicitly and conveyed to students consistently. For instance, giving tests over the common core and college requirements during the junior year of high school is a good idea. But it must also be accompanied by counseling on the meaning of placement test scores, their importance for entering college students, and the ways in which they are used by colleges.

Many other students are underprepared for college because they lack knowledge of postsecondary behavioral expectations or of the processes involved in matriculation through college. The National Association of Student Financial Aid Administrators (2016) reports that low income students typically overestimate the costs of college and underestimate their capacity for meeting these costs. Bailey, Smith Jaggars, and Jenkins (2015) argue that a large number of incoming community college students are underprepared to choose a program of study or a career.

Students' lack of knowledge in these areas leads to increasing the contact load of college-level student affairs professionals who must work with students in academic advising and career counseling. It also leads to annoyance among faculty when students appear to be ignorant of the role of college or their purpose for being there. Furthermore, it leads to frustration among students who fail to understand that their own actions or inactions are causing many of the problems they face in matriculation. The contact load and the frustrations and annoyance could be eased if there was greater communication between high school personnel and academic and student affairs professionals at local colleges and expanded efforts to teach high school students about college rules, expectations, and procedures.

But it is difficult for high school teachers and staff to help prepare students for the academic and nonacademic demands of college if no one from the postsecondary sector talks with them about these demands. High school and college advising and support service professionals need to communicate on a regular and systematic basis. They need to discuss the affective and behavioral expectations of students and the rewards and expectations of colleges. In the process, they need to discover ways of communicating this information more systematically to students. Guidance counselors and college academic advisers not only need to work with each other, they also need to work with high school teachers to help communicate important information about college expectations, processes, and rewards.

Today's college students face a variety of life problems and issues. A recent report by Wood, Harris, and Delgado, (2016) found that, among California community college students, one third express housing insecurity and $12 \%$ experience food insecurity. The American Association of Community Colleges (2016) also reports that $44 \%$ of community college students work part time and $22 \%$ work full time. This often results in conflicts between the requirements of work and the requirements of college attendance.

Collaboration between agencies in the service of college completion should not be limited to public schools and colleges. In any given community there are resources for helping community members find support, services, health care, and legal advice. There are homeless shelters, prenatal care counselors for expectant mothers, community mental health agencies, legal aid societies, public health benefits for the poor, and shelters for abused spouses. Yet community college personnel rarely work with these agencies and, if they do refer students, may do so randomly.

Students with legal problems may be referred to the local Legal Aid Society by a faculty member but only if that faculty member knows of the agency or has worked with it. There are community agencies that can assist students in dealing with housing insecurity but are unlikely to do so unless someone at the college makes a referral. Community colleges need to work collaboratively with community agencies to be sure that students in need have support in dealing with life and work contingencies. In doing so, colleges should:

- take inventory of the available services in their community;

- designate individuals to establish regular contact and communication with these agencies;

- establish regular contact with community agencies that might provide support to students;

- provide something to community agencies, such as facility usage or consultation, in return for their services;

- provide general and contact information on these services to all faculty and staff who work with students

- provide training to faculty and staff on how to make referrals to these services. 


\section{Conclusion}

These ideas and others like them have been part of the conversation about postsecondary improvement for years. Some institutions have instituted many of them. Nevertheless, the systematic, institutional actions required to dramatically move the needle forward on college completion are the exception rather than the rule. One of the major reasons for this is that quality reform, innovation, and institutional change all take time, training, and money. Implementing the three phases of college completion--teaching and learning improvement across the curriculum, integrating innovation into the institutional system, and collaborating with secondary schools and community services--will not come easily. To implement them faculty, staff, and administrators will have to put in time: time to meet, time to plan, time to collaborate, and time to truly implement innovation and conduct formative evaluation of it. Unfortunately, time is a very scarce resource in American community colleges. Adapting to new technology, meeting state and federal compliance regulations, having fewer personnel resources and teaching larger classes because of cutbacks in postsecondary funding, and a host of other factors have all contributed to usurping the time available for college faculty and staff. Yet the changes required to truly expand college completion are going to take up large amounts of that very valuable time, and someone will have to pay for it.

There is a statement often seen in auto repair shops that says "I can do it fast, I can do it cheap, and I can do it well. Please select two of the above." This reality adequately states the conundrum faced by community colleges. They can implement change quickly, they can do it cheaply, or they can implement it with quality but they can only do two at once, Meanwhile, they are being called upon by legislators and state system offices to do all three at once (see Figure 2). Instead, what happens at many institutions is that community colleges do manage to implement change quickly and cheaply. Unfortunately, they are unable to provide the faculty and staff training, the support services personnel, or the financial incentives either to provide the high quality of innovation or to sustain that quality. This is the reality in states that mandate change without providing adequate time or resources. It is well past time to confront legislators and state system officers with this reality. If policy makers truly desire quality implementation of reform, they will have to provide either the time or the money necessary to attain it. In most states, community college budgets have already been stretched to the limit with subsequent challenges to quality (Boggs, 2004; Jenkins \& Belfield, 2014). Budget tightening also has the effect of reducing the amount of time available for faculty to initiate, engage, and support innovation, as fewer faculty and staff have to do more work. It is unlikely that the kind of changes that need to take place to truly move the bar on college completion can take place in such a fiscal environment. If legislators and policy makers want change and improvement, they will have to pay for it.

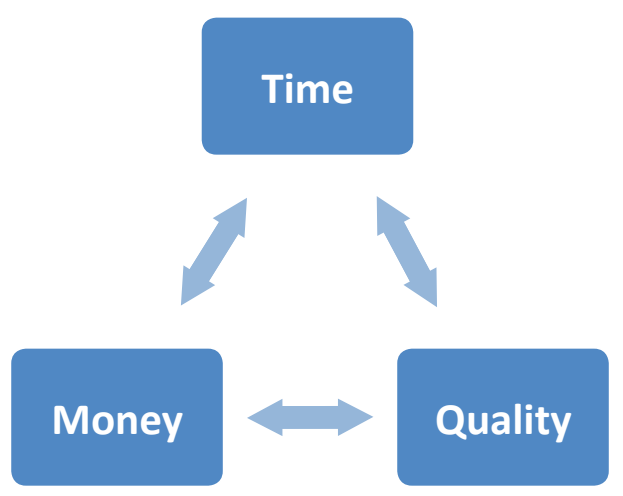

Figure 2 . The time, money, and quality conundrum.

The bottom line is that none of the ideas proposed here will take place quickly or easily. Some of those in the reform movement have promised quick results for a small investment of time and money. Others have stated that their reforms will be costly, at least at the outset, and will take a considerable amount of time to implement. The claims of the latter are likely to be more accurate. Even those claims, however, are probably optimistic unless efforts are refocused in the reform and completion agenda. This refocus should address the major student characteristics that contribute to student attrition such as being an ethnic minority, coming from a lower socioeconomic background, being a first-generation college student, or having a history of academic failure. It should address faculty training and development with particular attention to teaching reading. It should address all three phases in the college completion process, not just remediation or gateway courses. In doing so, it should also address the fact that college students are developing adults with a wide range of strengths and weaknesses; positive and negative life circumstances, advantages and disadvantages; attitudes and values; backgrounds and cultures; and hopes, fears, and frustrations. To be successful in meaningfully expanding college completion, all the players in postsecondary education, from clerks to faculty to administrators to politicians, must do a much better job of responding to all of these, not just student performance in remedial and gateway courses. Furthermore, legislators and higher education leaders must do a better job of providing time and money for quality change. Improving college completion rates, particularly for low income, minority, and first-generation students is a long distance race. It will require everyone in the race to focus on the finish line, not just first hundred meters. 


\section{References}

ACT. (2016). The condition of college and career readiness 2016. lowa City, IA: Author.

ACT. (2015). The condition of college and career readiness 2015. lowa City, IA: Author.

Adams, P., \& McKusick, D. (2014). Steps and missteps: Redesigning, piloting, and scaling a developmental writing program. New Directions for Community Colleges, 2014 (167), 15-25.

Adelman, C. (2006). The tool box revisited: Paths to degree completion from high school through college. Washington, DC: U.S. Department of Education.

American Association of Community Colleges. (2016). AACC 2016 fact sheet. Retrieved from http://www.aacc.nche.edu/AboutCC/ Documents/AACCFactSheetsR2.pdf

Asera, R., Pleasants McDonnell, R., \& Soricone, L. (2013, July). Thinking big: A framework for states on scaling up community college innovation. Boston, MA: Jobs for the Future.

Attewell, P., \& Lavin, D. (2007). Passing the torch: Does higher education for the disadvantaged pay off across the generations. New York, NY: Russell Sage Foundation.

Bailey, T., Jeong, D. W., \& \& Cho, S. W. (2009). Referral, enrollment, and completion in developmental education sequences in community colleges. New York, NY: Community College Research Center.

Bailey, T., Smith Jaggars, S., \& Jenkins, D. (2015). Redesigning America's community colleges: A clearer path to student success. Cambridge, MA: Harvard University Press.

Bill and Melinda Gates Foundation. (2009). New initiative to double the number of low-income students in the U.S. who earn a postsecondary degree. Retrieved from http://www. gatesfoundation.org/Media-Center/PressReleases/2008/12/New-Initiative-to-Doublethe-Number-of-Lowlncome-Students-in-theUS-Who-Earn-a-Postsecondary-Degree

Boatman, A., \& Long, B. (2010). Does remediation work forallstudents: How the effects ofpostsecondary remedial and developmental courses vary by level of academic preparation. New York, NY: National Center for Postsecondary Research.

Boggs, G. (2004). Community colleges in the perfect storm. Change, 36(6), 6-11.

Boylan, H. (1980). Academic intervention in developmental education. Journal of Developmental \& Remedial Education, 3(3), 10-12.

Boylan, H. (1990, July). Developmental education 101. Paper presented at the Kellogg Institute for the training and certification of developmental educators, Boone, NC.
Boylan, H., Bliss, L., \& Bonham, B. (1997). Program components and their relationship to student performance, Journal of Developmental Education, 20(3), 2-8.

Boyer, E. (1986). Smoothing the transition from school to college. Phi Delta Kappan, 68(4), 283-287.

Brock, T. (2010). Young adults and higher education: Barriers and breakthroughs to success. The Future of Children, 20(1), 109-132.

Center for Community College Student Engagement. (2016, July). Expectations meet reality: The underprepared student at community colleges. Austin, TX: Author.

Cho, S., Kopko, E., Jenkins, D., \& Jaggars, S. S. (2012). New evidence of success for community college remedial English students: Tracking the outcomes of students in the accelerated learning program (ALP) (CCRC Working Paper Number 53). New York, NY: Community College Research Center, Teachers College, Columbia University.

City University of New York. (2011). Proposals to improve success rates for students in developmental education at CUNY. New York, NY: CUNY Office of Academic Affairs. Retrieved from http://www2.cuny.edu/wp-content/ uploads/sites/4/media-assets/Report-of-theRemediation-Working-Group.pdf

Collins, W., \& Sims, B. C. (2006). Help seeking in higher education academic support services. In S.A. Karabenick \& R.S. Newman (Eds.), Help seeking in academic settings: Goals, groups, and contacts (pp. 202-223). Mahwah, NJ: Lawrence Erlbaum.

Complete College America. (2011, September). Time is the enemy. Indianapolis, IN: Author.

Complete College America. (2012). Remediation: Higher education's bridge to nowhere. Indianapolis, IN: Author.

Complete College America. (April, 2016). Co-requisite remediation: Spanning the completion divide. Retrieved from http://completecollege.org/ spanningthedivide/

The Charles A. Dana Center. (2016). New mathways project curricular materials. Retrieved from http://www.utdanacenter.org/highereducation/new-mathways-project/newmathways-project-curricular-materials/

Editorial Projects in Education Research Center. (2011, July 7). Issues A-z: Achievement gap. Education Week. Retrieved from http://www.edweek. org/ew/issues/achievement-gap/

Eney, P., \& Davidson, E. (2006). Improving supervision of part-time instructors. Journal of Developmental Education, 30(1), 2-11. 
Eyre, H. E. (2007). Keller's personalized system of instruction: Was it a fleeting fancy or is there a revival on the horizon. Behavior Analyst Today, 8(3), 317-324.

Falcon, L. (2015, June). Breaking down barriers: First generation college students and college success. Innovation Showcase. Retrieved from www.league.org/innovation-showcase/ breaking-down-barriers-first-generationcollege-student-and.college-success

Glancy, E., Dounay Zinth, J., Anderson, L., Millard, M., \& Fulton, M. (2014, October). Blueprint for college readiness: A 50-state policy analysis. Denver, CO: Education Commission of the States.

Grubb, W. N. (1999). Honored but invisible: An inside look at community college teaching. New York, NY: Routledge.

Gray, W. S. (2014). Reading difficulties in college: The natureand extent of reading deficiencies among college students. In S. Armstrong, N. Stahl, \& H. Boylan (Eds.), Teaching developmental reading (106-113). Boston, MA: Bedford/St. Martins.

Grubb, W. N. (2012). Basic skills instruction in community colleges: Inside and outside classrooms. New York, NY: Routledge.

Hearn, K., with Snell, N. (2013). Toward a vision of accelerated curriculum and pedagogy: High challenge, high support classrooms for underprepared students. Oakland, CA: Learning Works.

Heise, K. (2016, October 21). LEAP framework. Retrieved from http://system.nevada.edu/ tasks/sites/Nshe/assets/File/BoardOfRegents/ Agendas/2016/oct-mtgs/bor-joint-refs/BOR3a.pdf

Hodura, M., Smith Jaggars, S., \& Mechur Karp, M. (2012, November). Improving developmental education placement and assessment: Lessons from Community colleges across the country. (CCRC Working Paper Number 51). New York, NY: Community College Research Center, Teachers College, Columbia University.

Holschuh, J. P., \& Paulson, E. J. (2013). The terrain of developmental reading. Oak Creek, WI: College Reading and Learning Association.

Huang, M., Hoang, H., Suleyman, Y., \& Thorn, C. (2016). Community college pathways: Impact report 2014-2015. Stanford, CA: Carnegie Foundation for the Advancement of Teaching.

Hughes, K., \& Scott Clayton, J. (2011). Assessing developmental assessment in community colleges (CCRC Working Paper No. 19). New York, NY: Community College Research Center, Teachers College, Columbia University.
Hunt, P. F. (2012, September/October). Why some students leave college during their senior year. Journal of College Student Development, 53, 737-742.

Jaggars, S. S., Hodara, M., Cho, S.W., \& Xu, D. (2016). Three accelerated developmental education programs: Features, student outcomes, and implications. Community College Review, 43(1), 3-26.

Jenkins, D., \& Belfield, C. (2014). Can community colleges continue to do more with less? Change, 46(3), 6-13.

Jenkins, D., Smith Jaggars, S., \& Bailey, T. (2016, July 18). Lessons learned about guided pathways, Inside Higher Education. Retrieved from https:// www.insidehighered.com/views/2016/07/18/ what-were-learning-about-challengesimplementing-guided-pathway-reforms-essay

Juncos, A., \& Collins, L. M. (2015). Literature review: Models for developmental education redesign. Boston, MA: Jobs for the Future.

Kalamkarian, H. S., Raufman, J., \& Edgecombe, N. (2015). Statewide developmental education reform: Early implementation in Virginia and North Carolina. New York, NY: Community College Research Center, Teachers College, Columbia University.

Kantor, M. J. (2011). American higher education: First in the world. Change, 43(3), 7-19.

Kassworm, C. E. (2012). U.S. adult education: One context of lifelong learning. International Journal of Continuing and Lifelong Learning, 5(1), 1-9.

Kuh, G., Kinzie, J., Schuh, J., \& Whitt, E. (2010). Student success in college: Creating conditions that matter. San Franciso, CA: Jossey-Bass.

Kruglaya, I., \& Kazis, R. (2016, March 9). MDRC research on Career Pathways [Text]. Retrieved from http://www.mdrc.org/publication/mdrcresearch-career-pathways

Lumina Foundation. (2013, February). Lumina Foundation strategic plan: 2013 to 2016. Indianapolis, IN: Author. Retrieved from http://www.luminafoundation.org/files/ resources/2013lumina-strategic-plan.pdf

Lumina Foundation. (2016, October). Lumina Foundation strategic plan for 2017 to 2020. Indianapolis, IN: Author. Retrieved from https:// www.luminafoundation.org/files/resources/ lumina-strategic-plan-2017-to-2020.pdf

Matorell, P., \& McFarlin, I. (2007). Help our hindrance: The effect of college remediation on academic and labor market outcomes. Santa Monica, CA: RAND Corporation. 
National Association of Student Financial Aid Administrators. (2016, July). Mapping critical student decisions through college: Reviewing the literature and landscape through a behavioral lens. Washington, DC: U.S. Department of Education.

Noel, L., Levitz, R., \& Saluri, D. (1985). Increasing student retention. San Francisco, CA: JosseyBass.

North Carolina State Board of Community Colleges. (2014, March 21). Multiple measures of placement. Retrieved from http://www. nccommunitycolleges.edu/sites/default/files/ state-board/program/prog_04_multiple_ measures_2-12-15.pdf

Public Agenda and Achieving the Dream. (2011). Scaling community college innovations. New York, NY: Public Agenda.

Pugh, S. L., Pawan, F., \& Antommarchi, C. (2000). Academic literacy and the new college learner. In R. Flippo \& D. Caverly (Eds.) Handbook of college reading and study strategy research. (25-42). Mawhwah, NJ: Lawrence Erlbaum Associates.

Quaye, S., \& Harper, S. (2015). Student engagement in higher education: Theoretical perspectives and practical approaches for diverse populations. New York, NY: Routledge.

Ray, J., Aspland, J., \& Barret, D. (2014). Choosing to stay: Looking at retention from a different perspective, Studies in Higher Education, 39(9), 1700-1714.

Rogers, C. R. (1961). On becoming a person: $A$ therapist's view of psychotherapy. New York, NY: Houghton-Mifflin.

Rose, M. (2016, June). Reassessing a redesign of community colleges, Inside Higher Education.

Retrieved from https://www.insidehighered. com/views/2016/06/23/essay-challengesfacing-guided-pathways-model-restructuringtwo-year-colleges

Saddlemire, G. (1978, March). Reflections on the developmental education movement. Paper presented at the American College Personnel Association Convention, Detroit, MI.

Soria, K., \& Bultmann, M. (2014). Supporting working class students in higher education. NACADA Journal, 34(2), 51-62.

Soricone, L., \& Pleasants McDonnell, R. (2016). Scaling innovation in community colleges: A guide to action. Boston, MA: Jobs for the Future.

Taylor, K., \& Marienau, C. (2016). Facilitating learning with the adult brain in mind. San Francisco, CA: Jossey-Bass.

The Florida Senate. (2013, July 1) CS/CS/SB 1720 education. Retrievedfromhttp://www.flsenate. gov/Committees/BillSummaries/2013/ $\mathrm{html} / 501$
Twigg, C. (2011). The Emporium Model: Higher education's silver bullet. Change, 43(3), 25-34.

The White House Briefing Room. (March, 2010). Higher education. Retrieved from https:// www.whitehouse.gov/issues/education/ higher-education

Virginia Community College System. (2014). Initial review of the impact of developmental education redesign at Virginia's community colleges. Richmond, VA: Virginia Community College System.

Willcoxon, L., Cotter, J., \& Joy, S. (2011). The impact on attrition of student experiences throughout undergraduate degree studies in six diverse universities, Studies in Higher Education, 36(3), 331-352.

Winograd, G., Rust, J. P. (2014). Stigma, awareness of support services, and academic help-seeking among historically underrepresented first year college students. The Learning Assistance Review, 19(2), 17-41.

Wood, J. L., Harris, III F. \& Delgado, N. R. (2016). Struggling to survive - Striding to succeed: Food and housing insecurities in the community college. San Diego, CA: Community College Equity Assessment Lab.

Yamada, H., \& Byrk, A. S. (2016). Assessing the first two years' effectiveness of Statway: A multilevel model with propensity score matching. Community College Review, 44(3), 179-204.

Zachry Rutschow, E., \& Schneider, E. (2011). Unlocking the gate: What we know about improving developmental education. New York, NY: MDRC.

Zachry Rutschow, E., \& Diamond, J. (2015). Laying the foundations: Early findings from the new mathways project. New York, NY: MDRC. 


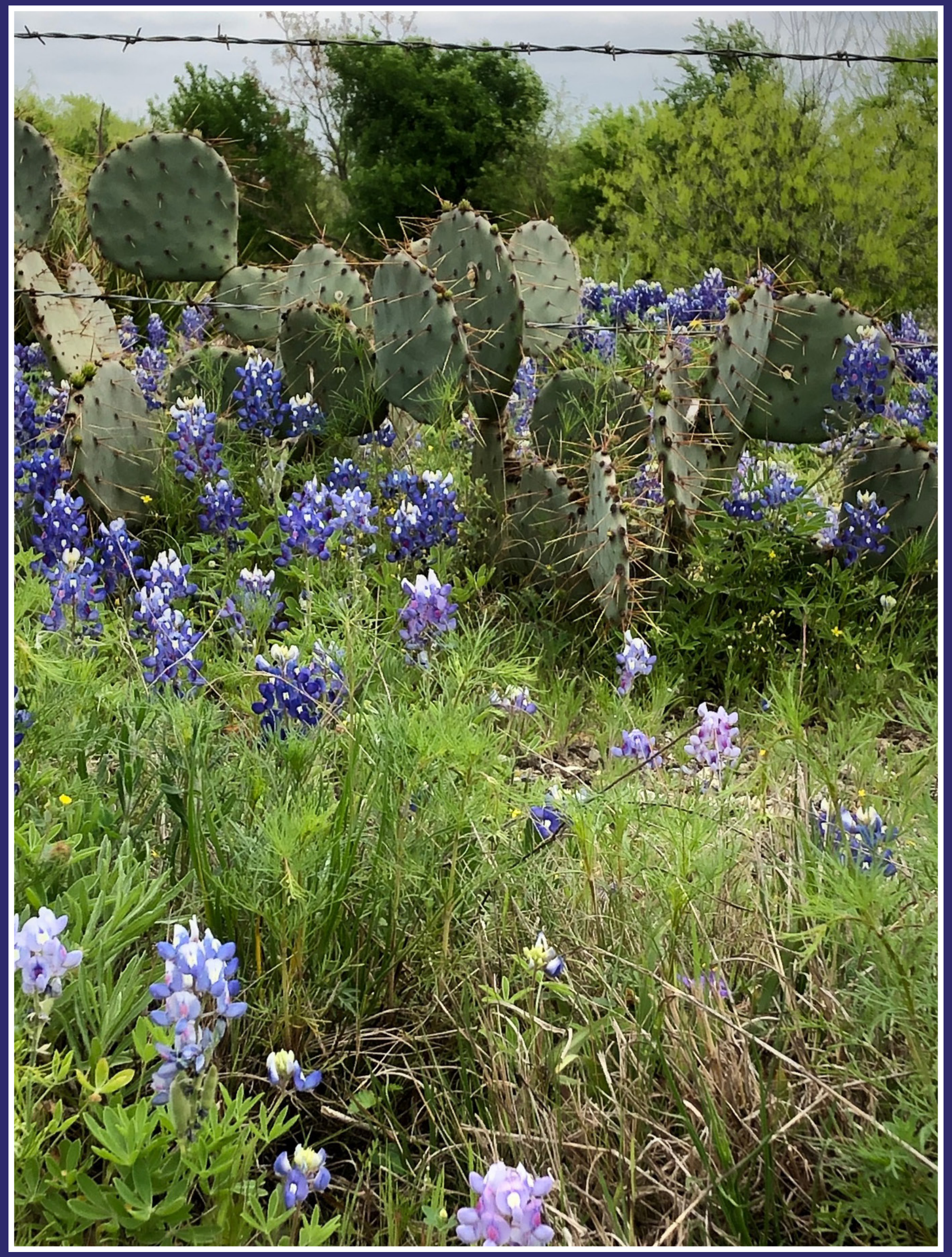




\section{SI or Peer Tutoring: Is One Really Better Than the Other?}

\section{Klara Keim \\ Michelle Kiser}

\section{ABOUT THE AUTHORS}

Klara Keim is a senior at Texas Tech University, majoring in microbiology. She recently completed an internship at the National Institute of Standards and Technology (NIST) and is planning on continuing research through graduate studies.

Michelle Kiser, Ed.D., is Senior Director of the Texas Tech University Support Operations for Academic Retention (SOAR).

et's say a fictional college student, Charlie, is taking a difficult course, such as chemistry. Charlie's first test did not go as well as expected, and Charlie needs help with not only the subject material, but also with how to study in general. This student is motivated to get help with chemistry and finds that the school offers free peer tutoring (Topping, 1996) and supplemental instruction (SI) (Arendale, 1994). Which should Charlie choose? I am both a chemistry peer tutor and an SI leader at Texas Tech University, and I face this challenge on a weekly basis because I provide both services for students. Peer tutoring and SI are both vastly different in methodology and uses, which leaves me to question which service is of greatest benefit for Charlie and other students seeking help in chemistry.

The SI program at Texas Tech University offers peer-led review sessions by undergraduate students who have made A's in the class previously and who show strength in the course material. SI sessions are hosted twice a week. The SI leader attends the class alongside enrolled students and then creates a packet of review material. As an SI leader, I have the independence to make my own review packets and supplemental items for the course under the supervision of the SI administrator. This is unique because I get to choose what I think would be the most helpful for students to focus on for test preparatory purposes. Often I focus on the content that will most likely be tested and that also presents the greatest challenge to students, which helps them increase the likelihood of success on their tests.
As for peer tutoring, Texas Tech University offers a wide variety of tutoring options varying in the target students. For example, there is Honors College tutoring for honors students, Resident Housing Association tutoring offered to students living in specific residence halls, and the Marsha Sharp Center tutoring available to student athletes. Personally, I am employed at the Texas Tech University Learning Center, where we provide free open-access tutoring. Students can drop in according to their schedules and needs. The tutoring resources we provide are unique in that we provide tutoring for anyone who is a student at Texas Tech University. A peer tutoring session typically entails the student sitting down individually with their tutor and working to solve homework problems and ask questions about specific topics that they are struggling with.

Supplemental Instruction benefits students at Texas Tech University in many ways. Students can review the material covered throughout the week on a more personalized and peer-to-peer level. Since a student is leading the session, a relaxed environment is generated that is open to discussion and questions (Altomare \& Moreno-Gongora, 2018). Students seek out SI because they did not understand something in the classroom, and they need further explanation. During my sessions, I try to act as a facilitator of material between the professor and student. I strategically organize my packets and re-teach the material to promote student success on exams. The amount of material students cover in a week is immense, and I try to help them sort out exactly what are key elements, what is going to be tested, and how it will be tested.

Peer-tutoring relies on completely different strategies than SI. During peer-tutoring, students typically come in for help because they need oneon-one help with getting through their homework (Clarence, 2016). I provide less test preparation and more homework help when I am tutoring. With SI, students who attend sessions consistently from the beginning of the semester are set up for success because they are taking control of learning the material

\section{In an ideal world, I think that}

\section{Sl and tutoring would be} most effective in conjunction with one another. 
from the start. These students typically have a base understanding of the material. Tutoring seems to me more like a catch-up session for students. They express a feeling of being lost, possibly because they are not attending class or SI sessions, so they do not have the base knowledge for solving their homework. My job is to guide them back to understanding. On the other hand, SI can oftentimes lead students to depend heavily on the instructor to help them develop a step-by-step method to solve the problem in addition to receiving the answer. Therefore, to benefit fully from attending $\mathrm{SI}$, students must practice problems independently and utilize the instructor as a means to test their knowledge.

In an ideal world, I think that SI and tutoring would be most effective in conjunction with one another. Students would attend class as scheduled then attend SI for a break-down review of the material that week. I think that then, if they do not understand, they should attend peer tutoring for further clarification of specific concepts. Returning to the original question regarding which is better-SI or peer tutoring-it turns out that you cannot ask which is better because effectiveness depends on the details: the student, how much they have attended class, and the context (e.g. does the student need help with a single assignment or more extensive assistance?).

I have tried to take a balanced view in my discussion, but I must confess that I personally prefer $\mathrm{SI}$. The vibe that I get from $\mathrm{SI}$ is more open, relaxed, and an altogether fun way to learn a really difficult subject. SI gives students a chance to interact and learn in a less formal environment that further motivates them to receive extra help, check their knowledge, and improve their grades (Marrone \& Draganov, 2017). Supplemental Instruction feels more like a preventative measure in that, if students utilize it first, they might not need peer tutoring later on. UItimately, neither option should be considered better nor worse but should be considered in terms of how the student uses it to achieve academic success. In this case, I believe that it would benefit Charlie to utilize both $\mathrm{SI}$ and peer tutoring resources. He could maximize his chances of understanding the material by attending SI sessions regularly to gain a deeper understanding of the material taught in class. Then, as further questions arise while solving homework problems and studying, he should seek more individualized help by meeting with a peer tutor.

\section{References}

Altomare, T. K., \& Moreno-Gongora, A. N. (2018). The role and impact of Supplemental Instruction in accelerated developmental math courses. Journal of College Academic Support Programs, 1(1), 19-24.

Arendale, D. R. (1994). Understanding the Supplemental Instruction model. In D. C. Martin and D. R. Arendale (Eds.), Supplemental Instruction: Increasing student achievement and retention. (New Directions in Teaching and Learning, No. 60, pp. 11-21). San Francisco, CA: Jossey-Bass.

Clarence, S. (2016). Peer tutors as learning and teaching partners: A cumulative approach to building peer tutoring capacity in higher education. Critical Studies in Teaching and Learning, 4(1), 39-54.

Marrone, M., \& Draganov, L. (2017). Peer Assisted Learning: Strategies to increase student attendance and student success in accounting. In L. Wood and Y. Breyer (Eds.), Success in Higher Education (pp. 149-165). Singapore: Springer.

Topping, K. J. (1996). The effectiveness of peer tutoring in further and higher education: A typology and review of the literature. Higher Education, 32(3), 321-345. 

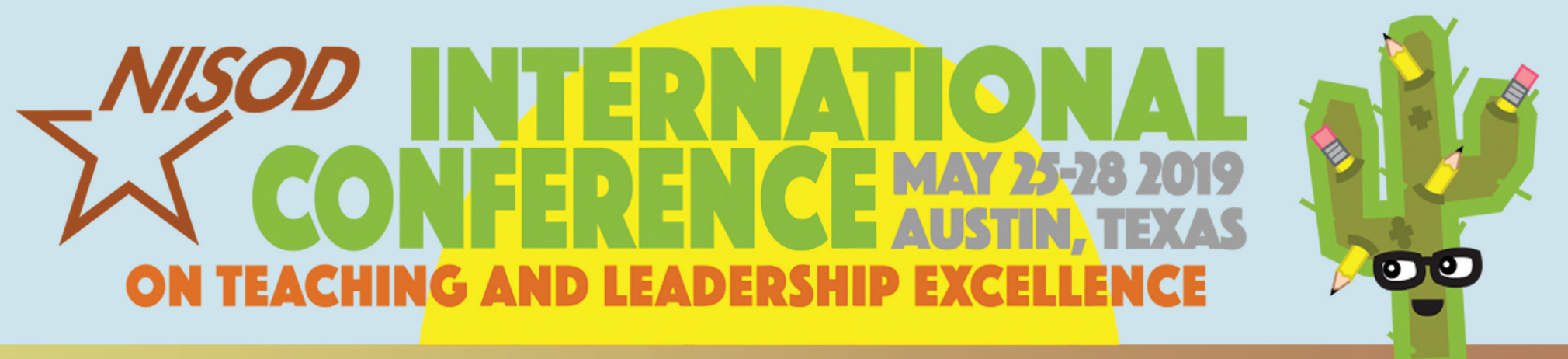

REGISTER TODAY AT NISOD.ORG/CONFERENCE

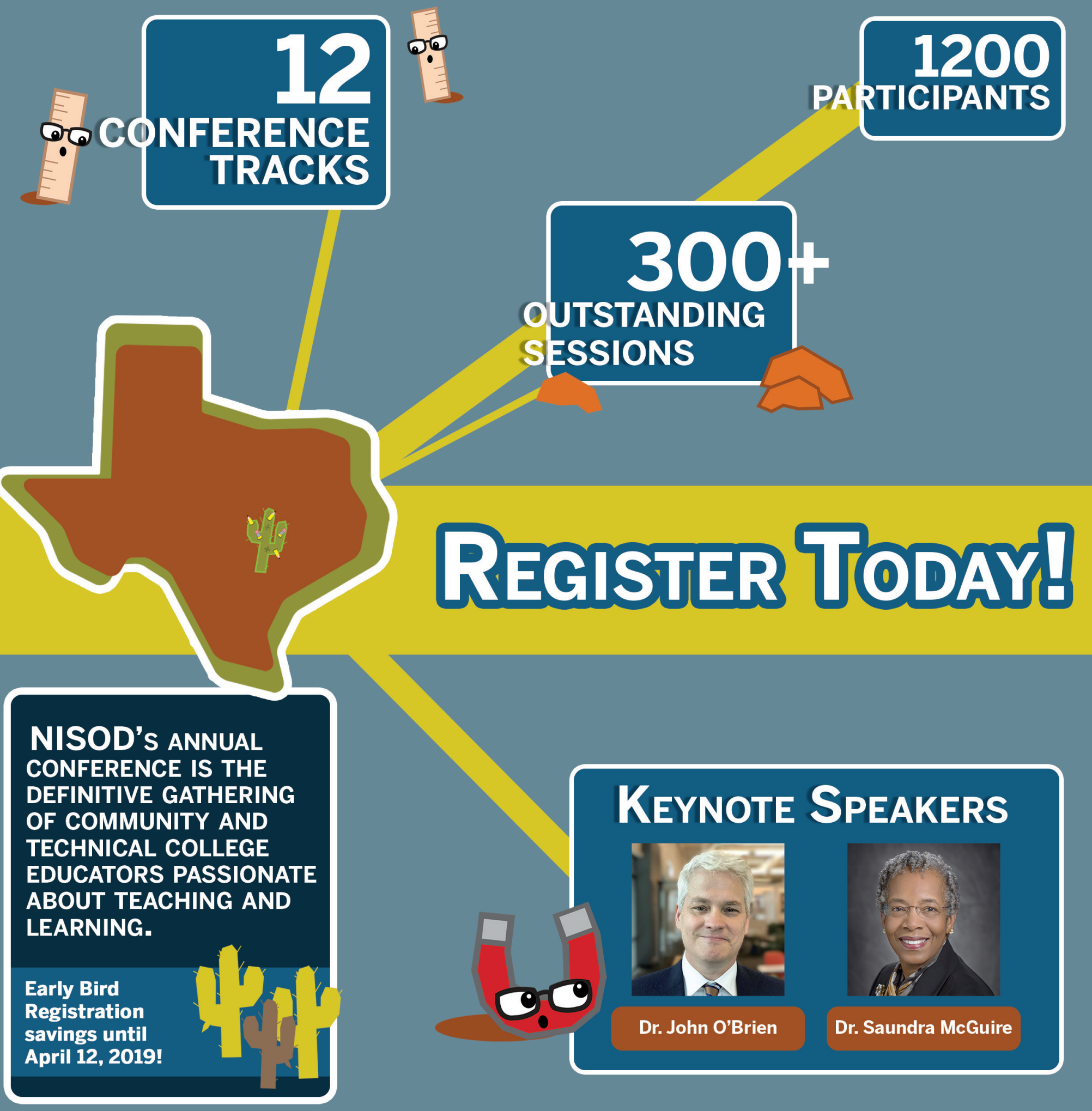


Broadening the definition of literacy and learn-

Exploring a Vision for a Global Literacy and Learning Environment: Addressing the Needs of Economically and Culturally Diverse Student Populations Through the Arts

\author{
Neva Cramer \\ Joan Bowman
}

\section{ABOUT THE AUTHORS}

Neva Cramer is currently the Director of Education and Graduate Studies in Education at Schreiner University. Neva's experience and academic background includes the performing arts, literacy and learning through the arts including training with Harvard's Project Zero Summer Institutes, and studying the effects of poverty on learning.

Joan Bowman is an Assistant Professor of Education at Schreiner University, where she prepares educators for leadership. Joan has served many years as assistant principal, principal, and associate superintendent. The districts where she served were 75 percent economically disadvantaged, which led to her deep concern for the effects poverty has on learning.

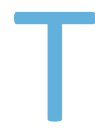

he learning process is just as much a cultural and social process as it is a cognitive process. To answer the call for diversity in the representation of knowledge for English language learners, we as educators must acknowledge and embrace a variety of means to express the learning experience. Words do not always transfer across cultures and experiential backgrounds, but communicating through the arts can open up doors for communication and learning for students who face the barriers of language or economic or cultural differences. The common limitation may be words as a second language. The global learning environment is the image, the multimedia, the music, the social media, and the web that many students from all backgrounds share. ing and thus our perspective on curriculum to include the visual and communicative arts can make school relevant across cultures and various backgrounds. Learning through the arts research and strategies can transform the twenty-first century classroom by making learning an experience that uses critical and creative thinking skills, communication, and collaboration to develop the intellectual character of students and enhance learning and literacy skills through an innovative learning environment designed for culturally and economically diverse students (Cramer, 2014; Jensen, 2013).

Using an arts-based literacy curriculum that integrates multiple ways of knowing can help students develop creative thinking skills and nourish their imaginations. Enhancing learning and literacy with the visual and communicative arts can provide a repertoire of scripts necessary for visualizing and negotiating meaning in the culturally diverse classroom of twenty-first century learners whose everyday literacy is collaborative and often image-based (Park, 2012).

The Effects of Poverty on Learning

Jensen (2013) presented a perfect case for using the arts and images for increasing vocabulary through his research on how poverty affects learning and engagement. Without an image, words can be meaningless. Words help students "represent, manipulate, and reframe information" (p. 25). Limited vocabulary can result in less comprehension. Less comprehension can result in less participation. Less participation can result in less learning.

\section{The Effects of the Arts as a Catalyst for Thinking and Learning}

Brooks (1999) advocated constructivist teaching practices that ask learners to transform and internalize information as opposed to the traditional college classroom, where information is just restated in different forms such as tests or reports. Instead, students should interact with information visually and through other forms of artistic expression. Then, students can shape their perceptions through the sharing of their perceptions with others

\section{Using an arts-based literacy curriculum that integrates} multiple ways of knowing can help students develop creative thinking skills and nourish their imaginations. 
whose experiences may provide insight by allowing the learner to see through the lens of experience, language, and culture of others (Jha, 2012, p. 171). As English language learners use language, images, and modern technologies to negotiate meaning and cross cultural and social understandings, learning is manifested in meaning construction - the process of forming a perception based on the imagery, form, and language of the visual or communicative arts text translated through the experience of the reader (Cramer, Ortlieb, \& Cheek, 2007).

There is much evidence from educational researchers in support of the arts as a catalyst for thinking and learning. In The Arts and the Creation of the Mind, Eisner (2002) validated the arts in education as a means of enhancing imagination and creative thought: "We do indeed see in our mind's eye" (p. 4). In further support of creativity and the communication and social skills necessary for learning and working, Eisner claimed that the arts help us create our lives by "expanding our consciousness, shaping our dispositions, satisfying our quest for meaning, establishing contact with others, and sharing a culture" (p. 4). Jensen (2001) reminded that it is not that students cannot learn without the arts, but that "the arts enhance the process of learning by nourishing the sensory, attentional, cognitive, emotional, and motor capabilities systems connected to learning" (p. 2).

\section{Benefits of Using the Arts as Pedagogy in the ELL Classroom}

According to the National Art Education Association (2018) - based in part on the introduction pertaining to the visual arts, dance, theatre, and music submitted to the Secretary of Education-proposed benefits of using the arts as pedagogy in the ELL classroom include the following:

1. Incorporating the arts to develop thinking dispositions can elevate current instruction modes to meaningful methods of learning that provide multiple ways of knowing and understanding across the various disciplines.

2. The arts can teach students to think by using multiple literacies to express themselves and communicate with others in a meaningful way through a common language-the image-especially for today's visual learners.

3. The arts can provide a means for multiple cultures to find common ground for understanding one another and our world. After all, symbols and images are the universal language.

4. The arts can allow students to appreciate and come to see the perspectives of others through a safe medium where there are always many ways to interpret, and they see through the lens of their experiences.

5. The arts can provide an aesthetic environment of learning, seeing, and creating where students' emotions, intuitions, and feelings matter, allowing them to learn with their whole being through their bodies, their voices, their minds, and their imaginations.
Communication Through the Arts Routine for ELL-an Exemplar for Success

In a previous publication (Cramer, 2014), I created a thinking and responding routine called The Four D's of Determining Meaning, which can be used to help develop oral and written communication skills for ELL students by initiating the lesson with a visual of the painting, Starry Night, by Van Gogh (see Figure 1). The Four D's of Determining Meaning are as follows:

1. Describe: First, students view and describe the image.

2. Discuss: In the second step, students discuss what they see with others to expand their vision and powers of observation.

3. Defend: In the third step, students defend their claims based on what they see, feel, or know. For example, one student defended his perception by noting the tiny village in the bottom of the picture where no one was seen outside, and the dark and a fire surrounded their village. He described the setting as a tiny village in Vietnam where the people of the village prayed to a shiny star for peace. His story ended with the star granting peace and happiness, and the people became "amicable." The student came from Vietnam and was able to view the image through the lens of his own experience using a digital translator to add the word amicable.

4. Direct: In the final step, students tell the story of the image in text by directing a performance of their understanding through a scripted narrative. For example, the students created a play where the villagers were fighting an enemy from another land. They wrote out the scene in dialogue including the plea to the shining star.

The Scene: A tiny village where people do not get along.

Villager 1: "We must flee this land and escape our enemy, or surely we will die."

Villager 2: "I am afraid and mad. Who will help us?" Then a shining star appears, and all people make a wish for peace.

Villagers: "Peace! Peace in our village!"

And people became amicable. The end.

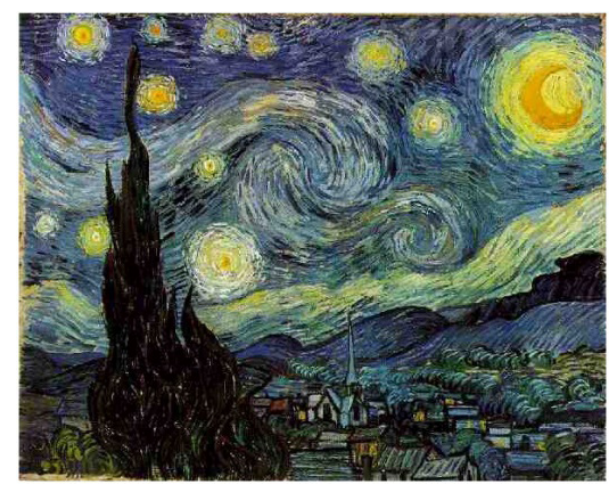

Figure 1: The image of The Starry Night by Vincent van Gogh is a faithful photographic reproduction of a two-dimensional, public domain, work of art. This file has been identified as being free of known restrictions under copyright law, including all related and neighboring rights. 


\section{Conclusion}

Many students from impoverished backgrounds or those struggling with cultural and language differences do not see themselves as thinkers and contributors to their world. Training educational leaders to use arts-integration strategies has the potential to transform our classrooms into cultures of inquiry that reflect critical and creative thinking and authentically prepare our students to be lifelong learners by incorporating socially interactive instruetion that promotes self-efficacy and develops effective college- and career-readiness thinking and communication skills in preparation for meeting the demands of career and life skills necessary for success in a global society (Cramer, 2018).

\section{References}

Brooks, J. G. (1999). In search of understanding: The case for constructivist classrooms. ASCD.

Cramer, N. (2014). Supporting literacy through the visual and communicative arts: Building momentum in literacy for 21st century digital learners. Texas Association for Literacy Education Yearbook: Building Momentum, 2, 62-77.

Cramer, N. V. (2018). Using trauma-informed pedagogy to make literacy and learning relevant and engaging for students of poverty. Texas Association for Literacy Education Yearbook: Building Momentum, 77.

Cramer, N., Ortlieb, E. T., \& Check Jr., E. H. (2007). Multiple ways of knowing: A theoretical framework for drama and literacy in a contemporary curriculum. Reading Matrix: An International Online Journal, 7(3), 35-43.

Eisner, E. W. (2002). The arts and the creation of mind. New Haven, CT: Yale University Press.

Jensen, E. (2001). Arts with the Brain in Mind. Alexandria, VA: ASCD.

Jensen, E. (2013). How poverty affects classroom engagement. Educational Leadership, 70(8), 24-30.

Jha, A. K. (2012). Epistemological and pedagogical concerns of constructionism: Relating to the educational practices. Creative Education, 3(2), 171-178.

Marzano, R. J. (2007). The Art and Science of Teaching: A Comprehensive Framework for Effective Instruction. Alexandria, VA: ASCD.

National Art Education Association. (2014). National core arts standards. Retrieved from www.nationalartsstandards.org

Park, J. Y. (2012). A different kind of reading instruction: Using visualizing to bridge reading comprehension and critical literacy. Journal of Adolescent \& Adult Literacy, 55, 629-640.

van Gogh, V. (1889). The starry night [Oil on canvas]. Museum of Modern Art, New York, NY. 
College Reading \& Learning Association (CRLA) strives

to be the professional development resource of choice

for college professionals dedicated to enhancing

student academic success. Its mission is to provide college reading and learning professionals with an

open forum to discover and exchange the leading tools and techniques to enhance student academic success.

Find out more about CRLA, its guiding principles, and membership benefits at www.crla.net

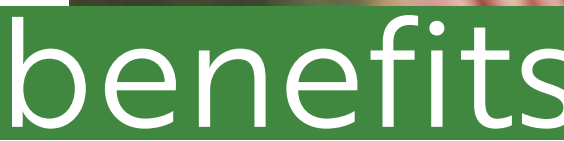

CERTIFICATION PROGRAMS: Create, improve, and expand tutor and mentor training for a campus' unique programs.

SPECIAL INTEREST GROUPS (SIG): Share and promote professional interests.

STATES, REGIONS, \& CHAPTERS (SRCs):

Connect with colleagues in the same geographic area.

AWARDS AND SCHOLARSHIPS: Recognize those who contribute significantly to the field and the organization.

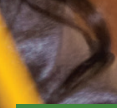

\section{a} tten

ANNUAL CONFERENCE: A schedule packed with pre-conference institutes, roundtables, concurrent sessions, as well as a variety of social and cultural events. Our 52nd Conference will be in New Orleans, October 30-November 2. Save the Date!

SUMMER INSTITUTE: Train the trainers in mentor and tutor training programs. Attendance is limited to provide opportunity to work in small groups and benefit from in-depth, personalized interaction with the instructors.

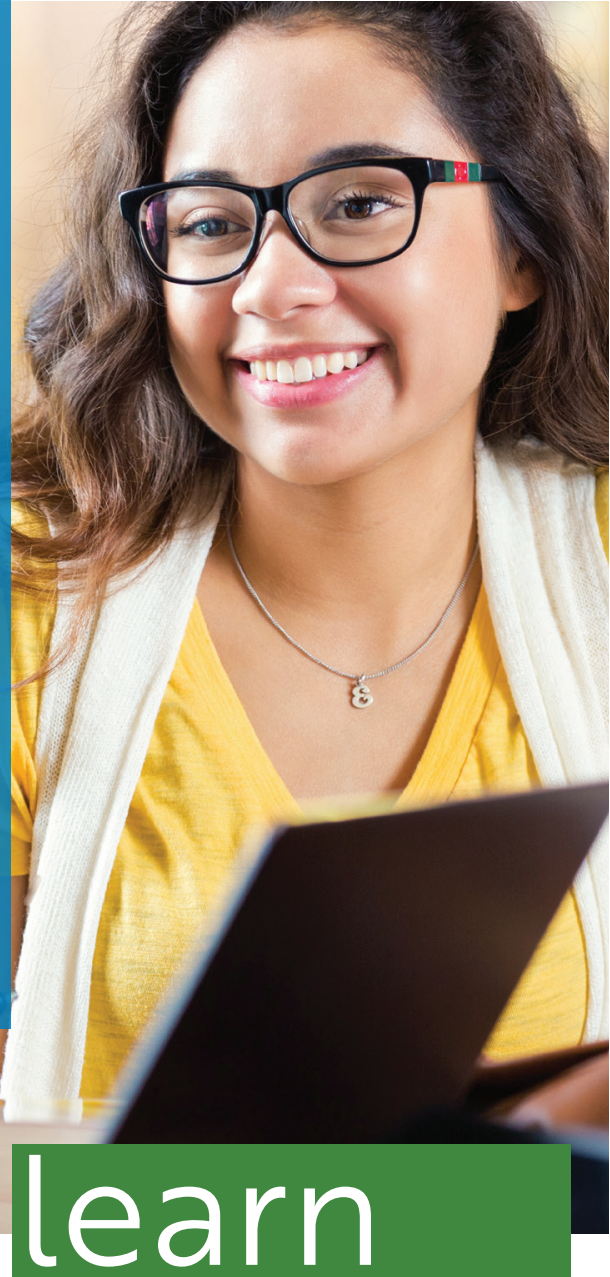

\section{JOURNAL OF COLLEGE READING} AND LEARNING (JCRL):

An international, peer-reviewed forum for theory, research, and policy related to learning assistance and developmental education at the postsecondary level.

WHITE PAPERS: Authoritative, evidence-based reports written by experts.

\section{Sharing the Best for Student Success!}

\section{JOIN TODAY! www.crla.net}

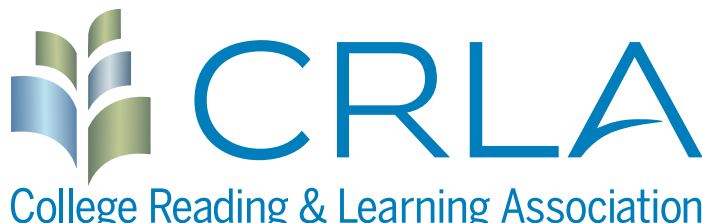

Proud Members of 


\section{UTILIZING GOOGLE MY MAPS IN THE CLASSROOM}

\author{
William S. Duffy
}

\section{ABOUT THE AUTHORS}

William S. Duffy is Faculty Fellow for Faculty Development at St. Philip's College. He received his Ph.D. in Classics from the University at Buffalo. He discovered Google My Maps as a teaching tool for his Ancient Travel and Ethnography Class and has since used it in several other courses.

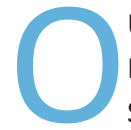

ur students are entering a world where they must both engage with maps and map-based software on a daily basis and communicate effectively through digital tools and media. Incorporating projects that allow students to build skills along these lines therefore has the potential to provide them with significant benefits. To that end, in Spring 2013, I introduced a Google My Maps project-" The Roadrunners' Guide to the Ancient World" (2019)into my Ancient Travel and Ethnography class. The Roadrunners' Guide was an interactive map with color-coded markers on a custom Google My Maps leading to travel-guide style descriptions of ancient cities, built by students in WordPress. This project led to increased student engagement, original and detailed projects, and a marked increase in student ownership of and pride in their work, which Conley and French (2013) among others see as key factors in student success. This experience, repeated in different courses and semesters, convinced me that Google My Maps provides teachers a versatile, valuable tool to help students build course specific knowledge and universally valuable skills with digital tools which, as Pfannenstiel (2010) notes, even modern students often struggle to use in academic and professional settings.

\section{Value to Students}

Integrating Google My Maps into the classroom can develop students' ability to utilize maps and special technologies. These skills are becoming increasingly vital to classroom and life success in all fields (Bednarz, Acheson, \& Bednarz, 2006). However, only $20 \%$ of $12^{\text {th }}$ graders scored at or above a proficient level in geography on the 2010 National Assessment of Educational Progress exam (the latest exam to measure these students in this field), the largest representative national assessment of student academic capacity (National Assessment of Educational Progress, 2010), lower than in the two previous examinations. This is the second lowest level of proficiency of all subjects tested, indicating that students have a significant deficiency in geographic knowledge that almost certainly follows them to college. Google My Maps-based activities therefore allow students to gain what they both lack and need.

Google My Maps-based projects also allow students to have what Lombardi (2007) describes as authentic learning experiences - using a software tool and digital skills they will use in their real and professional lives to create a resource that will be used outside the classroom. Education research suggests that students engaged in authentic learning have higher levels of perseverance, satisfaction, and success (Lombardi, 2007). In the context of authentic learning, Google My Maps' ability to allow users to change who can access or contribute to a given map at will provides an additional element of safety. Since teachers can choose when and how students can contribute to a given map project, and when and to what degree the final project will be made available to the public, they can ensure that student work is only presented to broader audiences when it is ready for the public. Students also have the ability to continue to improve (or remove) their work after the completion of their project, and share it with potentially interested parties. For example, in Spring 2013, two of my students presented their projects at national conferences, and one used his to win a post-graduation job.

\section{Designing Google My Maps Projects: Guidelines}

Google My Maps is a free and versatile tool that enables the creation of engaging class projects that help students learn skills that are vital in the modern age. However, as with all tools, faculty members should take care when designing a project around

\section{Google My Maps provides teachers} with a free and comparatively simple way to help students build their knowledge in geography, integrating with geographic software and digital publication while engaging deeply with course materials. 
the software. First, teachers should design a project that benefits from Google My Maps' geographic information and interactive interface. For example, while a heat map of access to health and child care might well be an excellent use of the Google My Maps interface, an analysis of universal best practices in childcare probably would not be. Second, teachers should give students (and themselves) access to an online Google My Maps tutorial and ample time to practice using it; teachers must not assume that students already have command over Google My Maps or any digital tool (Watson \& Pecchioni, 2011). Finally, teachers should take care when assessing student contributions to public maps, with a particular focus on plagiarism and acceptable discourse. If an individual student project is beneath the standards of the school, the teacher may have to request that the student redo the project before the class's creation is made public, or in extreme cases prevent public access to unacceptable student contributions. However, in my experience, such problems are extremely rare.

\section{Google My Maps Functions}

Google My Maps has four basic tools, each of which can be adapted for pedagogical purposes, either individually or in concert: layers, polygons, markers, and measurements. I have largely limited myself to markers in my student projects, although I plan to utilize more tools in the future. Below is a brief description of each tool and some of its potential educational functions:

- Layers create different maps that can be viewed or edited together or in isolation. This is useful for differentiating between different students work or if there is pedagogical value from overlaying different sets of geographic information (i.e. the borders of nations and empires in different eras).

- Polygons allow for the creation of shapes on the map. Once the polygon is completed, Google My Maps allows for the coloration and shading of the polygon and provides a marker in which one may include information or a link to outside media. This can be used to create heat maps and infographics that illustrate a wide variety of geographic and cultural information, such as the prevalence of food deserts in a given community.

- Markers place an icon on the map. Google provides a number of variant icons that can be used to signal information, and each icon has a space for providing additional information or links to outside resources. This is the most versatile Google My Maps tool for educational purposes. A Google My Maps resource can use the shapes and colors of the markers to provide information and/or organize the entries, and the entry space allows students to add written material, images, or external links to the resource. The information provided in these markers, and the markers Google My Maps automatically creates when a polygon or line is made, provides the easiest space for students to showcase their skills and course knowledge.

- The Measurement tool allows for the measuring of distance between points on the map. However, not all information accessed this way stays permanently on the site, so they may function better as parts of student assignments than as an element of a final, public Google My Maps project.

\section{Conclusion}

Google My Maps provides teachers with a free and comparatively simple way to help students build their knowledge in geography, integrating with geographic software and digital publication while engaging deeply with course materials. While adapting the tool to your course will take time and creativity, the potential rewards are more than worth the effort.

\section{References}

Bednarz, S. W., Acheson, G., \& Bednarz, R. S. (2006). Maps and map learning in social studies. Social Education, 70(7), 398-404.

Conley, D. T., \& French, E. M. (2013). Student ownership of learning as a key component of college readiness. American Behavioral Scientist, 58(8), 1018-1034.

Lombardi, M. M. (2007). Authentic learning for the 21st century: An overview (ELI Paper 1: 2007). Retrieved from Educause website: https://library.educause.edu/-/media/files/ library/2007/1/eli3009-pdf.pdf

Pfannenstiel, A. N. (2010). Digital literacies and academic integrity. International Journal for Educational Integrity, 6(2), 41-49.

National Assessment of Educational Progress (2010). The nation's report card: Geography grade 12 national results. Retrieved from https://www.nationsreportcard.gov/ geography_2010/g12_nat.aspx

The roadrunners' guide to the ancient world. (2019, May 29). Retrieved from roadrunnersguidetotheancientworld.com/

Watson, J. A., \& Pecchioni, L. L. (2011). Digital natives and digital media in the college classroom: Assignment design and impacts on student learning. Educational Media International, 48(4), 307-320. 


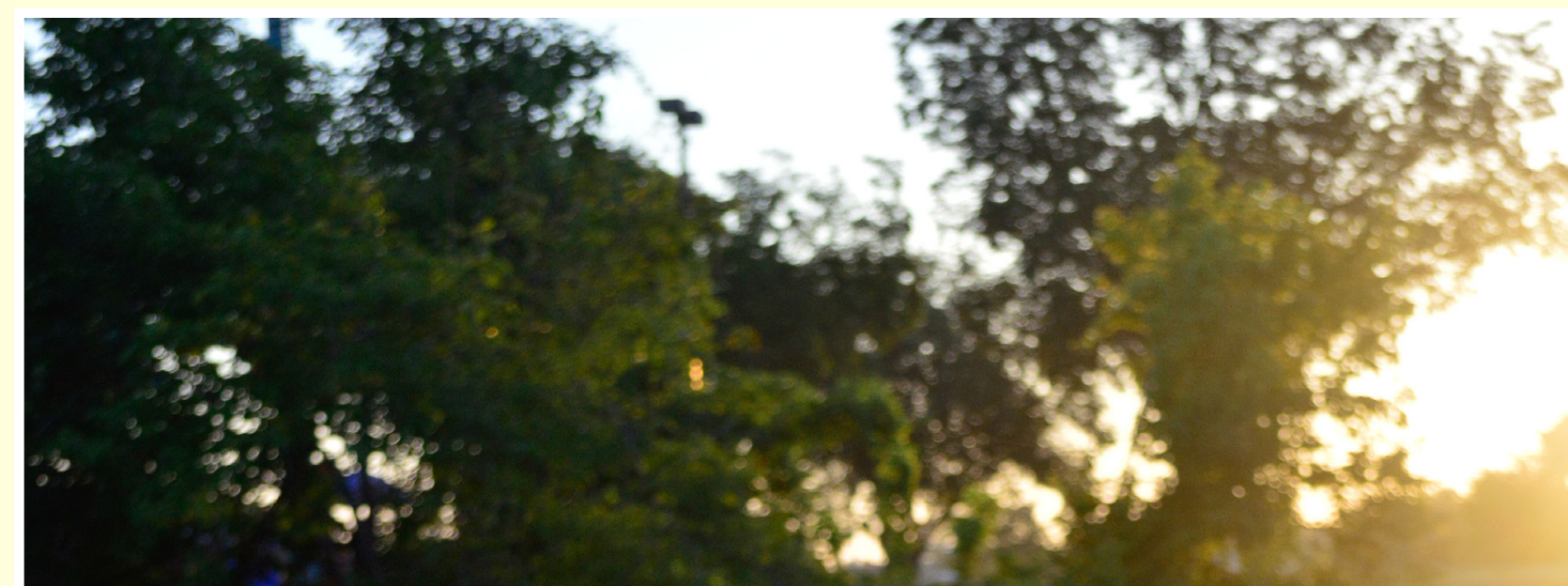


Learning Alone and Academic Capital: An Old Sociological Idea in a New Educational Application

\section{Jack Trammell}

\section{ABOUT THE AUTHOR}

Jack Trammell is chair of the Sociology, Criminal Justice, and Human Services Department at Mount St. Mary's University in Maryland. He specializes in social history, disability, education, and human services.

\section{T} he concept of social capital has been discussed for more than 75 years, pioneered by social scientists such as Bourdieu's (1996) theory of capital, Coleman's (1990) rational choice theory, and Putnam's (2000) democratic theory. Despite a great deal of debate about specific definitions, social capital can generally be defined as the transactional value of relationships, networks, associations, and social knowledge-in essence, a commodity that people can earn, store, spend, and invest toward specific purposes and goals.

Academic capital, a relatively new concept, can be seen as a subset within the larger world of social capital and/or cultural capital (Trammell, 2018a; Trammell, 2018b). In this framework, academic capital is defined in similar general terms: the transactional value of relationships, networks, associations and social knowledge, only geared specifically toward situations and environments particular to education (and in this conversation, higher education). The development of new instruments, such as the Combined Measures of Academic Capital Survey (CMACS), is part of the developing concept (Trammell, 2019).
Utilizing the concept of academic capital allows for a number of useful innovations, particularly in areas like developmental education, universal design in instruction, and learning assistance, where researchers have struggled to combine or reconcile awkward combinations of theoretical paradigms (e.g. the overlap between critical race theory and ESL). Students in this new theoretical framework can be gifted cognitively and successful in secondary experiences yet still come to college with academic capital assets that vary widely. Academic capital provides a new way to assess how "at-risk" a student is based on measures more separable from race, socio-economic status, disability, or other traditional minority identity statuses that involve complicated historical stigmas.

As a theory, academic capital has the promise of transforming learning assistance and higher education in several ways, but one perhaps more important than the others is the possibility of moving beyond deficit models that frame developing students as needing something. Instead, academic capital recognizes that everyone is their own agent with control over where they want to spend, invest, or develop their own academic capital. Just as Wall Street is incredibly diverse, with a sometimes-overwhelming collection of tools and possibilities, so academic capital opens a rich window on educational success that does not rely on simply reading better or getting accommodations. This theory may seem to be
too good to be true, although con-

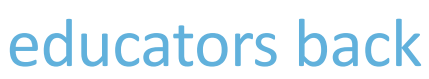

to the primary

importance of relationships,

which is what the

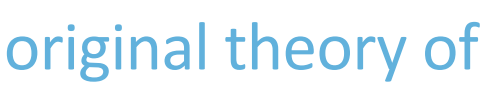

social capital was all about. sidering some basic initiatives that could be part of this shift is not overwhelming. Some of these ideas include the following: a dramatic reframing of new-student orientation away from simply how to get help and more toward developing relationships that will matter-when you develop your academic capital you know where to get help; encouraging living and learning communities (LLCS) where faculty, staff, and students interact together, creating and banking academic capital; and continuing to reframe the college/ university experience as having fuzzy edges (i.e. doesn't really begin or end in the strictly dichotomous sense). There are many other ideas and possibilities, including some with direct implications for tutoring, ESL, and disability programs.

Putnam's original sociological concept of social capital lamented the trend that modern society 
seemed to indicate that people were becoming more distant from each other (his example was bowling leagues) (Putnam, 1995). He pointed out that more people bowl now than in decades past, and yet bowling leagues (i.e., social networks related to bowling) have nearly disappeared. In the same way, areas of the academy have often become encased in silos and students can be overwhelmed, particularly at larger institutions. Academic capital is a framework that takes educators back to the primary importance of relationships, which is what the original theory of social capital was all about.

\section{References}

Bourdieu, P. (1986). The forms of capital. In J. Richardson (Ed.), Handbook of Theory and Research for the Sociology of Education (pp. 241-258). New York, NY: Greenwood Press.

Coleman, James S. (1990). Foundations of Social Theory. Cambridge, MA: Harvard University Press.

Putnam, R. D. (1995). Bowling alone: America's declining social capital. Journal of Democracy, 6(1), 65-78. https://doi.org/10.1353/jod.1995.0002

Putnam, R. D. (2000). Bowling alone: The collapse and revival of American community. New York, NY: Simon \& Schuster.

Trammell, J. (2018a). Learning alone: Academic capital and learning assistance. Paper presented at the annual conference of the College Reading and Learning Association. Albuquerque, NM.

Trammell, J. (2018b). Learning alone: Academic capital and learning assistance. Paper presented at the annual conference of the National College Learning Center Association. Niagara Falls, NY.

Trammell, J. (2019). Combined measures of academic capital survey (CMACS). In progress. 
FOR

\section{ADULT AND DEVELOPMENTAL EDUCATORS AND LEARNING SKILLS SPECIALISTS July 13-26, 2019}

Further your knowledge in the theory and best practices of postsecondary developmental education and learning assistance. Institute seminars combine expert-led presentations with learning activities that explore practical applications to promote student persistence and retention. During the summer residency, a dynamic living-learning community provides opportunities for sharing ideas. Networking among educators representing a variety of cultural and ethnic backgrounds is a key benefit. Kellogg attendees develop an action plan to address issues affecting their own institutions' programs. Those interested in Developmental Education Specialist Certification enroll in an optional 3-credit-hour graduate course and design a campus innovation project to implement in the academic year following the residency.

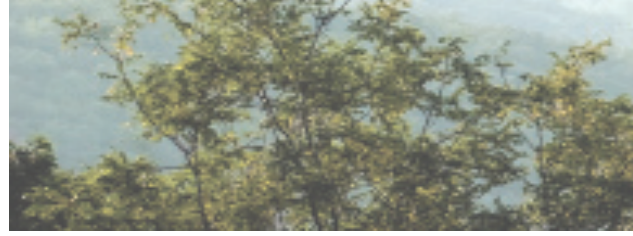

AdvancedlKelloggi]nily28-August2,2019

FEATURED TOPICS:

- Implementing innovative models for retention \& completion

- Connecting courses and support services

- Applying technology for student success

- Understanding the characteristics of diverse learners

\section{"Positively Addressing the Reform Mlovement"}

For Information or to Apply:

Kellogg Institute - ASU Box 32098

Boone, NC 28608

kellogg@appstate.edu

www.ncde.appstate.edu/kellogg

The premiere book release from the

Council of Learning Assistance and Developmental Education Associations is

\section{NOW AVAILABLE!}

Co-published with the National Center for Developmental Education's DevEd Press, The Profession and Practice of Learning Assistance and Developmental Education: Essays in Memory of Dr. Martha Maxwell can be used for scholarly research, professional development enhancement, course text material, and applications to practice. The book is first of its kind, honoring Martha Maxwell's vision and featuring chapters from the CLADEA Fellows; it is organized into sections by historical context, current issues, and best practices.

"I invite readers to enjoy this unique collection of wisdom, research, practice and advice-all written to honor and pay tribute to Martha Maxwell, one of the profession's greatest treasures." -Russ Hodges, CLADEA Chair 2007-2014

\section{Order your copy today from}

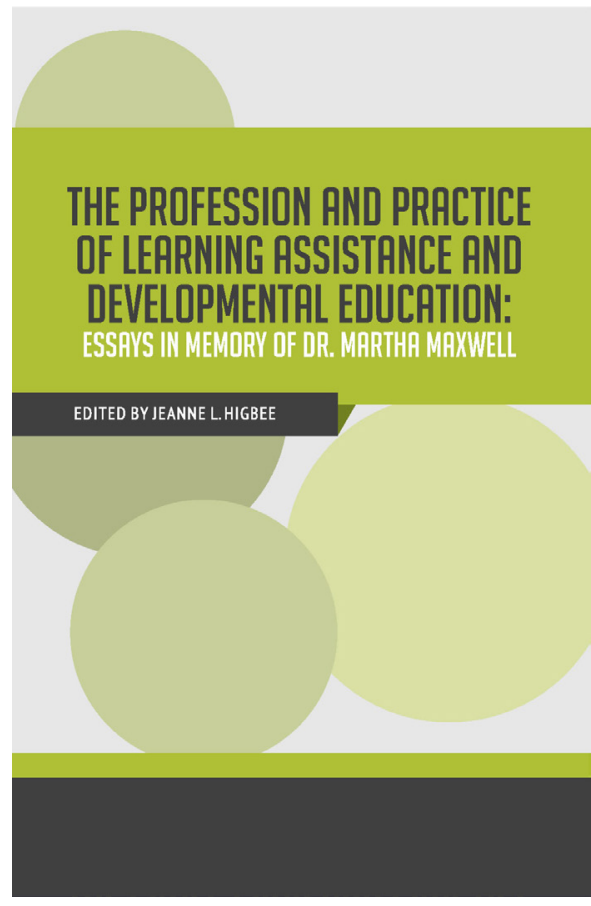

\section{\$35 each includes U.S. shipping}
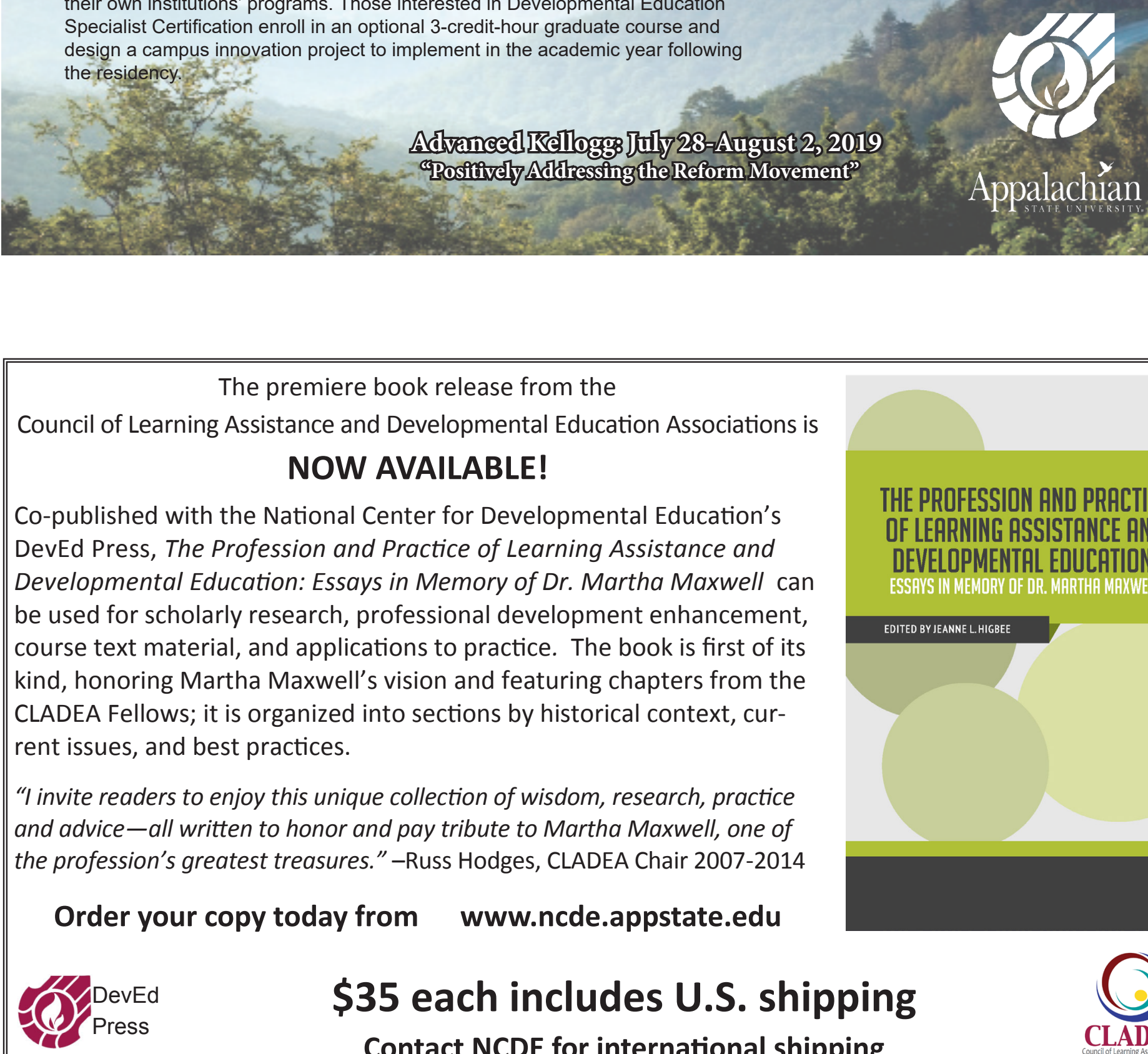

Contact NCDE for international shipping 


\section{Building the Discipline- Specific Classroom: A Pedagogical Discussion}

\section{Leta Deithloff}

\section{ABOUT THE AUTHOR}

Leta Deithloff, Ph.D., has been teaching and designing integrated reading/writing since 2008 and the newly-mandated corequiste courses since 2015. A life-long learner, advocate for wholistic student development, and recipient of the University of Texas at Austin's coveted Hairston Prize for Excellence in Teaching, "Dr. D" welcomes information on quality activities for the classroom.

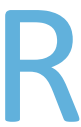

ecent discussions with colleagues about student progress and well-being seemingly lead to discipline-specific reading concerns. Further cross-campus conversations reveal that the concern about how students can best manage heavy reading loads in often unfamiliar subjects transcends developmental education as seasoned and successfully-transitioned students find themselves unprepared for upper-level reading demands. Faculty representing different departments and varied teaching levels have a request for those in student support roles: How best can I help my students?

Yet, this conversation is not new. In 2008, Shanahan and Shanahan implicated an increasing need for literacy, particularly higher-level literacy skills, because assessment data revealed that today's adolescents had not improved and were perhaps worse readers than the previous generation. The authors' conclusions for addressing this need included direct guidance for learners in meeting particular disciplinary reading and writing demands. So how can educators best serve student disciplinary literacy needs now?

Fortunately, some key recommendations offer a nice curriculum-development starting point. Duke and Pearson (2002) indicated that effective comprehension instruction should be balanced between explicit comprehension instruction strategies and extensive time and opportunity for textual practice, production, and discussion. Graham and Hebert (2010) confirmed that successful classrooms should use integrated methods: "writing practices complement reading practices and should always be used in conjunction, with each type of practice supporting and strengthening the other" (p. 29). Furthermore, in a recent analysis of common practices for content-area instruction, Gabriel and Wenz (2017) suggested that, although educators differ on how to teach disciplinary literacy, most agree that effective instruction views learning as an apprenticeship into communities with agreed-upon conventions that guide the production, dispensation, and evaluation of disciplinary knowledge. Thus, the central goal of disciplinary literacy instruction becomes to help learners achieve insider access in these communities so that learners are active rather than passive observers.

Given these guidelines, educators can construct a course basis that builds upon what is known and adopts a blended approach from Gabriel and Wenz's (2017) identified practices-discipline-specific strategy instruction using complex disciplinary texts, general strategy instruction to enhance foundational skills that then fit content-area reading and writing tasks, and engagement in the discipline that immerses students in the act of creating content-area texts by doing the specified discipline. Why address all three practices? Because one may not be enough. For students to be successful, courses should avoid teaching strategies, even content-specific ones, in isolation, or risk teaching learners skills they have difficulty generalizing. Instruction should be fluid across the literacy that students will need rather than just the skills educators think students should have. After all, the ultimate goal is for students to invoke their own learning solutions to the different problems they will encounter, so transferability is crucial. Students then confirm newly acquired knowledge by producing work within the demands of a certain discipline, making practice and modeling essential. Additionally, after ten years of classroom experience as an instructor, I notice that students often struggle with inferencing-or reading between the lines-regardless of discipline, so insight into decoding and understanding inferences would benefit generalized student comprehension.

Keeping in mind this conceptual base that focuses on disciplinary literacy as well as inferencing and textual decoding strategies, The University of Texas will pilot a course this summer that attempts to address both faculty concerns and student needs. The course will offer 
a blend of short stories based on student interest and selected chapters from the differing disciplines the stories invoke. This proposed course allows students to engage with texts, adapt inferencing strategies, and continue practicing literary analysis as they learn the underlying premise of various disciplines. As an example, students would start with the excerpt from Adams's The Hitchhiker's Guide to the Galaxy (1980) in which two computer programmers question the computer they designed, Deep Thought, which is "the second greatest computer in the Universe of Time and Space" (p. 112), about the answer to life, the universe and everything. The satirical response pokes fun at philosophical texts while it entertains. For the humorous argument to be successful, however, students must understand the basic message, purpose, and structure of typical philosophical readings. Thus, students would then read chapters from Nagel's (1987) philosophy text What Does It All Mean? Additionally, they will read How to Think Like a Computer Scientist (Wentworth, Elkner, Downey, \& Meyers, 2018) since Adams also satirizes computer programmers. Students will learn strategies for approaching these disciplines, read the texts accordingly, and then comparatively analyze the short story to identify the basis for the humor and ultimately evaluate its effectiveness.

This proposed course represents just one of many possible proposed courses for addressing discipline-specific literacy curriculum needs. Its premise is based on student interest because part of the challenge of becoming a good reader is feigning interest when texts are uninteresting, or more appropriately, too unfamiliar to be interesting. Thus, an important goal of instruction is teaching students to become engaged enough to comprehend challenging texts. But again, this is just one proposed course, the results of which will be revealed after the pilot.

Until then, let this exploration begin a discussion. What does effective discipline-specific curriculum look like?
References

Adams, D. (1980). The Hitchhiker's Guide to the Galaxy. New York: Harmony Books, 111-120.

Duke, N. K., \& Pearson, P. D. (2002). Effective practices for developing reading comprehension. In A. E. Farstrup \& S. J. Samuels (Eds.), What research has to say about reading instruction (3rd ed., pp. 205-242). Newark, DE: International Reading Association.

Gabriel, R., \& Wenz, C. (2017). Three directions for disciplinary literacy. Educational Leadership, 74(5). Retrieved from http://www.ascd.org/ publications/educational-leadership/feb17/ vol74/num05/Three-Directions-for-DisciplinaryLiteracy.aspx

Graham, S., \& Hebert, M. (2010). Writing to read: Evidence for how writing can improve reading. Carnegie Corporation Time to Act Report. Washington, DC: Alliance for Excellent Education. Retrieved from https://www.carnegie.org/media/filer_ public/9d/e2/9de20604-a055-42da-bc0077da949b29d7/ccny_report_2010_writing.pdf

Nagel, T. (1987). What does it all mean?: A very short introduction to philosophy. New York, NY: Oxford University Press.

Shanahan, T., \& Shanahan, C. (2008). Teaching disciplinary literacy to adolescents: Rethinking content area literacy. Harvard Education Review, 78, 40-59.

Wentworth, P., Elkner, J., Downey, A., \& Meyers, C. (2018). How to think like a computer scientist: Learning with Python 3 documentation release (3rd ed.). Retrieved from https://buildmedia. readthedocs.org/media/pdf/howtothink/latest/ howtothink.pdf 


\section{Developmental Education Administration}

Doctorate of Education

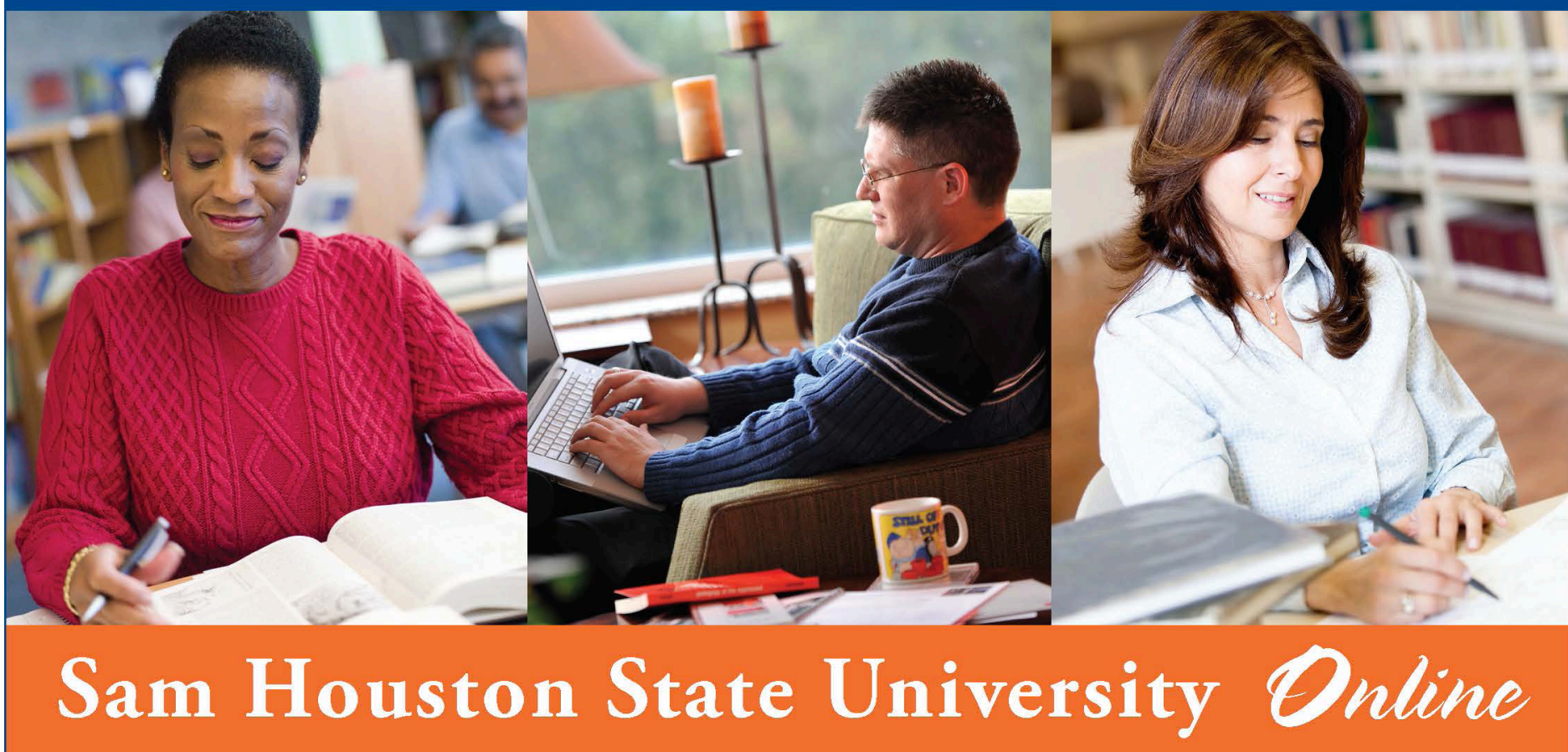

Preparing professionals to design and lead programs that help students succeed.

- $100 \%$ online program

- Flexible course scheduling

- Designed for working professionals

- Scholarships available

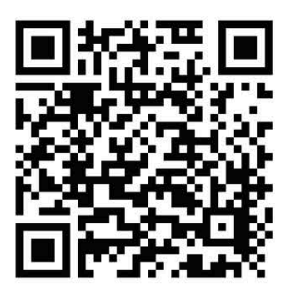

March 1 Application Deadline New Cohorts Begin Each Summer Apply Today at www.ApplyTexas.org
For more information, please contact:

D. Patrick Saxon

patrick.saxon@shsu.edu 936.294.1147

Or visit the program website at: http://bit.ly/SHDevEd

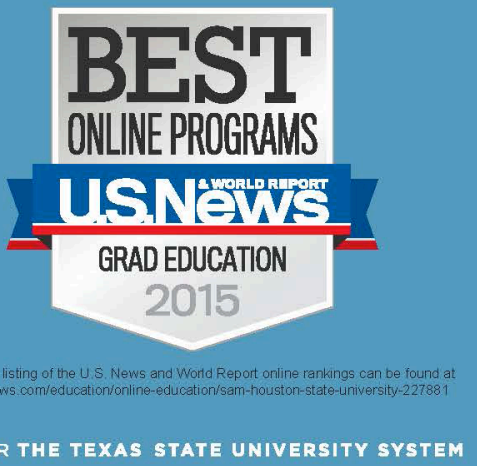


Although I wouldn't refer to gatekeeper and/or

\section{Freshmen, Teaching, and Tenure: Why Do We Call It the Trenches, Anyway?}

\section{Ann-Marie Lopez}

\section{ABOUT THE AUTHOR}

Ann-Marie Lopez is a professor of English at McMurry University in Abilene, Texas. She received her Ph.D. in English (Written Discourse: Theory and Practice) from Texas A\&M University-Commerce in 2013. A few of her research interests include: basic writing pedagogy; composition and student-athletes; literacy studies; rhetoric of young mothers/ parents; and disability and embodied rhetoric.

am a professor at a small, private liberal arts institution, and every semester I hear the same sentiment from my colleagues: "Three sections of freshmen writing?! I feel for you being in the trenches like that."

Trenches. The word sticks with me, and I wonder when this term-one that carries with it such negative connotations-became a euphemism for teaching first year students. A "trench," according to most dictionaries, is a ditch dug by soldiers seeking refuge from enemy attack. So, is teaching first year classes akin to being in the dirt, hiding from gunfire, scared to death? The metaphor is, I think, a bit problematic. Did the moniker emerge because of the work involved with teaching those classes (resulting in less time for scholarship/upper level courses)? Or, is it about the students in the courses (likely non-majors or students in developmental education courses)? Perhaps it is a combination, but a common view is that professors, particularly tenured ones, should avoid the trenches to ensure their own academic safety by teaching upper-level classes. Tenured or not, however, academic safety is invariably tied to institutional safety, right? And don't we as teachers need students in order to do what we as teachers do?

developmental courses as the trenches, students may perceive these classes as such. To be sure, these classes may be places where first-year and/or non-traditional students may have to fight to learn, particularly if labeled developmental. Many of my students never wrote an essay or cracked a book in high school. Most were told at least once in their years of schooling that they "can't write." Tenured faculty often lament these details, poring over the many ways in which these students are unprepared for college. But, what's wrong with tenured faculty teaching these classes, hoping to make a difference in not only retention but also students' overall classroom experience? Idealistic? Perhaps.

Here's the thing: We openly discuss retention and emphasize university-wide endeavors to keep students, but the one idea we usually don't consider is how tenured or full-time faculty teaching first-year courses could be a simple solution. The first year is, no doubt, the most crucial in regard to retention, and we know that less-experienced instructors-especially adjunct instructors whose connection to the institution can be fleeting, tenuous, and contingent-very often are relegated to teach introductory, foundational, developmental, and gateway courses. (Supiano, 2018). If this is the case, then aside from financial factors such as institutional budgets and full-time salaries, why are these courses often taught by part-time faculty with little to no support from or ties to the institution? and emphasize university-wide endeavors to keep students, but the one idea we usually don't consider is how tenured or fulltime faculty teaching first-year courses could be a simple solution.
Research indicates (Supiano, 2018; Jenkins, 2015; Umbach, 2007) that adjuncts are typically not as engaged in the institution or provided with the necessary tools to effectively support students in their classes. I am not suggesting these instructors are not qualified or shouldn't teach; however, I am suggesting that, given what we as professionals in higher education know about adjuncts teaching these courses-foundational, gateway ones-why do we continue to delegate them thusly? This, too, can be problematic considering that the quality of students' classroom experience seems to be congruent with their consequent persistence and success (Jenkins, 2015).

That said, if encouraging tenured faculty to teach these classes could make a difference in retaining at least one more student, then perhaps we should all be more proactive in such an endeavor. If more full-time faculty and administrators were, then maybe we wouldn't need to be concerned about anyone being in or out of the trenches. And, to the tenured 
faculty still on the fence, I offer this: If the fear is that students won't perform to your standards, remember that, typically, "Students will float to the mark you set" (Rose, 2005, p. 26). Set it high and meet them on the summit to address the challenges they may encounter rather than lament about or wallow in the trenches.

\section{References}

Jenkins, R. (2015, May 18). Retention in the trenches: What can you do in your own classroom to keep students on track? The Chronicle of Higher Education. Retrieved from https:// www.chronicle.com/article/Retention-in-theTrenches/23021

Rose, M. (2005). Lives on the boundary: A moving account of the struggles and achievements of America's educationally underprepared. New York, NY: Penguin Books.

Supiano, B. (2018, April 15). It matters a lot who teaches introductory courses. Here's why. The Chronicle of Higher Education. Retrieved from https://www.chronicle.com/article/It-Mattersa-Lot-Who-Teaches/243125

Umbach, P. D. (2007). How effective are they? Exploring the impact of contingent faculty on undergraduate education. The Review of Higher Education: Journal of the Association for the Study of Higher Education, 30(2), 91123. doi: $10.1353 /$ rhe. 2006.0080 


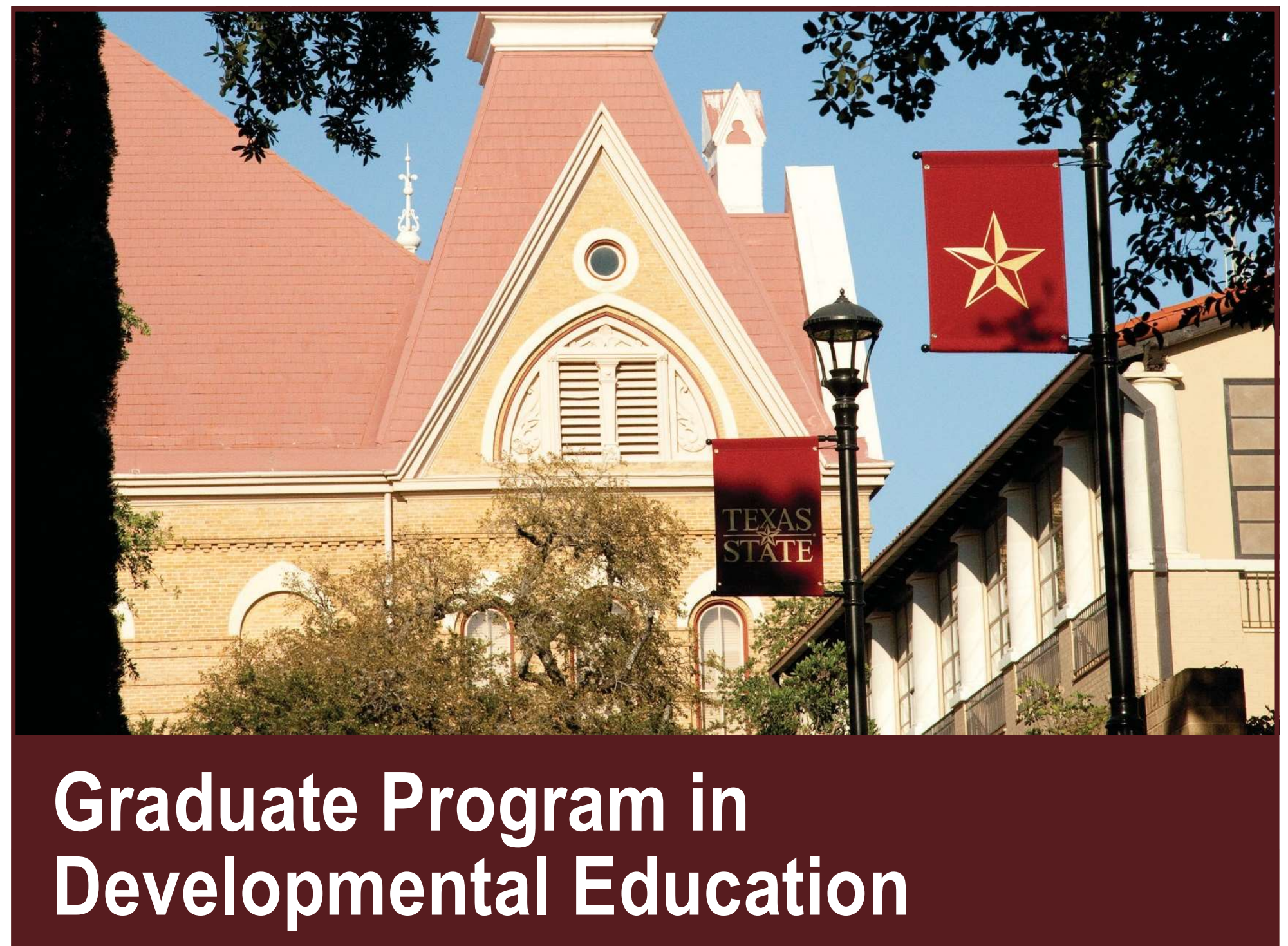

With degree concentrations in Learning Support, Developmental Mathematics, and Developmental Literacy

Ph.D. in Developmental Education

Ed.D. in Developmental Education

M.A. in Developmental Education

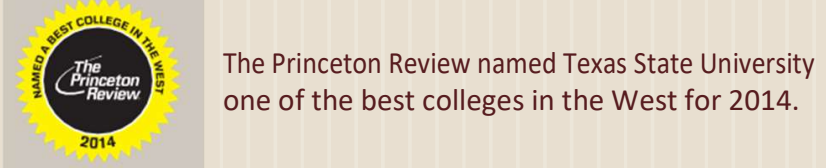

For more information, call 512.245.6839

facebook.com/DevEdTxSt @DevEdTxSt

Texas State University is an equal opportunity educational institution. education.txstate.edu/ci/dev-ed

\section{Faculty}

Taylor Acee, Ph.D.

Sonya L. Armstrong, Ed.D.

David C. Caverly, Ph.D.

Carlton J. Fong, Ph.D.

Russ Hodges, Ed.D

Jodi Patrick Holschuh, Ph.D.

Rebecca Jackson, Ph.D.

Eric J. Paulson, Ph.D.

Emily Miller Payne, Ed.D.

Emily Suh, Ph.D.

Emily J. Summers, Ed.D.

Alexander White, Ph.D.

$$
\begin{aligned}
& \text { TEXAS STATE } \\
& \text { UNIVERSITY } \\
& \text { The rising STAR of Texas }
\end{aligned}
$$




\section{Journal of College Academic Support Programs Call for Submissions August 1, 2019}

Supported by the Texas Association for Developmental Education (TADE), the Texas Chapter of the College Reading and Learning Association (TxCRLA), and the Texas State University's Graduate Program in Developmental Education, the Journal of College Academic Support Programs (J-CASP)—a double-masked, peer-reviewed, biannual scholarly journal relevant to Texas developmental education and learning assistance professionals-seeks feature articles and promising practices for the Fall 2019 issue.

For double-masked peer review, the J-CASP seeks scholarly research articles, research-to-practice articles, theory-to-practice articles, and reviews of literature that include recommendations and implications.

Appropriate articles that are not write-ups of empirical research studies will be considered for double-masked peer review based on scholarly rigor. More reflective, practitioner-based articles and op-ed articles or opinion pieces will be considered for publication as non-peer-reviewed promising practices.

The J-CASP is now accepting exploratory pieces. These short articles should be approximately 500 words with at least 2-3 references cited in the text. Exploratory pieces should introduce a conversation and generate implications for future research and practice. Whereas a promising practice can be considered a stepping stone toward further scholarly rigor, an exploratory piece can be considered as opening the door for further inquiry.

Appropriate manuscripts will undergo a peer-review process by members of the J-CASP editorial board. The review process will take approximately six weeks, including two weeks for authors to address reviewer comments. Submit your manuscript as a Microsoft Word (.doc, .docx, etc.) file, double-spaced with 12-point Times New Roman font. Your manuscript should not exceed 6,000 words (contact editor if such is the case) and must adhere to the APA Publication Manual ( $6^{\text {th }}$ edition) guidelines for writing, citation, and documentation style. Please include an abstract not exceeding 250 words.

Promising practices articles should not exceed 1,000 words (contact editor if such is the case) with at least 312 APA references cited in the essay. For questions, comments, concerns, or suggestions as well as to submit promising practices articles, please use the following email address: JCASP_Editor@txstate.edu.

Please refer to the J-CASP website for full submission information and to view current and past issues at https://journals.tdl.org/jcasp

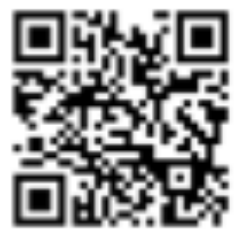

